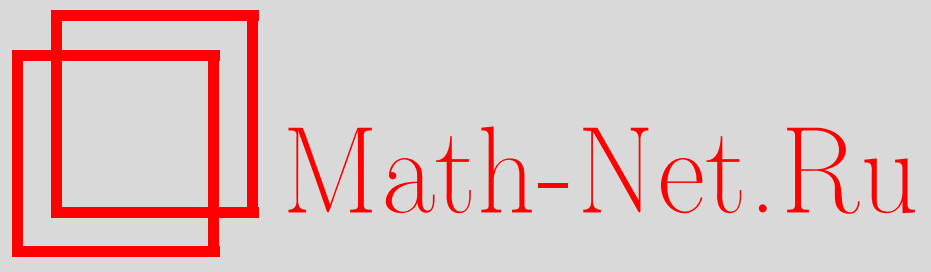

В. Драгович, М. Раднович, Интегрируемые биллиарды и квадрики, УМH, 2010, том 65, выпуск 2, 133-194

DOI: https://doi.org/10.4213/rm9349

Использование Общероссийского математического портала Math-Net.Ru подразумевает, что вы прочитали и согласны с пользовательским соглашением http://www . mathnet.ru/rus/agreement

Параметры загрузки:

IP: 54.197 .217 .227

26 апреля 2023 г., 15:25:30

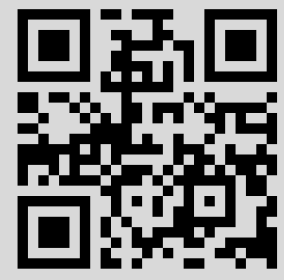




\section{Интегрируемые биллиарды и квадрики}

\section{В. Драгович, М. Раднович}

Изучаются биллиарды внутри квадрик как динамические системы с богатой геометрической структурой. Связи между биллиардной динамикой и геометрией пучков квадрик исследуются в обоих направлениях. Несколько хорошо известных классических и современных результатов, относящихся к роду 1 , обобщаются на произвольные размерность и род. Среди них теоремы Понселе, Дарбу, Вейра, пространственная теорема Гриффитса-Харриса. Излагается синтетический подход к теоремам сложения для рода большего 1.

Библиография: 77 названий.

Ключевые слова: интегрируемые биллиарды, пучок квадрик, гиперэллиптическая кривая, якобиан, поризм Понселе, периодические траектории, сетки Понселе-Дарбу, теоремы сложения.

\section{СОДЕРЖАНИЕ}

1. Введение........................................... 134

2. Биллиарды в эллипсоидах и их периодические траектории . . . . . . . 137

2.1. Периодические траектории внутри $k$ софокусных квадрик в евклидовом пространстве......................... 138

2.2. Биллиарды в эллипсоидах как системы с дискретным временем 145

2.3. Теорема Понселе и условие Кэли в пространстве Лобачевского . 150

2.4. Топологические свойства эллиптического биллиарда ......... 152

2.5. Интегрируемые возмущения потенциалом для эллиптического

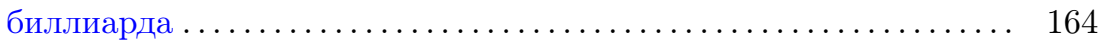

3. Закон биллиардного отражения и гиперэллиптические кривые........ 168

3.1. Динамика биллиардов в проективной постановке............. 168

3.2. Обобщенная кривая Кэли ................................ 171

3.3. Закон биллиардного отражения и алгебраическая структура на

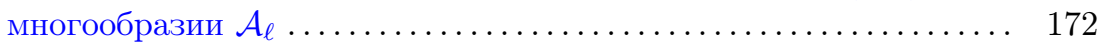

Работа выполнена при поддержке Сербского министерства науки и техники (проект "Геометрия и топология многообразий и интегрируемые динамические системы") и исследовательской группы по математической физике университета Лиссабона (проект "Вероятностный подход к конечномерным и бесконечномерным динамическим системам", PTDC/MAT/104173/2008).

(C) В. ДРАГович, М. РАДНович, 2010 
3.4. s-слабые траектории Понселе .........................

3.5. Обобщенная теорема Вейра и пространственная теорема Понселе в форме Гриффитса-Харриса в больших размерностях .....

3.6. Сетка Понселе-Дарбу и многомерные обобщения ............ 187

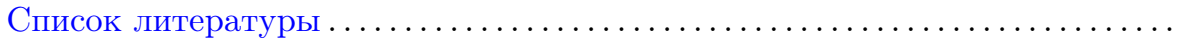

\section{1. Введение}

Двадцать лет назад В. В. Козлов и Д. В. Трещев дали в [1] хорошее обстоятельное изложение теории биллиардов, представив ее как раздел теории столкновений и динамических систем с односторонними связями. Они изложили различные аспекты динамических и аналитических свойств биллиардных систем. В частности, глава 4 книги посвящена интегрируемым задачам. Основная цель настоящей работы - обзор результатов по интегрируемым биллиардам, полученных за последние 20 лет. Мы начнем с биллиардов в областях, ограниченных коническими сечениями, и перейдем к их естественным многомерным обобщениям - биллиардам в областях, ограниченных квадриками.

Значение эллиптических биллиардов для геометрии тесно связано с теоремой Понселе, фундаментальным результатом классической проективной геометрии. Как хорошо известно, Жан-Викто́р Понселе доказал ее в 1813 г., после наполеоновских войн с Россией, находясь в плену в Саратове. Его первое доказательство было скорее аналитическим. В 1822 г. Понселе публикует еще одно, чисто геометрическое, синтетическое доказательство в своем Traité des propriétés projectives des figures [2]. Предположим, что на плоскости заданы два эллипса и замкнутая ломаная, вписанная в один из них и описанная около другого. Тогда теорема Понселе утверждает, что существует бесконечно много таких ломаных: любая точка первого эллипса может быть вершиной подобного многоугольника. Вдобавок, у всех таких многоугольников одно и то же число сторон. Затем, в 1828 г., используя теорему сложения для эллиптических функций, К. Якоби дал другое доказательство теоремы Понселе (см. [3]). По существу, теорема Понселе эквивалентна теоремам сложения для эллиптических кривых, и доказательство Понселе дает синтетический метод введения структуры группы на эллиптической кривой. Еще одно доказательство теоремы Понселе на современном алгебро-геометрическом языке было недавно получено Ф. Гриффитсом и Дж. Харрисом (см. [4]). Там же они дали интересное обобщение теоремы Понселе на случай многогранных поверхностей, вписанных и описанных около пары квадрик. При обсуждении истории теоремы Понселе следует отметить замечательное доказательство, данное Г. Дарбу, которое можно найти в [5; гл. 3]. Это доказательство развивает и обобщает идеи Н. Труди [6], [7] и основано на понятии 2-2 соответствия, восходящем к М. Шалю. В работе [8] обсуждается взаимосвязь этого доказательства с теорией многозначных групп.

Ввиду геометрической трактовки структуры группы на кубике (см. рис. 1), возникает вопрос о поиске аналогичной конструкции структуры группы в случае более высокого рода.

Естественный вопрос, возникающий в связи с теоремой Понселе, заключается в нахождении условия, по паре заданных коник определяющего, существует ли $n$-угольник, вписанный в одну из них и описанный около другой. А. Кэли 


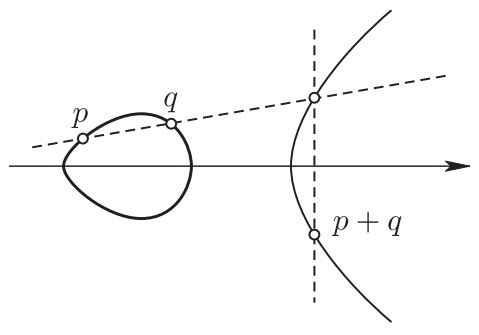

Рис. 1. Групповой закон на кубической кривой

нашел такое условие в 1853 г. в заметке [9], используя теорию абелевых интегралов. Он обсуждал поризм Понселе также в ряде других работ [10]-[14]. Под впечатлением от [9], А. Лебег перевел доказательство Кэли на геометрический язык. Данный Лебегом вывод условия Кэли методами проективной геометрии и алгебры можно найти в его книге Les coniques [15]. В современном контексте Гриффитс и Харрис доказали теорему Кэли, найдя аналитическое условие, задающее точки конечного порядка на эллиптической кривой [16].

Стоит отметит, что на самом деле Понселе доказал намного более общее утверждение, чем общеизвестная теорема Понселе [17], [2], и вывел из него эту теорему как следствие. Точнее говоря, он рассмотрел $n+1$ конику, входящую в пучок на проективной плоскости. Если существует $n$-угольник с вершинами на первой конике, каждая сторона которого касается одной из $n$ оставшихся коник, то таких многоугольников бесконечно много. Мы будем называть это утверждение большой теоремой Понселе, а соответствующие многоугольники многоугольниками Понселе.

Обзор ряда классических результатов типа теоремы Понселе с короткими современными доказательствами дан в [18], а алгебро-геометрический подход с использованием модулярных кривых к семействам многоугольников Понселе представлен в [19], [20].

Перейдем к важной механической интерпретации теоремы Понселе. Эллиптический биллиард [1] - это динамическая система, в которой единичная точечная масса движется по инерции (иными словами, с постоянной скоростью) внутри эллипса, причем на границе выполнен закон отражения: во всякой точке соударения углы падения и отражения с касательной к эллипсу равны. Также предполагается, что отражения абсолютно упруги. Хорошо известно, что всякий отрезок фиксированной биллиардной траектории касается одной и той же коники, софокусной с границей [21]. Если траектория замыкается после $n$ отражений, то по теореме Понселе любая биллиардная траектория с той же каустикой будет периодической, с периодом $n$.

Большая теорема Понселе также имеет механическую интерпретацию. Двойственная конфигурация к пучку коник в плоскости - это семейство софокусных кривых второго порядка [22]. Рассмотрим следующий, немного непривычный биллиард. Пусть даны $n$ софокусных коник, и пусть биллиардный шар последовательно отражается от каждой из этих $n$ коник. Каждый отрезок получившейся траектории касается одной и той же коники, софокусной с данными $n$ кривыми. Если траектория становится замкнутой после $n$ 
отражений, то по большой теореме Понселе всякая подобная траектория с той же каустикой замкнута.

Утверждение, двойственное большой теореме Понселе, можно обобщить на $d$-мерный случай [21]. Предположим, что у многоугольника вершины $x_{1}, \ldots, x_{n}$ расположены на софокусных квадратичных гиперповерхностях $\mathcal{Q}_{1}, \ldots, \mathcal{Q}_{n}$ в $d$-мерном евклидовом пространстве, причем пары соседних сторон удовлетворяют закону отражения от соответствующей гиперповерхности. Тогда все стороны касаются некоторых квадрик $\mathcal{Q}^{1}, \ldots, \mathcal{Q}^{d-1}$, софокусных с $\left\{\mathcal{Q}_{i}\right\}$; для фиксированных наборов $\left\{\mathcal{Q}_{i}, \mathcal{Q}^{j}\right\}$ существует бесконечное семейство многоугольников с этим свойством.

Однако более чем за 100 лет до этих недавних результатов, в 1870 г., Дарбу доказал обобщение теоремы Понселе для биллиарда внутри эллипсоида в трехмерном пространстве [23]. Похоже, его работы в этом направлении сейчас совершенно забыты.

Дарбу занимался теоремой Понселе более 50 лет, и в настоящей работе мы так или иначе развиваем многие из его результатов и идей.

Было бы естественным выяснить условия типа Кэли для обобщений теоремы Понселе. Мы нашли такие условия для биллиарда в эллипсоиде, лежащем в евклидовом пространстве произвольной размерности [24], [25]. Недавно [26]-[29] мы нашли алгебро-геометрические условия существования периодических биллиардных траекторий в системе $k$ квадрик, лежащих в $d$-мерном евклидовом пространстве. Мы излагаем их в п. 2.1 настоящей статьи.

Пункт 2.2 начинается с изложения процедуры алгебро-геометрического интегрирования эллиптического биллиарда; он основан на работе Ю. Мозера и А. Веселова [30]. Затем соответствующие условия типа Кэли выводятся так же, как в [24], [25]. В п. 2.3 приведено изложение по той же схеме для случая геометрии Лобачевского, опирающееся на работы [31] и [32]. Мы завершаем второй раздел статьи пп. 2.4 и 2.5, содержащими результаты из [33] по топологической характеризации изоэнергетических подмногообразий фазового пространства эллиптических биллиардов и по интегрируемым возмущениям потенциалом для таких биллиардов; см. [34]-[38].

Еще одна важная задача нашей статьи - дать систематический обзор истории вопроса, особенно пристально анализируя идеи и результаты Дарбу. В то время как результаты Лебега в этой области, пусть и нечасто, но все же цитируются специалистами, соответствующие идеи Дарбу, как нам представляется, сейчас практически неизвестны математикам. Мы приводим естественные многомерные обобщения идей и результатов Дарбу, равно как и работ Лебега. В числе прочих результатов мы устанавливаем новые интересные свойства пучков квадрик; см. п. 3.1 .

При изложении результатов, развивающих программу Гриффитса и Харриса, мы следуем нашей недавней статье [39]. В п. 3.2 мы вводим обобщенную кривую Кэли, после чего мы приводим геометрическую конструкцию, обобщающую сложение на эллиптической кривой в случае гиперэллиптических якобианов; см. п. 3.3. Наши идеи развивают подходы М. Райда, Р. Донаги и Х. Кнёррера [40]-[42]. Мы развиваем дальше и осуществляем их конструкции, упрощая их и делая наглядными, с помощью идей, развитых в [26] для биллиардных динамических систем на пучках квадрик. 
Основными вехами этого изложения являются работы [41] и [26], а следующее ключевое наблюдение из [39] связывает их: coomветствие $g \mapsto g^{\prime}$ в лемме 4.1 и следствии 4.2 из [41] является биллиардным отображением на квадрике $\mathcal{Q}_{\lambda}$. Подробности см. в следствии 3.15 и сопровождающей его дискуссии.

Таким образом, заметив, что конструкции в [40]-[42] имеют биллиардную основу, и разобравшись в ней, мы получаем возможность использовать аппарат биллиардов для построения и анализа гиперэллиптических якобианов, в особенности их вещественных частей. Всякий вещественный гиперэллиптический якобиан можно реализовать как множество $T$ прямых в $\mathbb{R}^{d}$, касающихся $d-1$ фиксированной квадрики $\mathcal{Q}_{1}, \ldots, \mathcal{Q}_{d-1}$ из некоторого софокусного семейства одновременно. Хорошо известно, что динамическая система, задаваемая квадриками из софокусного семейства, сохраняет такое множество T. Используя теорему о двойном отражении и некоторые другие методы теории биллиардов, мы вводим на $T$ структуру группы, биллиардную алгебру. Использование биллиардов в алгебро-геометрических рассмотрениях, как нередко бывает в таких случаях, оказывается обоюдовыгодным. Мы выводим фундаментальное свойство множества $T$ : любые две прямые из $T$ можно получить друг из друга путем не более чем $d-1$ биллиардного отражения от квадрик из софокусного семейства. Это дает нам возможность ввести в п. 3.4 новые иерархии понятий: $s$-скрещенных прямых из $T, s=-1,0, \ldots, d-2$, и $s$-слабых траекторий Понселе длины $n$. Последние оказываются естественными квазипериодическими обобщениями многоугольников Понселе. Используя биллиардную алгебру, мы полностью описываем эти траектории аналитически. Эти результаты являются дальнейшим обобщением недавно данного нами описания в духе Кэли многоугольников Понселе в произвольной размерности; см. [29]. Мы подчеркнем, что метод, использованный в [39] и основанный на биллиардной алгебре, отличается от методов работы [29]. Ниже мы изложим оба этих метода.

Взаимосвязи между динамикой биллиардов, линейными подпространствами в пересечениях квадрик и гиперэллиптическими якобианами, обсуждаемые в настоящей статье, позволили нам получить многомерные обобщения нескольких классических результатов. Для демонстрации возможностей нашего метода мы приводим в п. 3.5 обобщение теоремы Понселе, принадлежащее Вейру (см. [43]). Мы также выводим и излагаем здесь обобщения на любую размерность пространственной теоремы Понселе, доказанной Гриффитсом и Харрисом (см. [4]). В п. 3.6 мы даем обобщение теоремы Дарбу [44] на произвольную размерность.

Мы с большим удовлетворением посвящаем эту работу академику Валерию Васильевичу Козлову в связи с его 60-летием. Из числа нескольких работ последних 20 лет, в которых Валерий Васильевич обращался к проблематике биллиардов (см., к примеру, [45]-[48]), особый интерес для нашего изложения представляют [38] и [45].

\section{2. Биллиарды в эллипсоидах и их периодические траектории}

Пусть $\mathcal{M}$ - $n$-мерное риманово многообразие и $\Omega \subset \mathcal{M}$ - область с границей, состоящей из нескольких гладких гиперповерхностей. Биллиард в $\Omega$ [1] - это динамическая система, в которой единичная точечная масса свободно движется 
внутри области и подчиняется закону отражения на границе, т. е. углы падения и отражения с касательной к границе во всякой точке соударения равновелики. Предположим также, что отражения происходят абсолютно упруго, так что скорость точечной массы не меняется после соударений.

В п. 2.1 мы рассмотрим случай, когда $\mathcal{M}$ - евклидово пространство, а граница "биллиардного стола" составлена из нескольких софокусных квадрик. Мы изложим наши результаты по аналитическому описанию периодических траекторий в таких биллиардах [26]-[28]. В п. 2.2, с помощью алгебро-геометрического интегрирования дискретной биллиардной системы [30], мы получим соответствующие обобщения условия Кэли [24], [25]. Теорема Понселе и условия Кэли для биллиардов внутри квадрик в пространстве Лобачевского, полученные в [32], излагаются в п. 2.3. После этого, в п. 2.4 мы даем обзор топологических свойств эллиптических биллиардов [33], и завершается раздел результатами по интегрируемым возмущениям таких биллиардов потенциалом [35].

Заметим, что биллиарды в других геометриях также изучались в литературе; к примеру, см. [49]-[51].

2.1. Периодические траектории внутри $k$ софокусных квадрик в евклидовом пространстве. Дарбу был первым, кто рассмотрел многомерное обобщение теоремы Понселе. А именно, он изучал световые лучи в трехмерном случае $(d=3)$ и в 1870 г. анонсировал соответствующую большую теорему Понселе [23].

Обобщения большой теоремы Понселе на более высокие размерности $(d \geqslant 3)$ были получены совсем недавно [21], а соответствующие условия типа Кэли были найдены нами в [26].

Основная задача этого пункта - дать подробное обоснование условия типа Кэли для обобщенной большой теоремы Понселе, а также обсудить его на примерах.

Рассмотрим эллипсоид в $\mathbb{R}^{d}$ :

$$
\frac{x_{1}^{2}}{a_{1}}+\cdots+\frac{x_{d}^{2}}{a_{d}}=1, \quad a_{1}>\cdots>a_{d}>0,
$$

и связанную с ним систему эллиптических координат Якоби $\left(\lambda_{1}, \ldots, \lambda_{d}\right)$, упорядоченных условием

$$
\lambda_{1}>\cdots>\lambda_{d}
$$

Обозначив

$$
Q_{\lambda}(x)=\frac{x_{1}^{2}}{a_{1}-\lambda}+\cdots+\frac{x_{d}^{2}}{a_{d}-\lambda},
$$

мы можем задать всякую квадрику из соответствующего софокусного семейства уравнением вида

$$
\mathcal{Q}_{\lambda} Q_{\lambda}(x)=1
$$

Знаменитая теорема Шаля утверждает, что всякая прямая в $\mathbb{R}^{d}$ касается ровно $d-1$ квадрики из заданного софокусного семейства. В следующей лемме описывается важное свойство этих квадрик.

Лемма 2.1. Пусть прямая $\ell$ касается квадрик $\mathcal{Q}_{\alpha_{1}}, \ldots, \mathcal{Q}_{\alpha_{d-1}}$ из семейства (2.1). Тогда у всякой точки прямой $\ell$ ее координаты Якоби $\left(\lambda_{1}, \ldots, \lambda_{d}\right)$ 
удовлетворяют неравенствам $\mathcal{P}\left(\lambda_{s}\right) \geqslant 0, s=1, \ldots, d$, где

$$
\mathcal{P}(x)=\left(a_{1}-x\right) \cdots\left(a_{d}-x\right)\left(\alpha_{1}-x\right) \cdots\left(\alpha_{d-1}-x\right) .
$$

ДокАЗАТЕЛьство. Пусть $x$ - точка $\ell$ с координатами Якоби $\left(\lambda_{1}, \ldots, \lambda_{d}\right)$, и пусть $y$ - вектор, параллельный $\ell$. Уравнение $Q_{\lambda}(x+t y)=1-$ квадратное по $t$, с дискриминантом

$$
\Phi_{\lambda}(x, y)=Q_{\lambda}(x, y)^{2}-Q_{\lambda}(y)\left(Q_{\lambda}(x)-1\right),
$$

где

$$
Q_{\lambda}(x, y)=\frac{x_{1} y_{1}}{a_{1}-\lambda}+\cdots+\frac{x_{d} y_{d}}{a_{d}-\lambda} .
$$

Согласно [52],

$$
\Phi_{\lambda}(x, y)=\frac{\left(\alpha_{1}-\lambda\right) \cdots\left(\alpha_{d-1}-\lambda\right)}{\left(a_{1}-\lambda\right) \cdots\left(a_{d}-\lambda\right)} .
$$

Для всякой координаты $\lambda=\lambda_{s}(1 \leqslant s \leqslant d)$ это квадратное уравнение имеет решение $t=0$, так что соответствующие дискриминанты неотрицательны. Очевидно, что это эквивалентно условию $\mathcal{P}\left(\lambda_{s}\right) \geqslant 0$.

2.1.1. Биллиард в области, ограниченной софокусными квадриками. Пусть $\Omega \subset \mathbb{R}^{d}$ - ограниченная область с границей $\partial \Omega$, лежащей на объединении нескольких квадрик семейства (2.1). Тогда в эллиптических координатах $\Omega$ можно задать условиями

$$
\beta_{1}^{\prime} \leqslant \lambda_{1} \leqslant \beta_{1}^{\prime \prime}, \quad \ldots, \quad \beta_{d}^{\prime} \leqslant \lambda_{d} \leqslant \beta_{d}^{\prime \prime},
$$

где $a_{s+1} \leqslant \beta_{s}^{\prime}<\beta_{s}^{\prime \prime} \leqslant a_{s}$ при $1 \leqslant s \leqslant d-1$, a $-\infty<\beta_{d}^{\prime}<\beta_{d}^{\prime \prime} \leqslant a_{d}$.

Рассмотрим биллиардную систему в $\Omega$, и пусть $\mathcal{Q}_{\alpha_{1}}, \ldots, \mathcal{Q}_{\alpha_{d-1}}$ - каустики одной из ее траекторий. При всяком $s=1, \ldots, d$ множество $\Lambda_{s}$ значений, принимаемых координатой $\lambda_{s}$ на траектории, согласно лемме 2.1 , содержится в $\Lambda_{s}^{\prime}=\left\{\lambda \in\left[\beta_{s}^{\prime}, \beta_{s}^{\prime \prime}\right]: \mathcal{P}(\lambda) \geqslant 0\right\}$. Согласно [41], во всяком интервале $\left(a_{s+1}, a_{s}\right)$ $(2 \leqslant s \leqslant d)$ лежит не более двух значений из $\alpha_{1}, \ldots, \alpha_{d-1}$, интервал $\left(-\infty, a_{d}\right)$ содержит не более одного из этих чисел, а $\left(a_{1},+\infty\right)$ не содержит ни одного из них. Таким образом, для всякого $s$ возможны три ситуации.

Первыц случай: $\alpha_{i}, \alpha_{j} \in\left[\beta_{s}^{\prime}, \beta_{s}^{\prime \prime}\right], \alpha_{i}<\alpha_{j}$. Поскольку всякая прямая, содержащая отрезок траектории, касается $\mathcal{Q}_{\alpha_{i}}$ и $\mathcal{Q}_{\alpha_{j}}$, вся траектория заключена между двумя этими квадриками. Критические точки эллиптической координатной переменной $\lambda_{s}$ - это точки касания траектории и квадрик, а между ними эллиптическая переменная монотонна. Значит, точки касания с $\mathcal{Q}_{\alpha_{i}}$ и $\mathcal{Q}_{\alpha_{j}}$ чередуются на траектории и $\Lambda_{s}=\Lambda_{s}^{\prime}=\left[\alpha_{i}, \alpha_{j}\right]$.

Bторой случай: из чисел $\alpha_{1}, \ldots, \alpha_{d-1}$ только $\alpha_{i}$ лежит в $\left[\beta_{s}^{\prime}, \beta_{s}^{\prime \prime}\right]$. Многочлен $\mathcal{P}$ неотрицателен ровно на одном из отрезков $\left[a_{s+1}, \alpha_{i}\right]$ и $\left[\alpha_{i}, a_{s}\right]$; будем считать, что на первом. Тогда траектория отражается только от $\mathcal{Q}_{\beta_{s}^{\prime}}$. Если $\alpha_{i} \neq \beta_{s}^{\prime \prime}$, то биллиардный шар никогда на попадает на границу $\mathcal{Q}_{\beta_{s}^{\prime \prime}} . \mathrm{Y}$ переменной $\lambda_{s}$ критические точки - это точки столкновения с $\mathcal{Q}_{\beta_{s}^{\prime}}$ и каустикой $\mathcal{Q}_{\alpha_{i}}$, а вне их она монотонна. Значит, $\Lambda_{s}=\Lambda_{s}^{\prime}=\left[\beta_{s}^{\prime}, \alpha_{i}\right]$. Если $\mathcal{P}$ неотрицателен на $\left[\alpha_{i}, a_{s}\right]$, то $\Lambda_{s}=\Lambda_{s}^{\prime}=\left[\alpha_{i}, \beta_{s}^{\prime \prime}\right]$.

Третий случай: отрезок $\left[\beta_{s}^{\prime}, \beta_{s}^{\prime \prime}\right]$ не содержит значений $\alpha_{1}, \ldots, \alpha_{d-1}$. Тогда $\mathcal{P}$ неотрицателен на $\left[\beta_{s}^{\prime}, \beta_{s}^{\prime \prime}\right]$. Все критические точки $\lambda_{s}$ лежат в точках соприкосновения с граничными квадриками $\mathcal{Q}_{\beta_{s}^{\prime}}$ и $\mathcal{Q}_{\beta_{s}^{\prime \prime}}$, а вне их переменная монотонна. 
Следовательно, биллиардный шар попеременно отскакивает от этих квадрик. Очевидно, что $\Lambda_{s}=\Lambda_{s}^{\prime}=\left[\beta_{s}^{\prime}, \beta_{s}^{\prime \prime}\right]$.

Положим $\left[\gamma_{s}^{\prime}, \gamma_{s}^{\prime \prime}\right]:=\Lambda_{s}=\Lambda_{s}^{\prime}$. Заметим, что для всякой пары $\mathcal{Q}_{\gamma_{s}^{\prime}}, \mathcal{Q}_{\gamma_{s}^{\prime \prime}}$ траектория поочередно наталкивается на эти квадрики. Значит, у всякой периодической траектории число точек пересечения с каждой из этих квадрик одно и то же.

Сделаем несколько замечаний относительно случая, когда $\gamma_{s}^{\prime}=a_{s+1}$ или $\gamma_{s}^{\prime \prime}=a_{s}$. Это означает, что либо часть $\partial \Omega$ является вырожденной квадрикой из софокусного семейства либо $\Omega$ не ограничена хотя бы с одной стороны квадрикой соответствующего вида. Если $\Omega$ ограничена координатной гиперплоскостью, то рассуждения остаются теми же, что и выше. С другой стороны, если часть границы отсутствует, то у координаты $\lambda_{s}$ имеются экстремумы в точках пересечения траектории с соответствующей гиперплоскостью. Поскольку замкнутая траектория должна пересекать гиперплоскость четное число раз, функция $\lambda_{s}$ принимает на периоде каждое из экстремальных значений четное число раз.

ТЕОрема 2.2. Траектория биллиардной системы в $\Omega$ с каустиками $\mathcal{Q}_{\alpha_{1}}$, $\ldots, \mathcal{Q}_{\alpha_{d-1}}$ периодична и имеет ровно $n_{s}$ точек на $\mathcal{Q}_{\gamma_{s}^{\prime}}$ и $n_{s}$ точек на $\mathcal{Q}_{\gamma_{s}^{\prime \prime}}$ $(1 \leqslant s \leqslant d)$ тогда и только тогда, когда

$$
\sum_{s=1}^{d} n_{s}\left(\mathcal{A}\left(P_{\gamma_{s}^{\prime}}\right)-\mathcal{A}\left(P_{\gamma_{s}^{\prime \prime}}\right)\right)=0
$$

на якобиане кривой

$$
\Gamma: y^{2}=\mathcal{P}(x):=\left(a_{1}-x\right) \cdots\left(a_{d}-x\right)\left(\alpha_{1}-x\right) \cdots\left(\alpha_{d-1}-x\right) .
$$

Здесъ $\mathcal{A}$ - отображение Абеля-Якоби, а $P_{\gamma_{s}^{\prime}}, P_{\gamma_{s}^{\prime \prime}}-$ точки на Г с координатами $P_{\gamma_{s}^{\prime}}=\left(\gamma_{s}^{\prime},(-1)^{s} \sqrt{\mathcal{P}\left(\gamma_{s}^{\prime}\right)}\right), P_{\gamma_{s}^{\prime \prime}}=\left(\gamma_{s}^{\prime \prime},(-1)^{s} \sqrt{\mathcal{P}\left(\gamma_{s}^{\prime \prime}\right)}\right)$.

ДокАЗАТЕЛЬСтво. Вслед за Якоби [3] и Дарбу [44] рассмотрим уравнения

$$
\sum_{s=1}^{d} \frac{d \lambda_{s}}{\sqrt{\mathcal{P}\left(\lambda_{s}\right)}}=0, \quad \sum_{s=1}^{d} \frac{\lambda_{s} d \lambda_{s}}{\sqrt{\mathcal{P}\left(\lambda_{s}\right)}}=0, \quad \ldots, \quad \sum_{s=1}^{d} \frac{\lambda_{s}^{d-2} d \lambda_{s}}{\sqrt{\mathcal{P}\left(\lambda_{s}\right)}}=0,
$$

где при фиксированном $s$ квадратный корень $\sqrt{\mathcal{P}\left(\lambda_{s}\right)}$ извлекается с одним и тем же знаком во всех выражениях. Тогда (2.3) представляет собой систему дифференциальных уравнений для прямой, касающейся $\mathcal{Q}_{\alpha_{1}}, \ldots, \mathcal{Q}_{\alpha_{d-1}}$. Кроме того,

$$
\sum_{s=1}^{d} \frac{\lambda_{s}^{d-1} d \lambda_{s}}{\sqrt{\mathcal{P}\left(\lambda_{s}\right)}}=2 d \ell
$$

где $d \ell$ - элемент длины.

Рассматривая всевозможные сочетания знаков $\sqrt{\mathcal{P}\left(\lambda_{1}\right)}, \ldots, \sqrt{\mathcal{P}\left(\lambda_{d}\right)}$, мы получим $2^{d-1}$ неэквивалентных систем $(2.3)$, соответствующих $2^{d-1}$ различным прямым, проходящим через точку общего положения и касающимся $\mathcal{Q}_{\alpha_{1}}, \ldots$, $\mathcal{Q}_{\alpha_{d-1}}$. Более того, системы, соответствующие прямой и ее отражению относительно фиксированной гиперплоскости $\lambda_{s}=$ const, отличаются только знаками радикалов $\sqrt{\mathcal{P}\left(\lambda_{s}\right)}$. 


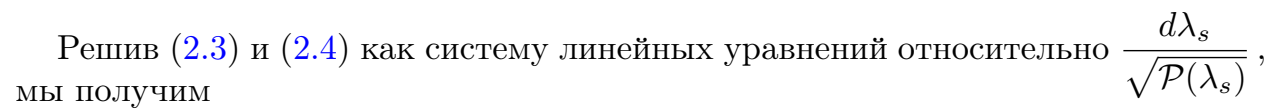

$$
\frac{d \lambda_{s}}{\sqrt{\mathcal{P}\left(\lambda_{s}\right)}}=\frac{2 d \ell}{\prod_{i \neq s}\left(\lambda_{s}-\lambda_{i}\right)}
$$

Таким образом, вдоль биллиардной траектории дифференциалы $\frac{(-1)^{s-1} d \lambda_{s}}{\sqrt{\mathcal{P}\left(\lambda_{s}\right)}}$ остаются положительными, если считать, что знаки квадратных корней выбраны соответствующим образом на каждом интервале.

Из этих замечаний и рассуждений, предшествующих теореме, следует, что интеграл $\int \frac{\lambda_{s}^{i} d \lambda_{s}}{\sqrt{\mathcal{P}\left(\lambda_{s}\right)}}$, взятый по отрезку между двумя соседними точками пересечения траектории и квадрики $\mathcal{Q}_{\gamma_{s}^{\prime}}\left(\right.$ или $\left.\mathcal{Q}_{\gamma_{s}^{\prime \prime}}\right)$, равен

$$
2(-1)^{s-1} \int_{\gamma_{s}^{\prime}}^{\gamma_{s}^{\prime \prime}} \frac{\lambda_{s}^{i} d \lambda_{s}}{+\sqrt{\mathcal{P}\left(\lambda_{s}\right)}}
$$

Теперь если $\mathbf{p}$ - конечный многоугольник, представляющий биллиардную траекторию, у которого ровно $n_{s}$ точек лежат на $\mathcal{Q}_{\gamma_{s}^{\prime}}$ и $n_{s}$ точек лежат на $\mathcal{Q}_{\gamma_{s}^{\prime \prime}}$ $(1 \leqslant s \leqslant d)$, то

$$
\sum \int^{\mathbf{p}} \frac{\lambda_{s}^{i} d \lambda_{s}}{\sqrt{\mathcal{P}\left(\lambda_{s}\right)}}=2 \sum(-1)^{s-1} n_{s} \int_{\gamma_{s}^{\prime}}^{\gamma_{s}^{\prime \prime}} \frac{\lambda_{s}^{i} d \lambda_{s}}{+\sqrt{\mathcal{P}\left(\lambda_{s}\right)}} \quad(1 \leqslant i \leqslant d) .
$$

Далее, ломаная оказывается замкнутой тогда и только тогда, когда

$$
\sum(-1)^{s} n_{s} \int_{\gamma_{s}^{\prime}}^{\gamma_{s}^{\prime \prime}} \frac{\lambda_{s}^{i} d \lambda_{s}}{\sqrt{\mathcal{P}\left(\lambda_{s}\right)}}=0 \quad(1 \leqslant i \leqslant d-1)
$$

что и требовалось доказать.

ПримеР 2.3. Рассмотрим области $\Omega^{\prime}$ и $\Omega^{\prime \prime}$ в $\mathbb{R}^{3}$. Пусть $\Omega^{\prime}$ ограничена эллипсоидом $\mathcal{Q}_{0}$ и двуполостным гиперболоидом $\mathcal{Q}_{\beta}, a_{2}<\beta<a_{1}$, так что $\Omega^{\prime}$ лежит между полостями $\mathcal{Q}_{\beta}$. С другой стороны, допустим, что $\Omega^{\prime \prime}$ ограничена $\mathcal{Q}_{0}$, правой полостью $\mathcal{Q}_{\beta}$ (которая лежит в полупространстве $x_{1}>0$ ) и плоскостью $x_{3}=0$. Эллиптические координаты точек внутри $\Omega^{\prime}$ и $\Omega^{\prime \prime}$ удовлетворяют условиям

$$
0 \leqslant \lambda_{3} \leqslant a_{3}, \quad \beta \leqslant \lambda_{1} \leqslant a_{1} .
$$

В этих областях рассмотрим биллиардные траектории с каустиками $\mathcal{Q}_{\mu_{1}}$ и $\mathcal{Q}_{\mu_{2}}$, $a_{3}<\mu_{1}<a_{2}, a_{2}<\mu_{2}<a_{1}$. Поскольку $\mu_{2} \leqslant \beta$, интервалы $\Lambda_{s}(s \in\{1,2,3\})$ возможных значений эллиптических координат на траектории в обоих случаях следующие:

$$
\Lambda_{1}=\left[\beta, a_{1}\right], \quad \Lambda_{2}=\left[\mu_{1}, a_{2}\right], \quad \Lambda_{3}=\left[0, a_{3}\right] .
$$

В случае $\Omega^{\prime \prime}$ существование периодической траектории с каустиками $\mathcal{Q}_{\mu_{1}}$ и $\mathcal{Q}_{\mu_{2}}$, у которой на периоде $n$ отражений от $\mathcal{Q}_{0}$ и $2 m$ отражений от $\mathcal{Q}_{\beta}$, эквивалентно равенству

$$
n\left(\mathcal{A}\left(P_{0}\right)-\mathcal{A}\left(P_{a_{3}}\right)\right)+2 m\left(\mathcal{A}\left(P_{\beta}\right)-\mathcal{A}\left(P_{\mu_{1}}\right)\right)=0
$$


на якобиане соответствующей эллиптической кривой. В случае $\Omega^{\prime}$ существование траектории с такими свойствами эквивалентно условиям

$$
n\left(\mathcal{A}\left(P_{0}\right)-\mathcal{A}\left(P_{a_{3}}\right)\right)+2 m\left(\mathcal{A}\left(P_{\beta}\right)-\mathcal{A}\left(P_{\mu_{1}}\right)\right)=0 \quad \text { и } \quad n \text { четно. }
$$

То, что из второго равенства следует первое, объясняется следующим геометрическим наблюдением: произвольную биллиардную траекторию в $\Omega^{\prime}$ можно преобразовать в траекторию в $\Omega^{\prime \prime}$, отразив симметрично относительно плоскости $x_{2} x_{3}$ все ее точки, лежащие ниже плоскости. Заметим, что это соответствие траекторий является соответствием "2 к 1", так что биллиардная траектория общего положения в $\Omega^{\prime \prime}$ соответствует ровно двум траекториям в $\Omega^{\prime}$. Примеры таких соответствующих друг другу биллиардных траекторий приведены на рис. 2 , а) и 2 , b).

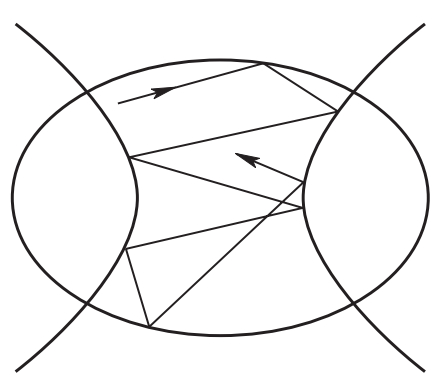

a)

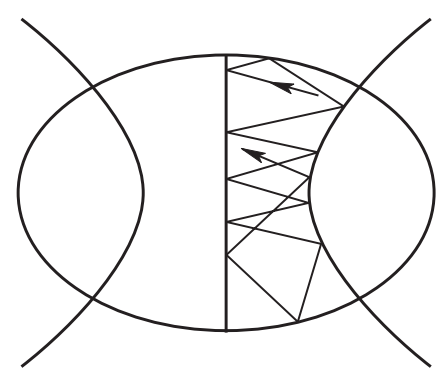

b)

Рис. 2. а) траектория в $\Omega^{\prime}$, b) траектория в $\Omega^{\prime \prime}$

2.1.2. Упорядоченная биллиардная партия. Следующий шаг заключается во введении понятий соударения "снаружи" и "изнутри". Точнее говоря, рассмотрим такой эллипсоид $\mathcal{Q}_{\lambda}$ из софокусного семейства $(2.1)$, что $\lambda \in\left(a_{s+1}, a_{s}\right)$ для некоторого $s \in\{1, \ldots, d\}$, где $a_{d+1}=-\infty$.

Заметим, что после ограничения на биллиардный луч, отражающийся от $\mathcal{Q}_{\lambda}$, у эллиптической переменной $\lambda_{i}$ возникает локальный экстремум в точке отражения.

ОПРЕДЕЛЕНИЕ 2.4. Луч отражается снаружи от квадрики $\mathcal{Q}_{\lambda}$, если точка отражения является локальным максимумом координаты Якоби $\lambda_{s}$, и он отражается изнутри, если точка отражения - локальный минимум $\lambda_{s}$.

На рис. 3 , а) и 3, b) мы схематически изображаем отражения снаружи и изнутри эллипса и гиперболы.

Заметим, что в случае, когда $\mathcal{Q}_{\lambda}$ - эллипсоид, понятия, введенные определением 2.4, имеют обычный смысл.

Пусть дан набор из $k$ софокусных квадрик $\mathcal{Q}_{\beta_{1}}, \ldots, \mathcal{Q}_{\beta_{k}}$ из софокусного пучка $(2.1)$, и пусть $\left(i_{1}, \ldots, i_{k}\right) \in\{-1,1\}^{k}$.

ОПРЕДЕЛЕНИЕ 2.5. Упорядоченная биллиардная партия, ассоциированная с квадриками $\mathcal{Q}_{\beta_{1}}, \ldots, \mathcal{Q}_{\beta_{k}}$, с сигнатурой $\left(i_{1}, \ldots, i_{k}\right)$ - это биллиардная система с траекториями, соударяющимися с $\mathcal{Q}_{\beta_{1}}, \ldots, \mathcal{Q}_{\beta_{k}}$ так, что

при $i_{s}=+1$ отражение от $\mathcal{Q}_{\beta_{s}}$ является отражением изнутри;

при $i_{s}=-1$ отражение от $\mathcal{Q}_{\beta_{s}}$ является отражением снаружи. 


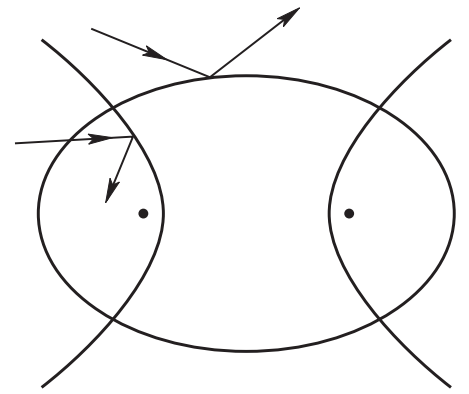

a)

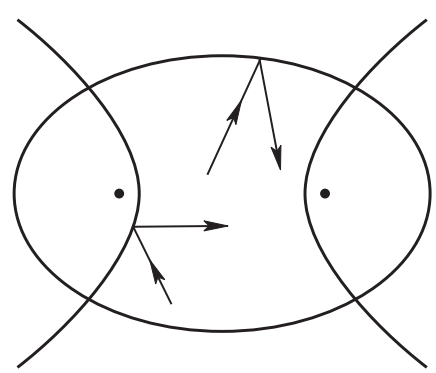

b)

Рис. 3. а) отражение снаружи, b) отражение изнутри

Заметим, что всякая траектория упорядоченной биллиардной партии имеет $d-1$ каустику из того же семейства $(2.1)$.

Пусть $\mathcal{Q}_{\beta_{1}}, \ldots, \mathcal{Q}_{\beta_{k}}$ - эллипсоиды. Рассмотрим упорядоченную биллиардную партию с сигнатурой $\left(i_{1}, \ldots, i_{k}\right)$. Для того чтобы ее траектории были ограничены, должно быть выполнено следующее условие:

$$
i_{s}=-1 \Rightarrow i_{s+1}=i_{s-1}=1 \text { и } \beta_{s+1}<\beta_{s}, \beta_{s-1}<\beta_{s} .
$$

(Здесь отождествляются индексы 0 и $k$, а также $k+1$ и 1.)

ПримеР 2.6. На рис. 4 изображена траектория, соответствующая набору 7 квадрик

$$
\left(\mathcal{Q}_{1}, \mathcal{Q}_{2}, \mathcal{Q}_{1}, \mathcal{Q}_{3}, \mathcal{Q}_{2}, \mathcal{Q}_{3}, \mathcal{Q}_{1}\right)
$$

с сигнатурой $(1,-1,1,-1,1,1,1)$.

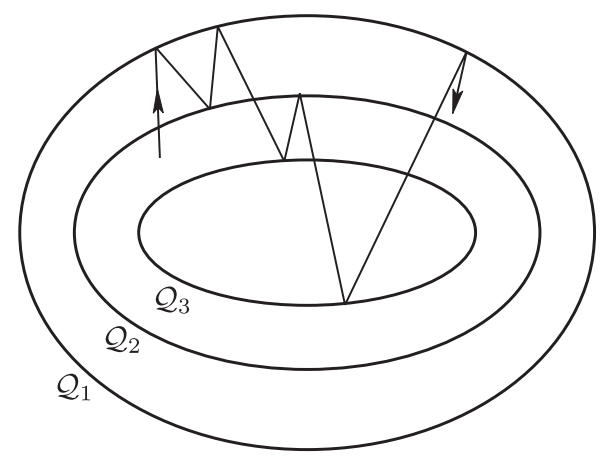

Рис. 4. Упорядоченная биллиардная партия

Теорема 2.7. Пусть дана упорядоченная биллиардная партия с $k$ эллипсоидами $\mathcal{Q}_{\beta_{1}}, \ldots, \mathcal{Q}_{\beta_{k}}$ и с сигнатурой $\left(i_{1}, \ldots, i_{k}\right)$. Ее траектория, имеющая каустики $\mathcal{Q}_{\alpha_{1}}, \ldots, \mathcal{Q}_{\alpha_{d-1}}$, является $k$-периодической, тогда и только тогда, когда сумма

$$
\sum_{s=1}^{k} i_{s}\left(\mathcal{A}\left(P_{\beta_{s}}\right)-\mathcal{A}\left(P_{\alpha}\right)\right)
$$


совпадает с суммой нескольких выражений вида $\mathcal{A}\left(P_{\alpha_{p}}\right)-\mathcal{A}\left(P_{\alpha_{p^{\prime}}}\right)$ на якобиане кривой $\Gamma: y^{2}=\mathcal{P}(x)$, где $P_{\beta_{s}}=\beta_{s},+\sqrt{\mathcal{P}\left(\beta_{s}\right)}, \alpha=\min \left\{a_{d}, \alpha_{1}, \ldots, \alpha_{d-1}\right\}, a \mathcal{Q}_{\alpha_{p}}$, $\mathcal{Q}_{\alpha_{p^{\prime}}}-$ пары каустик одинакового типа

При $\mathcal{Q}_{\beta_{1}}=\cdots=\mathcal{Q}_{\beta_{k}}$ и $i_{1}=\cdots=i_{k}=1$ получаем условие типа Кэли для биллиардной траектории в эллипсоиде из $\mathbb{R}^{d}$.

Разберем подробнее случай биллиардного шара, движущегося между двумя эллипсоидами.

ПРЕДЛОЖЕНИЕ 2.8. Условие существования замкнутой биллиардной траектории, заключенной между эллипсоидами $\mathcal{Q}_{\beta_{1}}$ u $\mathcal{Q}_{\beta_{2}}$, отражающейся ровно $m$ раз от каждого эллипсоида и имеющей каустики $\mathcal{Q}_{\alpha_{1}}, \ldots, \mathcal{Q}_{\alpha_{d-1}}$, имеет следующий вид:

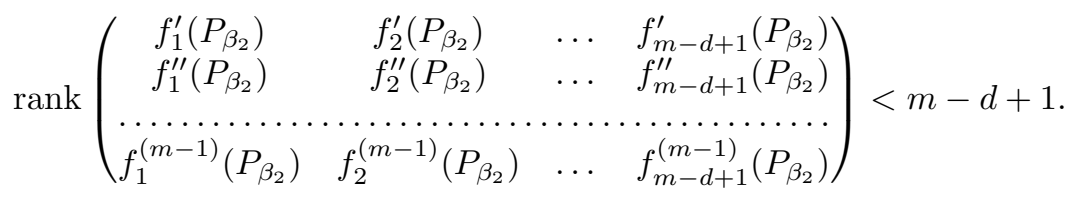

Здесь

$$
f_{j}=\frac{y-B_{0}-B_{1}\left(x-\beta_{1}\right)-\cdots-B_{d+j-2}\left(x-\beta_{1}\right)^{d+j-2}}{x^{d+j-1}}, \quad 1 \leqslant j \leqslant m-d+1,
$$

a $y=B_{0}+B_{1}\left(x-\beta_{1}\right)+\cdots$ разложение в ряд Тейлора в точке, симметричной $P_{\beta_{1}}$ относительно гиперэллиптической инволющии кривой Г. (Обозначения те же, что в теореме 2.7.)

ПримеР 2.9. Рассмотрим биллиардную траекторию в трехмерном пространстве, в области, ограниченной эллипсоидами $\mathcal{Q}_{0}$ и $\mathcal{Q}_{\gamma}\left(0<\gamma<a_{3}\right)$, с каустиками $\mathcal{Q}_{\alpha_{1}}$ и $\mathcal{Q}_{\alpha_{2}}$. Она становится периодической после 4 соударений изнутри с $\mathcal{Q}_{0}$ и 4 соударений снаружи с $\mathcal{Q}_{\gamma}$, тогда и только тогда, когда

$$
\operatorname{rank} X<2 \text {. }
$$

Матрица $X$ имеет вид

$$
\begin{aligned}
& X_{11}=-3 C_{0}+C_{1} \gamma+3 B_{0}+2 B_{1} \gamma+B_{2} \gamma^{2}, \\
& X_{12}=-4 C_{0}+C_{1} \gamma+4 B_{0}+3 B_{1} \gamma+2 B_{2} \gamma^{2}+B_{3} \gamma^{3}, \\
& X_{21}=6 C_{0}-3 C_{1} \gamma+C_{2} \gamma^{2}-6 B_{0}-3 B_{1} \gamma-B_{2} \gamma^{2}, \\
& X_{22}=10 C_{0}-4 C_{1} \gamma-10 B_{0}-6 B_{1} \gamma-3 B_{2} \gamma^{2}-B_{3} \gamma^{3}, \\
& X_{31}=-10 C_{0}+6 C_{1} \gamma-3 C_{2} \gamma^{2}+C_{3} \gamma^{3}+10 B_{0}+4 B_{1} \gamma+B_{2} \gamma^{2}, \\
& X_{32}=-20 C_{0}-10 C_{1} \gamma-4 C_{2} \gamma^{2}+C_{3} \gamma^{3}+20 B_{0}+10 B_{1} \gamma+4 B_{2} \gamma^{2}+B_{3} \gamma^{3},
\end{aligned}
$$

а выражения

$$
\begin{aligned}
& -\sqrt{\left(a_{1}-x\right)\left(a_{2}-x\right)\left(a_{3}-x\right)\left(\alpha_{1}-x\right)\left(\alpha_{2}-x\right)}=B_{0}+B_{1} x+B_{2} x^{2}+\cdots, \\
& +\sqrt{\left(a_{1}-x\right)\left(a_{2}-x\right)\left(a_{3}-x\right)\left(\alpha_{1}-x\right)\left(\alpha_{2}-x\right)}=C_{0}+C_{1}(x-\gamma)+\cdots
\end{aligned}
$$

являются тейлоровскими разложениями в точках $x=0$ и $x=\gamma$ соответственно. 


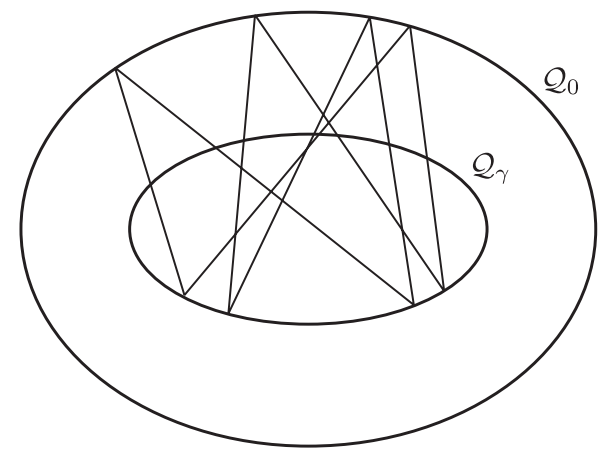

Рис. 5. Замкнутая траектория упорядоченной биллиардной партии, делающая 8 отскоков изнутри попеременно от двух эллипсов

ПримеР 2.10. В тех же обозначениях, что и в предыдущем примере, рассмотрим траектории, четырежды отраженные изнутри от каждого из $\mathcal{Q}_{0}$ и $\mathcal{Q}_{\gamma}$, как на рис. 5 .

Явным условием периодичности подобной траектории является неравенство

$$
\operatorname{rank} X<2
$$

где

$$
\begin{aligned}
& X_{11}=-4 C_{0}+C_{1} \gamma+3 B_{1} \gamma+2 B_{2} \gamma^{2}+B_{3} \gamma^{3}, \\
& X_{12}=-3 C_{0}+C_{1} \gamma+3 B_{0}+2 B_{1} \gamma+B_{2} \gamma^{2}, \\
& X_{21}=-6 C_{0}+C_{2} \gamma^{2}+6 B_{0}+6 B_{1} \gamma+5 B_{2} \gamma^{2}+3 B_{3} \gamma^{3}, \\
& X_{22}=-6 C_{0}+C_{1} \gamma+C_{2} \gamma^{2}+6 B_{0}+5 B_{1} \gamma+3 B_{2} \gamma^{2}, \\
& X_{31}=-4 C_{0}+C_{3} \gamma^{3}+4 B_{0}+4 B_{1} \gamma+4 B_{2} \gamma^{2}+3 B_{3} \gamma^{3}, \\
& X_{32}=-4 C_{0}+C_{2} \gamma^{2}+C_{3} \gamma^{3}+4 B_{0}+4 B_{1} \gamma+3 B_{2} \gamma^{2} .
\end{aligned}
$$

2.2. Биллиарды в эллипсоидах как системы с дискретным временем. Еще один подход к описанию периодических биллиардных траекторий основан на технике дискретных представлений Лакса.

В этом пункте мы сначала перечислим основные этапы алгебро-геометрического интегрирования эллиптических биллиардов согласно [30]. Затем мы установим связь между периодическими биллиардными траекториями и точками конечного порядка на соответствующей гиперэллиптической кривой и найдем условия типа Кэли, следуя в этом нашему изложению в [24], [25]. Кроме того, мы обсудим более подробно траектории с периодом, не превосходящим $d$, и случай сингулярной изоспектральной кривой.

Следуя [30], мы рассмотрим биллиардную систему как систему с дискретным временем. Используя алгоритм интегрирования такой системы, мы установим связь между периодическими биллиардными траекториями и точками конечного порядка на соответствующей гиперэллиптической кривой. 
2.2.1. XYZ-модель и изоспектральные кривые.

Эллиптический биллиард как механическая система с дискретным временем. Рассмотрим эллипсоид в $\mathbb{R}^{d}$, заданный уравнением

$$
(A x, x)=1 .
$$

Будем считать, что $A$ - диагональная матрица с различными собственными значениями. Движение биллиардного шара внутри эллипсоида описывается уравнениями

$$
x_{k+1}-x_{k}=\mu_{k} y_{k+1}, \quad y_{k+1}-y_{k}=\nu_{k} A x_{k},
$$

где

$$
\mu_{k}=-\frac{2\left(A y_{k+1}, x_{k}\right)}{\left(A y_{k+1}, y_{k+1}\right)}, \quad \nu_{k}=-\frac{2\left(A x_{k}, y_{k}\right)}{\left(A x_{k}, A x_{k}\right)} .
$$

Здесь $x_{k}$ - точки отражений биллиардного шара, а $y_{k}=\left(x_{k}-x_{k-1}\right) /\left|x_{k}-x_{k-1}\right|-$ импульсы.

Связь между биллиардом и $X Y Z$-моделью. Как показано в [30], с биллиардной системой с дискретным временем можно связать $X Y Z$-модель Гейзенберга. Опишем эту конструкцию. Дискретная $X Y Z$-система Гейзенберга соответствует системе, описывающей стационарные решения $X Y Z$-цепочки Гейзенберга с классическими спинами.

Рассмотрим отображение $\varphi:(x, y) \mapsto\left(x^{\prime}, y^{\prime}\right)$, заданное равенствами

$$
x_{k}^{\prime}=J y_{k+1}=J\left(y_{k}+\nu_{k} A x_{k}\right), \quad y_{k}^{\prime}=-J^{-1} x_{k}, \quad J=A^{-1 / 2} .
$$

Заметим, что динамика $\varphi$ включает в себя биллиардную динамику

$$
x_{k}^{\prime \prime}=J y_{k+1}^{\prime}=-x_{k+1}, \quad y_{k}^{\prime \prime}=-J^{-1} x_{k}^{\prime}=-y_{k+1},
$$

и рассмотрим последовательность $\left(\bar{x}_{k}, \bar{y}_{k}\right)$, где

$$
\left(\bar{x}_{0}, \bar{y}_{0}\right):=\left(x_{0}, y_{0}\right), \quad\left(\bar{x}_{k+1}, \bar{y}_{k+1}\right):=\varphi\left(\bar{x}_{k}, \bar{y}_{k}\right),
$$

для которой выполнены соотношения

$$
\bar{x}_{k+1}=J \bar{y}_{k}+\nu_{k} J^{-1} \bar{x}_{k}, \quad \bar{y}_{k+1}=-J^{-1} \bar{x}_{k}
$$

с параметром $\nu_{k}$, выбранным так, чтобы $\left|\bar{y}_{k}\right|=1,\left(A \bar{x}_{k}, \bar{x}_{x}\right)=1$. Мы можем переписать это следующим образом:

$$
\bar{x}_{k+1}+\bar{x}_{k-1}=\nu_{k} J^{-1} \bar{x}_{k} .
$$

Тогда для последовательности $q_{k}:=J^{-1} \bar{x}_{k}$ получаем

$$
q_{k+1}+q_{k-1}=\nu_{k} J^{-1} q_{k}, \quad\left|q_{k}\right|=1 .
$$

Эти соотношения представляют собой уравнения дискретной $X Y Z$-системы Гейзенберга.

ТеОрема 2.11 [30]. Пусть $\left(\bar{x}_{k}, \bar{y}_{k}\right)$ - последовательность, связанная с эллиптическим биллиардом вышеописанным образом. Тогда $q_{k}=J^{-1} \bar{x}_{k}-$ решение дискретной системы Гейзенберга. 
Наоборот, если $q_{k}$ - решение системы Гейзенберга, то последовательность $x_{k}=(-1)^{k} J q_{2 k}$ является траекторией дискретного биллиарда внутри эллипсоида.

Интегрирование дискретной $X Y Z$-системы Гейзенберга. Обычный алгоритм алгебро-геометрического интегрирования заключается в следующем [30]. Сначала надо задать последовательность $L_{k}(\lambda)$ матричнозначных полиномов вместе с разложением на множители

$$
L(\lambda)=B(\lambda) C(\lambda) \mapsto C(\lambda) B(\lambda)=B^{\prime}(\lambda) C^{\prime}(\lambda)=L^{\prime}(\lambda)
$$

так, чтобы динамика $L \mapsto L^{\prime}$ соответствовала динамике системы $q_{k}$. Для каждой задачи такая система ищется индивидуально и требует от исследователя хорошей интуиции. Матрицы $L_{k}$ подобны между собой и задают одну и ту же изоспектралъную кривую

$$
\Gamma: \operatorname{det}(L(\lambda)-\mu I)=0 .
$$

Разложение $L_{k}=B_{k} C_{k}$ задает разложение спектра $L_{k}$. Обозначим через $\psi_{k}$ соответствующие собственные векторы и будем рассматривать их как мероморфные функции на Г. Обозначим соответствующие дивизоры полюсов через $D_{k}$.

Последовательность дивизоров линейна на якобиане изоспектральной кривой, и это позволяет восстановить собственные функции $\psi_{k}$, затем матрицы $L_{k}$ и, наконец, последовательность $\left(q_{k}\right)$.

Теперь мы вкратце изложим процедуру интегрирования $X Y Z$-системы этим методом. Детали можно найти в [30].

Уравнения дискретной $X Y Z$-модели эквивалентны изоспектральной деформации:

$$
L_{k+1}(\lambda)=A_{k}(\lambda) L_{k}(\lambda) A_{k}^{-1}(\lambda)
$$

где

$$
\begin{gathered}
L_{k}(\lambda)=J^{2}+\lambda q_{k-1} \wedge J q_{k}-\lambda^{2} q_{k-1} \otimes q_{k-1}, \\
A_{k}(\lambda)=J-\lambda q_{k} \otimes q_{k-1} .
\end{gathered}
$$

Уравнение изоспектральной кривой $\Gamma: \operatorname{det}(L(\lambda)-\mu I)=0$ может быть записано в следующем виде:

$$
\nu^{2}=\prod_{i=1}^{d-1}\left(\mu-\mu_{i}\right) \prod_{j=1}^{d}\left(\mu-J_{j}^{2}\right)
$$

где $\nu=\lambda \prod_{i=1}^{d-1}\left(\mu-\mu_{i}\right)$, а $\mu_{1}, \ldots, \mu_{d-1}-$ нули функции

$$
\begin{gathered}
\phi_{\mu}(x, J y)=\sum_{i=1}^{d} \frac{F_{i}(x, y)}{\mu-J_{i}^{2}}, \\
F_{i}=x_{i}^{2}+\sum_{j \neq i} \frac{(x \wedge J y)_{i j}^{2}}{J_{i}^{2}-J_{j}^{2}}, \quad x=q_{k-1}, \quad y=q_{k} .
\end{gathered}
$$

Можно доказать, что $\mu_{1}, \ldots, \mu_{d-1}$ - параметры каустики, соответствующей биллиардной траектории [52]. К этому же выводу можно прийти, если вычислить эти параметры явно, рассмотрев биллиардную траекторию, начальный отрезок которой параллелен координатной оси. 
Зная собственные векторы $\psi_{k}$ матриц $L_{k}(\lambda)$, можно единственным образом определить элементы последовательности $\left(q_{k}\right)$. Пусть $D_{k}$ - дивизор полюсов функции $\psi_{k}$ на кривой Г. Тогда [30]

$$
D_{k+1}=D_{k}+P_{\infty}-P_{0},
$$

где $P_{\infty}$ - точка, соответствующая значению $\mu=\infty$, а $P_{0}$ соответствует $\mu=0$, $\lambda=\left(q_{k}, J^{-1} q_{k+1}\right)^{-1}$.

2.2.2. Характеризация периодических биллиардных траекторий. В следующих леммах устанавливается связь между периодическими биллиардными последовательностями $q_{k}$ и периодическими дивизорами $D_{k}$.

Лемма 2.12 [24]. Последовательность дивизоров $D_{k}$ периодична с периодом $n$ тогда и только тогда, когда либо последовательность $q_{k}$ также периодична с периодом $n$, либо $q_{k+n}=-q_{k}$ для любого $k$.

ДокАЗАТЕЛЬСтво. Если для всех $k$ имеем $q_{k+n}=q_{k}$ или если $q_{k+n}=-q_{k}$ для всех $k$, то, очевидно, $L_{k+n}=L_{k}$. Так что последовательность собственных векторов $\psi_{k}$ периодична с периодом $n$. Значит, последовательность дивизоров тоже периодична.

Теперь предположим, что $D_{k+n}=D_{k}$ для всех $k$ и, следовательно, $\psi_{k+n}=$ $c_{k} \psi_{k}$. Тогда

$$
\psi_{k+1}=A_{k}(\lambda) \psi_{k}
$$

Пусть $\mu_{1}$ и $\mu_{2}$ - значения параметра $\mu$, соответствующие $\lambda=1$ на кривой $\Gamma$, и пусть

$$
\Psi_{k}=\left(\psi_{k}\left(1, \mu_{1}\right), \psi_{k}\left(1, \mu_{2}\right)\right) .
$$

Из равенства $\psi_{k+1}=A_{k}(\lambda) \psi_{k}$ получаем, что $A_{k}(1)=\Psi_{k+1} \Psi_{k}^{-1}$. Следовательно,

$$
A_{k}(1)=\frac{c_{k+1}}{c_{k}} A_{k+n}(1)
$$

Из условия, что $\operatorname{det} A_{k}=\operatorname{det} A_{k+1}$ для всех $k$, имеем $c_{k}=c_{k+1}$. Значит, последовательность

$$
A_{k}(1)=J-q_{k} \otimes q_{k-1}
$$

$n$-периодическая. Отсюда видно, что

$$
q_{k+n}=\alpha_{k} q_{k}, \quad q_{k+n-1}=\frac{1}{\alpha_{k}} q_{k-1} .
$$

Поскольку $\left|q_{k}\right|=1$, то выполнено $\alpha_{k}=1$ или $\alpha_{k}=-1$, где все $\alpha_{k}$ равны между собой. Это доказывает нужное утверждение.

Лемма 2.13 [24]. Биллиардная траектория периодична с периодом $n$ с точностъю до централъной симметрии тогда и только тогда, когда последовательность дивизоров $D_{k}$, сопоставленная соответствующей $X Y Z$-системе Гейзенберга, периодическая с периодом 2 .

ДокаЗАтельство. Пусть $x_{k+n}=\alpha x_{k}$ для всех $k$ и $\alpha \in\{-1,1\}$. Для биллиардной траектории $\left(x_{k}, y_{k}\right)$ рассмотрим соответствующий поток $\left(\bar{x}_{k}, \bar{y}_{k}\right)$. Поскольку

$$
\left(\bar{x}_{2 k}, \bar{y}_{2 k}\right)=(-1)^{k}\left(x_{k}, y_{k}\right), \quad\left(\bar{x}_{2 k+1}, \bar{y}_{2 k+1}\right)=\phi\left(\bar{x}_{2 k}, \bar{y}_{2 k}\right),
$$


где $\phi$ - линейное отображение, мы видим, что

$$
\bar{x}_{k+2 n}=\alpha(-1)^{n} \bar{x}_{k} .
$$

Отсюда непосредственно следует, что $q_{k+2 n}=\alpha(-1)^{n} q_{k}$. По лемме 2.12 последовательность дивизоров $D_{k}$ является $2 n$-периодической.

Используя эту лемму, докажем основной результат настоящего пункта.

Теорема 2.14 [25]. Если биллиардная траектория в эллипсоиде $\mathcal{Q}_{0}$ из $\mathbb{R}^{d}$ с невырожденными каустиками $\mathcal{Q}_{\mu_{1}}, \ldots, \mathcal{Q}_{\mu_{-1}}$ периодична с точностью до центральной симметрии, с периодом $n \geqslant d$, то выполнено следующее условие:

$$
\operatorname{rank}\left(\begin{array}{cccc}
B_{n+1} & B_{n} & \ldots & B_{d+1} \\
B_{n+2} & B_{n+1} & \ldots & B_{d+2} \\
\ldots \ldots & \ldots \ldots \ldots \ldots & \ldots \ldots \\
B_{2 n-1} & B_{2 n-2} & \ldots & B_{n+d-1}
\end{array}\right)<n-d+1,
$$

¿де

$$
\sqrt{\left(x-\mu_{1}\right) \cdots\left(x-\mu_{d-1}\right)\left(x-a_{1}\right) \cdots\left(x-a_{d}\right)}=B_{0}+B_{1} x+B_{2} x^{2}+\cdots .
$$

ДокАЗАТЕЛЬСтво. По лемме 2.13 траектория будет периодической с периодом $n$, если соответствующая последовательность дивизоров $Г$ имеет период $2 n$, т. е. $2 n\left(P_{\infty}-P_{0}\right)=0$ на якобиане $\mathcal{J}(\Gamma)$. Кривая Г гиперэллиптическая, рода $g=d-1$. Взяв $\mathcal{A}\left(P_{\infty}\right)$ равным нулю на $\mathcal{J}(\Gamma)$, получаем требуемое.

Случай сингулярной изоспектральной кривой. $\mathrm{B}$ случае, когла все $a_{1}, \ldots$, $a_{d}, \mu_{1}, \ldots, \mu_{d-1}$ различны, у изоспектральной кривой нет особенностей в аффинной части. Однако особенности возникают в следующих трех случаях (и при их сочетании).

(i) $a_{i}=\mu_{j}$ для некоторых $i$ и $j$. Изоспектральная кривая (2.5) распадается на рациональную и гиперэллиптическую. Геометрически это означает, что каустика, соответствующая $\mu_{i}$, вырождается в гиперплоскость $x_{i}=0$. Биллиардная траектория будет стремиться к этой плоскости асимптотически (и, значит, не может быть периодической) или лежит целиком в указанной гиперплоскости. Таким образом, замкнутые траектории возникают при условии, что они лежат в координатной гиперплоскости. Такие движения можно рассматривать как случай размерности $d-1$.

(ii) $a_{i}=a_{j}$ при некотором $i \neq j$. Граница $\mathcal{Q}_{0}$ симметрична.

(iii) $\mu_{i}=\mu_{j}$ при некотором $i \neq j$. Биллиардная траектория лежит на соответствующей софокусной квадратичной гиперповерхности.

В случаях (ii) и (iii) изоспектральная кривая Г является гиперэллиптической кривой с особенностями. При всем различии геометрической природы, в обоих этих случаях нужно провести один и тот же анализ условия $2 n P_{0} \sim 2 n P_{\infty}$ для сингулярной кривой (2.5).

Используя нормализацию как в [29], можно показать, что теорема 2.14 применима не только в случае гладкой изоспектральной кривой, но и в случаях (ii) и (iii). Таким образом, имеет место следующее интересное свойство. 
Теорема 2.15. Если биллиардная траектория в эллипсоиде из d-мерного евклидова пространства периодическая с периодом $n<d$ с точностью до центральной симметрии, то она лежит на одной из $n$-мерных плоскостей симметрии эллипсоида.

ДокАЗАТЕЛЬство. Это непосредственно вытекает из теоремы 2.14 и того факта, что при пересечении софокусного семейства квадрик с координатной гиперплоскостью снова получается софокусное семейство.

Заметим, что все траектории, имеющие период $n$ с точностью до центральной симметрии, становятся замкнутыми после $2 n$ отражений. Более точный результат, чем теорема 2.15 , можно элементарно доказать для траекторий, становящихся замкнутыми после $n$ отскоков, где $n \leqslant d$.

ПрЕДЛОЖЕНИЕ 2.16. Если биллиардная траектория в эллипсоиде из $d$-мерного евклидова пространства периодична с периодом $n \leqslant d$, то она лежит на одной из $(n-1)$-мерных плоскостей симметрии эллипсоида.

ДокАЗАтельство. Рассмотрим сначала случай $n=d$. Пусть $x_{1} \ldots x_{d}-$ периодическая траектория, а $(N, x)=\alpha$ - уравнение гиперплоскости, проходящей через ее вершины (здесь $N$ - нормальный вектор к гиперплоскости, а $\alpha-$ константа). Поскольку все нормали к поверхности эллипсоида в точках отражения лежат в этой гиперплоскости, имеем $\left(A N, x_{i}\right)=\left(N, A x_{i}\right)=0$. Итак, $(A N, x)=0$ - тоже уравнение этой гиперплоскости, значит, $\alpha=0$ и векторы $N$ и $A N$ коллинеарны. Это немедленно приводит к требуемому результату.

Случай $n<d$ можно доказать аналогично или используя теорему 2.15 и предыдущий случай $n=d$.

В случае $d=3$ это свойство наглядно видно.

ПримеР 2.17. Рассмотрим движение биллиардного шара в эллипсоиде из трехмерного пространства с $\mu_{1}=\mu_{2}$, когда отрезки траектории лежат на образующих соответствующего однополостного гиперболоида, софокусного с эллипсоидом. Если бы существовала периодическая траектория с периодом $n=$ $d=3$, то три точки отскока лежали бы в одной плоскости и пересечение этой плоскости с квадрикой состояло бы из трех прямых, что невозможно. Очевидно, любая периодическая траектория с периодом $n=2$ проходит по одной из осей эллипсоида. Таким образом, не существует траекторий, лежащих на софокусной квадрике и имеющих период не больше 3.

2.3. Теорема Понселе и условие Кэли в пространстве Лобачевского. А. П. Веселов [31] доказал интегрируемость биллиардной системы в эллипсоиде из пространства Лобачевского. Он показал, что движения в такой системе соответствуют определенным сдвигам якобиана некоторой гиперэллиптической кривой, и привел явные формулы движения в терминах тэта-функций. Самая первая по времени найденная нами ссылка на теорему Понселе в пространстве Лобачевского - это [53].

Цель этого пункта - представить аналог теорем Понселе и Кэли для движений биллиардного шара в эллипсоиде из пространства Лобачевского [32]. 
2.3.1. Интегрирование движения биллиардного шара в пространстве Лобачевского. Для краткого изложения результатов Веселова по биллиардам в пространстве Лобачевского [31] рассмотрим $(d+1)$-мерное пространство Минковского $V=\mathbb{R}^{d, 1}$ с симметричной билинейной формой

$$
\langle\xi, \eta\rangle=-\xi_{0} \eta_{0}+\xi_{1} \eta_{1}+\cdots+\xi_{d} \eta_{d}
$$

Полость гиперболоида $\langle\xi, \xi\rangle=-1$ с индуцированной метрикой является моделью $d$-мерного пространства Лобачевского $\mathbb{H}^{d}$. Эллипсоид Г в таком пространстве задается уравнением

$$
\mathcal{E}=\left\{\xi \in \mathbb{H}^{d}:-\frac{\xi_{0}^{2}}{a_{0}}+\frac{\xi_{1}^{2}}{a_{1}}+\cdots+\frac{\xi_{d}^{2}}{a_{d}}=0\right\}
$$

где $a_{0}>a_{1} \geqslant \cdots \geqslant a_{d}>0$.

Все отрезки биллиардной траектории в этом эллипсоиде касаются (считая кратности) $d-1$ софокусной квадратичной поверхности, соответствующей данной траектории ([31; теорема 3$])$. Пусть $\mu_{i}, i=1, \ldots, d-1,-$ параметры, для которых уравнения этих каустик имеют вид

$$
-\frac{x_{0}^{2}}{a_{0}-\mu_{i}}+\frac{x_{1}^{2}}{a_{1}-\mu_{i}}+\cdots+\frac{x_{d}^{2}}{a_{d}-\mu_{i}}=0 \quad(1 \leqslant i \leqslant d-1) .
$$

Тогда точки отражения от границы $\mathcal{E}$ соответствуют сдвигу $D_{k+1}=D_{k}+$ $Q_{-}-Q_{+}$на якобиане изоспектральной кривой

$$
\Gamma: \quad\left(\mu-a_{0}\right) \cdots\left(\mu-a_{d}\right)=c \lambda^{2}\left(\mu-\mu_{1}\right) \cdots\left(\mu-\mu_{d-1}\right)
$$

где $c$ - константа, а $Q_{+}, Q_{-}$- точки на $\Gamma$, лежащие над $\mu=0$ (см. теорему 2 в [31]; кривая $\Gamma$ - это изоспектральная кривая рассматриваемой там пары $L-A)$.

Заметим, что в [31] рассмотрен только случай гладкой (гиперэллиптической) кривой Г, однако приведенные там рассуждения годятся и для сингулярного случая.

2.3.2. Теорема Понселе и условия типа Кэли. Пусть задана периодическая биллиардная траектория в эллипсоиде $\mathcal{E}$ из пространства Лобачевского. У всех траекторий с теми же каустиками одна и та же изоспектральная кривая. Если период данной траектории равен $n$, то $n\left(Q_{+}-Q_{-}\right)=0$ на $\mathcal{J}(\Gamma)$, и наоборот. Итак, все такие траектории замыкаются после $n$ отскоков. Таким образом, из результатов Веселова мы можем получить следующую теорему типа Понселе для биллиарда в пространстве Лобачевского.

ПРЕДЛОЖЕНИЕ 2.18 [32]. Пусть дана периодическая биллиардная траектория в эллипсоиде из пространств Лобачевского. Тогда всякая биллиардная траектория с теми же каустиками тоже являтся периодической и имеет тот же период.

Аналитическое условие для периодичности биллиардной траектории, будь у нее особая или неособая изоспектральная кривая, формулируется следующим образом. 
Теорема 2.19 [32]. Условие того, что биллиардная траектория в эллипсоиде (2.6) в d-мерном пространстве Лобачевского, имеющая невырожденные каустики (2.7), периодична с точностъю до иентральной симметрии с периодом $n \geqslant d$, имеет вид

$$
\operatorname{rank}\left[\begin{array}{cccc}
B_{n+1} & B_{n} & \ldots & B_{d+1} \\
B_{n+2} & B_{n+1} & \ldots & B_{d+2} \\
\ldots \ldots \ldots \ldots \ldots \ldots \ldots \ldots \ldots \ldots \ldots \\
B_{2 n-1} & B_{2 n-2} & \ldots & B_{n+d-1}
\end{array}\right]<n-d+1
$$

где $\sqrt{\left(x-a_{0}\right) \cdots\left(x-a_{d}\right)\left(x-\mu_{1}\right) \cdots\left(x-\mu_{d-1}\right)}=B_{0}+B_{1} x+B_{2} x^{2}+\cdots$. Такая траектория не может иметь период менъше $d$.

Случай особой изоспектральной кривой может быть разобран так же, как в евклидовом пространстве (см. предыдущий пункт). Таким образом, в пространстве Лобачевского выполнен полный аналог теоремы 2.15:

ТеОРема 2.20 [32]. Если биллиардная траектория в эллипсоиде из $d$-мерного пространства Лобачевского периодична с периодом $n<d$ с точностью до центральной симметрии, то она лежит на одной из n-мерньх плоскостей симметрии эллипсоида.

В заключение пункта интересно отметить, что условия типа Кэли, полученные в случае пространства Лобачевского, имеют в точности тот же вид, что и для биллиарда в евклидовом пространстве. Это удивительное совпадение объясняется траекторной эквивалентностью эллиптических биллиардов в этих геометриях [32].

Следует отметить, что аналоги теоремы Понселе существуют и в других геометриях. На поверхностях Лиувилля такой аналог сформулирован и доказан Дарбу [44] (см. также [5] для интересного случая, относящегося к сферической геометрии). Теорема Понселе для эллиптических биллиардов на квадриках доказана в [54], а в финслеровой плоскости - в [49], [50].

2.4. Топологические свойства эллиптического биллиарда. Предметом этого пункта является топологическое описание эллиптических биллиардов с использованием графов Фоменко [33]. Подробное изложение этого способа топологической классификации интегрируемых систем можно найти в [55]-[57] и в ссылках, приведенных в этих работах, в то время как здесь мы дадим краткое изложение для неосведомленного читателя. Хотя эллиптические биллиарды на плоскости хорошо изучены, мы увидим, что такое описание позволит по-новому взглянуть на их свойства; в частности, отметим появление неориентируемой периодической траектории, идущей вдоль оси симметрии границы биллиардного стола, в предложении 2.23 (см. рис. 9).

В книге [55] можно найти большое количество графов Фоменко для таких известных интегрируемых систем, как интегрируемые случаи движений твердого тела и интегрируемые геодезические потоки на поверхностях. В настоящей статье мы приводим дополнительные примеры такого рода. Еще некоторые примеры, связанные с почти интегрируемой динамикой, можно найти в [58], а бифуркации слоений Лиувилля для некоторого класса интегрируемых систем с двумя степенями свободы были классифицированы с использованием графов Фоменко в [59]. 
Заметим, что топологические свойства эллиптических биллиардов, рассматриваемых как дискретные динамические системы, недавно были изучены в [60]. Однако здесь мы трактуем биллиарды как системы с непрерывным временем, что позволяет нам использовать аппарат, разработанный Фоменко и его школой.

2.4.1. Изоэнергетические поверхности биллиардных систем. Напомним, что мы считаем, что $\mathcal{M}^{n}$ есть $n$-мерное риманово многообразие, а $\Omega \subset \mathcal{M}$ - область с границей, составленной из нескольких гладких гиперповерхностей. Как уже было сказано вслед за [1], биллиард в $\Omega$ - это динамическая система, в которой единичная точечная масса свободно движется внутри области и следует закону отражения на ее границе: углы падения и отражения с касательным пространством к границе во всякой точке удара равны по величине. Также предполагается, что отражения совершенно упругие.

Важно отметить, что при изменении общей энергии системы изменится только величина вектора скорости точки, в то время как траектории на всех энергетических уровнях одни и те же. Вот почему для полного анализа биллиардной системы можно зафиксировать скорость и исследовать один-единственный уровень энергии.

Изоэнергетическое пространство биллиардной системы в $\Omega$ имеет вид

$$
\begin{gathered}
\mathcal{B}=\left\{(x, v): x \in \Omega, v \in T_{x} \mathcal{M},|v|=1\right\} / \sim, \\
(x, u) \sim(x, v) \Longleftrightarrow x \in \partial \Omega \text { и } u-v \perp T_{x} \partial \Omega .
\end{gathered}
$$

Хотя движение биллиардного шара перестает быть гладким на границе, заметим, что, как ни удивительно, если граница биллиардного стола гладкая, то изоэнергетическая поверхность гладкая. Более того, это многообразие будет гладким и тогда, когда граница состоит из нескольких гладких частей, при условии, что закон отражения можно непрерывно определить в угловых точках. Так и будет в наших примерах, где граница будет лежать на нескольких софокусных кониках, поскольку такие коники взаимно ортогональны. Итак, в точках пересечения поверхностей отражение задается следующим образом: после отражения от негладкой точки границы вектор скорости $v$ меняется на $-v$.

2.4.2. Плоские эллиптические биллиарды. Важным примером интегрируемой биллиардной системы является биллиард внутри эллипса в $\mathbb{E}^{2}$. Эта система интегрируема в силу замечательного элементарного наблюдения: все отрезки траектории касаются одного и того же конического сечения, софокусного с границей [22], [17]. Более того, у всякого интегрируемого биллиарда в плоской области граница должна быть составлена из кусков нескольких софокусных конических сечений [61].

Здесь мы опишем топологию плоских биллиардов с эллиптической границей. Для представления изоэнергетических поверхностей мы используем графы Фоменко [56].

Пусть эллипс на плоскости задан уравнением

$$
\mathcal{E}: \frac{x^{2}}{a}+\frac{y^{2}}{b}=1, \quad a>b>0 .
$$


Семейство конических сечений, софокусных с $\mathcal{E}$, имеет вид

$$
\mathcal{C}_{\mu}: \frac{x^{2}}{a-\mu}+\frac{y^{2}}{b-\mu}=1, \quad \mu \in \mathbb{R} .
$$

Мы рассмотрим биллиардную систему в ограниченной области $\Omega$ в $\mathbb{E}^{2}$ с границей, состоящей из дуг нескольких софокусных конических сечений. Как и во введении, обозначим изоэнергетическую поверхность системы через $\mathcal{B}$.

ПРЕДЛОЖЕНИЕ 2.21. Изоэнергетическая поверхность, соответствующая биллиардной системе внутри эллипса в $\mathbb{E}^{2}$, представляется графом Фоменко на рис. 6.

Функиии вращения, соответствующие ребрам графа, монотонны. Их предель на нижних ребрах равны $\infty$ при стремлении $к \mathbf{A}$-атому и 2 при стремлении к $\mathbf{B}$-атому; на верхнем ребре предел равен $\infty$ при стремлении $к \mathbf{A}$-атому и 1 при стремлении к $\mathbf{B}$-атому.

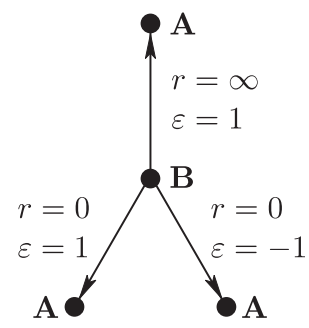

Рис. 6. Граф Фоменко, соответствующий биллиарду внутри эллипса

ДокАЗАТЕЛЬство. Изоэнергетическая поверхность $\mathcal{B}$ является полноторием с отношением эквивалентности $\sim$ на границе, при котором точки попарно идентифицируются. При этом точки двух кривых, представляющих потоки в положительном и отрицательном направлении вдоль границы биллиардного стола, не склеиваются с другими точками. Полноторие с этими двумя кривыми на границе изображено на рис. 7. Две эти кривые соответствуют предельному потоку с каустикой $\mathcal{C}_{0}=\mathcal{E}$; они представляются А-атомами в нижней части графа. При значениях параметра $0<\mu<b$, т. е. когда каустика - эллипс, мы получаем два лиувиллевых тора для всякого значения $\mu$ - по одному на каждое направление вращения.

Опишем теперь множество уровня $\mu=b$. Оно содержит в точности те биллиардные траектории, которые проходят через фокусы эллипса. Одна из этих траекторий периодическая - это движение вдоль оси $x$, а у остальных есть хорошо известное свойство: их отрезки попеременно проходят через левый и правый фокусы эллипса. Эти траектории естественным образом разбиваются на два класса: один класс состоит из траекторий, по которым биллиардный шар движется вверх через левый фокус и вниз через правый, а для траекторий из второго класса движение происходит наоборот. Обозначим эти классы через $\mathcal{S}_{1}$ и $\mathcal{S}_{2}$ соответственно. Траектории из этих классов гомоклинически приближаются к оси $x$, так что $\mathcal{S}_{1}$ и $\mathcal{S}_{2}$ - сепаратрисы. Заметим, что периодическая траектория ориентируема, так что рассматриваемое множество уровня соответствует $\mathbf{B}_{\bar{\Lambda}}$ атому. 


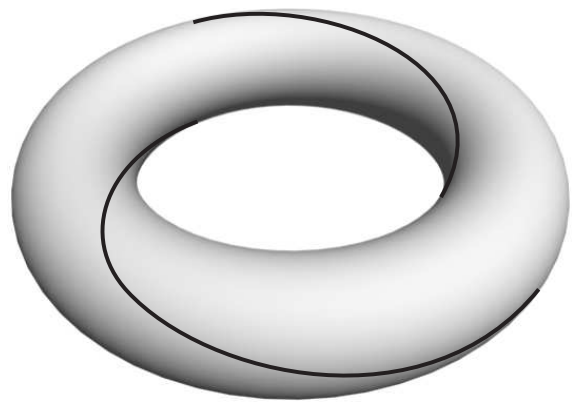

Рис. 7. Изоэнергетическая поверхность для биллиардной системы в эллипсе

Заметим, что $\mathcal{S}_{1}$ (соответственно $\left.\mathcal{S}_{2}\right)$ - предельное множество семейства лиувиллевых торов, соответствующих движению с эллиптической каустикой в направлении по часовой стрелке (соответственно против часовой стрелки).

При $b<\mu<a$, т. е. когда каустика - гипербола, каждому значению параметра соответствует единственный тор. Наконец, при $\mu=a$ происходит периодическое движение вдоль оси $y$; оно изображается $\mathbf{A}_{\bar{\Lambda}}$ атомом сверху.

Аналогичным образом доказывается следующее предложение.

ПРЕДЛОЖЕНИЕ 2.22. Изоэнергетическая поверхность, соответствующая биллиардной системе в области, ограниченной двумя софокусными эллипсами в $\mathbb{E}^{2}$, представляется графом Фоменко, изображенным на рис. 8.
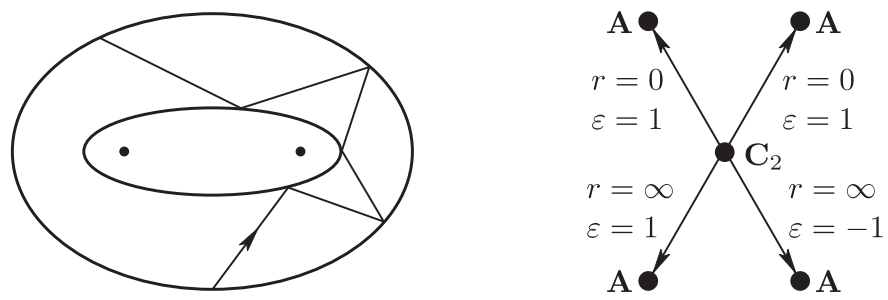

Рис. 8. Биллиард в области, ограниченной софокусными эллипсами, и соответствующий граф Фоменко

Доказательство. Как и в предложении 2.21, $\mathbf{A}_{\bar{\Lambda}}$ атомы в нижней части соответствуют предельным потокам на границе внешнего эллипса.

Множество уровня, отвечающее $\mu=b$, содержит две периодические траектории, лежащие на оси $x$, и все траектории, для которых прямые, содержащие отрезки траекторий, проходят через фокусы. Заметим, что такая траектория гетероклиническая и целиком содержится в одной из полуплоскостей, с границей на оси $x$. Таким образом, в этом множестве уровня четыре сепаратрисы: по две в верхней и нижней полуплоскостях. В каждой полуплоскости одна сепаратриса содержит траектории, приближающиеся к левой периодической траектории на оси абсцисс, когда время возрастает до $\infty$, и к аналогичной правой траектории, когда время убывает до $-\infty$; для траекторий из другой сепаратрисы все происходит наоборот. Лиувиллевы торы, соответствующие 
эллиптическим каустикам, при $\mu \rightarrow b$ сходятся к двум сепаратрисам с тем же направлением движения - по или против часовой стрелки.

В случае гиперболических каустик биллиардный стол разбивается на две области, симметричные относительно оси абсцисс. Каждое из семейств лиувиллевых торов сходится к двум сепаратрисам уровня $\mu=b$, лежащим в той же полуплоскости.

Этот анализ показывает, что множество уровня $\mu=b$ описывается атомом $\mathbb{C}_{2}$.

Атомы А в верхней части графа соответствуют периодическим орбитам, проходящим по оси ординат.

Рассмотрим теперь область, ограниченную эллипсом и софокусной гиперболой. Заметим, что когда граница области является гладкой не во всех точках, фазовое пространство следует описывать особенно тщательно. Поскольку софокусные конические сечения ортогональны друг другу в точках пересечения, периодические потоки вдоль гладких дуг могут быть пределами биллиардных движений при стремлении каустики к коническому сечению, содержащему эту дугу. Хотя касательное пространство к границе в точках пересечения не определено, мы будем считать, что "разрешенные" векторы скорости в этих точках касаются кривых, содержащих эти точки, идентифицируя противоположные векторы.

ПРеДЛОЖЕНИЕ 2.23. Рассмотрим биллиардную область, граница которой образована эллипсом и софокусной гиперболой. Тогда:

1) если эта область лежит внутри одной из “полостей” гиперболь, то изоэнергетическая поверхность представлена графом на рис. 9.

2) Если область лежит между ветвями гиперболь, то изоэнергетическая поверхность представляется графом на рис. 10.
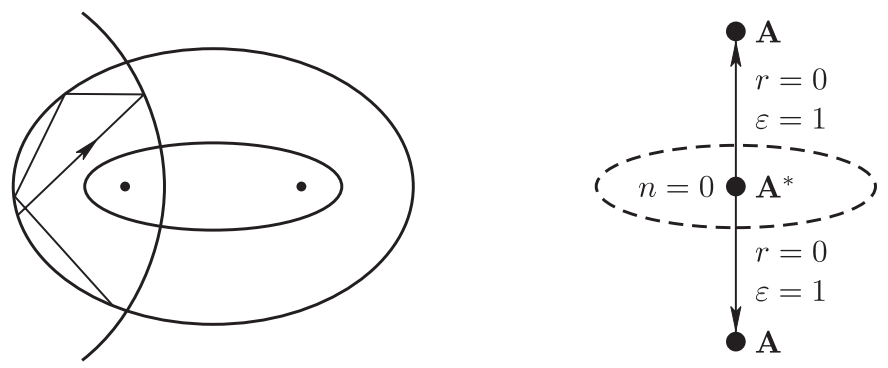

Рис. 9. Биллиард в области между эллипсом и одной из ветвей гиперболы и соответствующий граф Фоменко

ДокАЗАТЕЛЬство. В первом случае каждый из атомов $\mathbf{A}$ соответствует предельной периодической орбите, проходящей по одной из гладких дуг, составляющих границу биллиардной области. Атом $\mathbf{A}^{*}$ представляет периодическую орбиту, проходящую вдоль оси $x$, и ее гомоклинические траектории.

Во втором случае атомы $\mathbf{A}$ внизу графа соответствуют предельному движению вдоль (двух) дуг эллипса, атом $\mathbf{B}$ соответствует периодической орбите, 

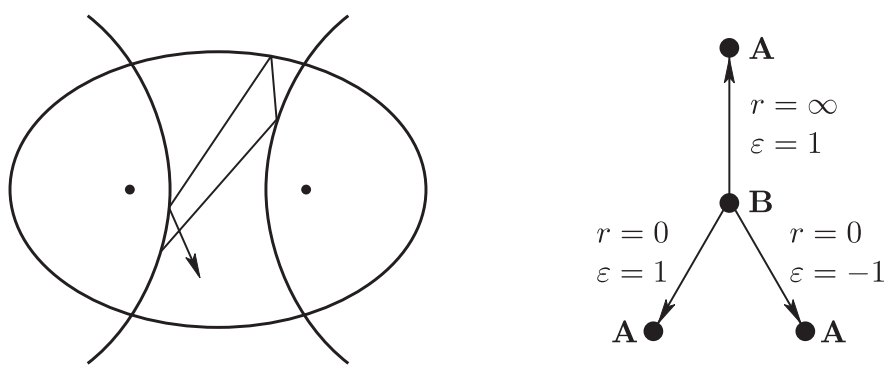

Рис. 10. Биллиард в области между эллипсом и ветвями гиперболы и соответствующий граф Фоменко

проходящей вдоль оси $x$, и ее гомоклиническим траекториям, а атом $\mathbf{A}$ в верхней части соответствует периодической орбите, проходящей по оси $y$. Любопытно отметить, что эта система эквивалентна по Лиувиллю биллиарду внутри эллипса (ср. рис. 6 и 10).

Чтобы понять, как возникают бифуркации изоэнергетических поверхностей, проанализируем пример, в котором граница биллиардного стола непрерывно меняется.

ПРеДЛОЖеНИЕ 2.24. Рассмотрим биллиардную область, ограниченную двумя софокусными эллипсами и дугами разных ветвей софокусной гиперболь.

1) Если область лежит между ветвями гиперболь, то изоэнергетическая поверхность представляется графом на рис. 11.

2) Если осъ абсцисс является частью границы, то изоэнергетическая поверхность представляется графом на рис. 12. (Отметим, что V соответствует вырожденному особому листу, содержащему две ориентируемые периодические траектории и две гетероклинические сепаратрисъь.)

3) Если область соответствует изображению на рис. 13 слева, то изоэнергетическая поверхность представляется графом на рис. 13 справа.
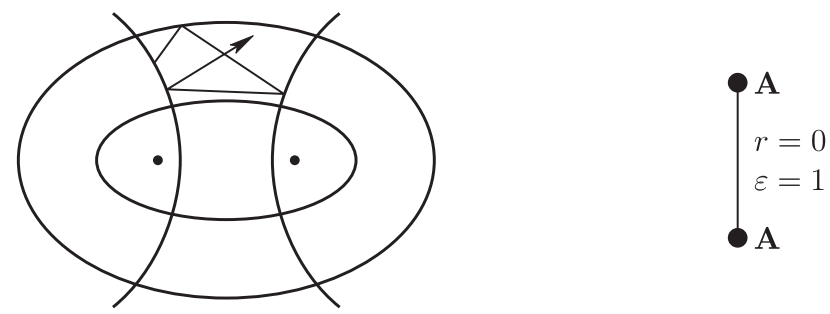

Рис. 11. Биллиардное движение между двумя эллипсами и гиперболой и соответствующий граф Фоменко

ЗАМЕчАНИЕ 2.25. Почти интегрируемые бифуркации биллиардов из предложений 2.23 и 2.24 можно анализировать, используя методы работы [62]. 

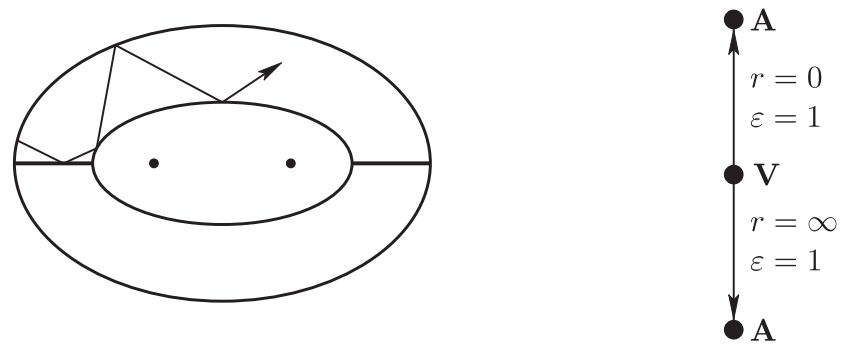

Рис. 12. Биллиардное движение между двумя эллипсами и вырожденной гиперболой и соответствующий граф Фоменко
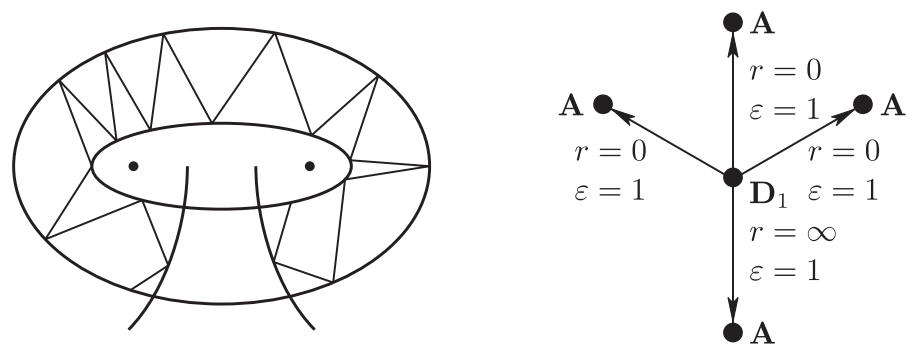

Рис. 13. Биллиардное движение в области между двумя эллипсами гиперболой и соответствующий граф Фоменко

2.4.3. Биллиарды на эллипсоидах и поверхности Лиувилля. В этом пункте мы проанализируем топологию биллиардного движения на эллипсоиде из $\mathbb{E}^{3}$, с границей, высеченной софокусной квадрикой. Затем будут рассмотрены биллиарды внутри обобщенных эллипсов на поверхностях Лиувилля. Мы сравним результаты с полученными в п. 2.4.2.

Топология движений по геодезическим на эллипсоиде из $\mathbb{E}^{3}$. Поскольку отрезки биллиардных траекторий на эллипсоиде лежат на геодезических, имеет смысл рассмотреть топологию изоэнергетических поверхностей для геодезического движения на эллипсоиде. Она полностью описана в [63].

Пусть эллипсоид в $\mathbb{E}^{3}$ задан уравнением

$$
\mathcal{E}: \frac{x^{2}}{a}+\frac{y^{2}}{b}+\frac{z^{2}}{c}=1, \quad 0<c<b<a .
$$

Теорема 2.26 [63], [55]. Граф Фоменко для задачи Якоби о геодезических на эллипсоиде представлен на рис. 14.

Функиии вращения, соответствующие нижним и верхним ребрам графа, имеют вид

$$
\begin{array}{ll}
\rho_{\text {lower }}(\alpha)=\left(\int_{c}^{\alpha} \Phi(\lambda, \alpha) d \lambda\right)^{-1} \int_{b}^{a} \Phi(\lambda, \alpha) d \lambda & (\alpha \in(c, b)), \\
\rho_{\text {upper }}(\alpha)=\left(\int_{c}^{b} \Phi(\lambda, \alpha) d \lambda\right)^{-1} \int_{\alpha}^{a} \Phi(\lambda, \alpha) d \lambda & (\alpha \in(b, a)),
\end{array}
$$


соответственно, где

$$
\Phi(\lambda, \alpha)=\frac{\lambda}{\sqrt{-\lambda(a-\lambda)(b-\lambda)(c-\lambda)(\alpha-\lambda)}} .
$$

Интересно отметить, что этот результат используется в доказательстве того факта, что задача Якоби о геодезических на эллипсоиде и случай Эйлера движения твердого тела траекторно эквивалентны (см. [63], [55]).

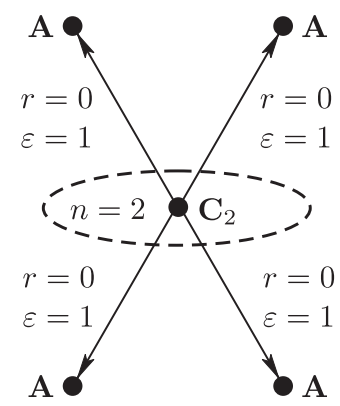

Рис. 14. Граф Фоменко задачи Якоби

Биллиард на эллипсоиде в $\mathbb{E}^{3}$. Обобщенный эллипс на эллипсоиде $(2.9)$ это пересечение эллипсоида с софокусным гиперболоидом. Заметим, что подобное пересечение состоит из двух непересекающихся замкнутых кривых, являющихся линиями кривизны эллипсоида. Эти кривые пересечения разбивают поверхность эллипсоида на три области: две конгруэнтные односвязные и третью, лежащую между ними и не являющуюся односвязной.

Точнее говоря, всякая поверхность, софокусная с эллипсоидом (2.9), задается уравнением вида (см. рис. 15):

$$
\mathcal{Q}_{\lambda}: Q_{\lambda}=\frac{x^{2}}{a-\lambda}+\frac{y^{2}}{b-\lambda}+\frac{z^{2}}{c-\lambda}=1 .
$$

Предположим, что координаты Якоби $\left(\lambda_{1}, \lambda_{2}, \lambda_{3}\right)$, связанные с этой системой софокусных поверхностей второго порядка, упорядочены условием $\lambda_{1}>$ $\lambda_{2}>\lambda_{3}$.

Тогда однополостный гиперболоид $\mathcal{Q}_{\beta}(c<\beta<b)$ вырезает на поверхности $\mathcal{E}$ следующие три области: $\Omega_{1}^{\beta}=\left\{\lambda_{2}>\beta, z>0\right\}, \Omega_{2}^{\beta}=\left\{\lambda_{2}>\beta, z<0\right\}$ и $\Omega_{3}^{\beta}=$ $\left\{\lambda_{2}<\beta\right\}$. Первые две из них симметричны относительно плоскости $x y$, а третья является кольцом, расположенным между ними на $\mathcal{E}$, как показано на рис. 16 .

Аналогичным образом, двуполостный гиперболоид $\mathcal{Q}_{\gamma}(b<\gamma<a)$ задает области $\Omega_{1}^{\gamma}=\left\{\lambda_{1}<\gamma, x>0\right\}, \Omega_{2}^{\gamma}=\left\{\lambda_{1}<\gamma, x<0\right\}$ и $\Omega_{3}^{\gamma}=\left\{\lambda_{1}>\gamma\right\}$, см. рис. 17 .

ПРЕДЛОЖЕНИЕ 2.27. Изоэнергетические поверхности, соответствующие биллиардным системам в областях $\Omega_{1}^{\beta}$ и $\Omega_{1}^{\gamma}$ на $\mathcal{E}$, представляются графом Фоменко на рис. 6. 


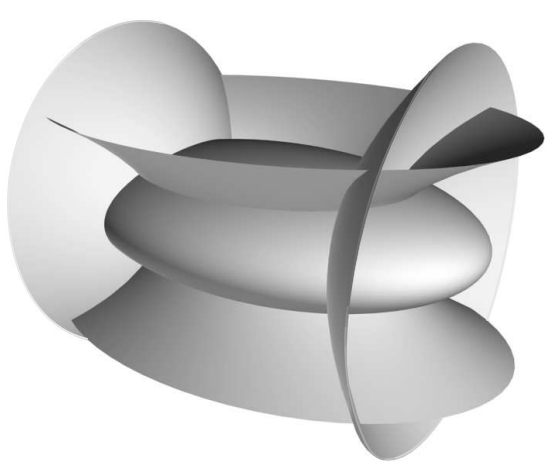

Рис. 15. Софокусные квадрики

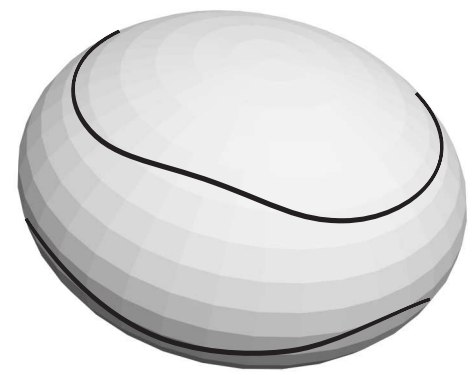

Рис. 16. Пересечение эллипсоида и однополостного гиперболоида

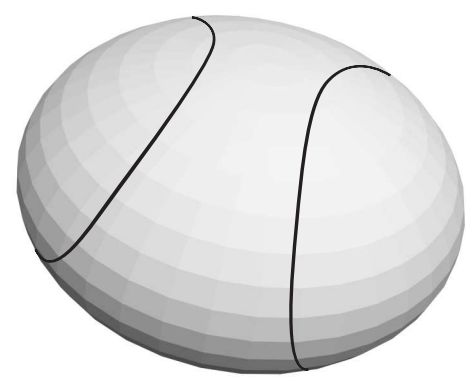

Рис. 17. Пересечение эллипсоида и двуполостного гиперболоида

Функции вращения для нижних и верхних ребер графа в случае биллиарда в $\Omega_{1}^{\beta}$ cyms

$$
\begin{array}{ll}
\rho_{\text {lower }}^{\beta}(\alpha)=\left(\int_{\beta}^{\alpha} \Phi(\lambda, \alpha)\right)^{-1} \int_{b}^{a} \Phi(\lambda, \alpha) & (\alpha \in(\beta, b)), \\
\rho_{\text {upper }}^{\beta}(\alpha)=\left(\int_{\beta}^{b} \Phi(\lambda, \alpha)\right)^{-1} \int_{\alpha}^{a} \Phi(\lambda, \alpha) & (\alpha \in(b, a)),
\end{array}
$$

А функиии вращения для нижних и верхних ребер графа в случае биллиарда в $\Omega_{1}^{\gamma}$ cymb

$$
\begin{array}{ll}
\rho_{\text {lower }}^{\gamma}(\alpha)=\left(\int_{\alpha}^{\gamma} \Phi(\lambda, \alpha)\right)^{-1} \int_{c}^{b} \Phi(\lambda, \alpha) & (\alpha \in(b, \gamma)), \\
\rho_{\text {upper }}^{\gamma}(\alpha)=\left(\int_{b}^{\gamma} \Phi(\lambda, \alpha)\right) \int_{c}^{\alpha} \Phi(\lambda, \alpha) & (\alpha \in(c, b))
\end{array}
$$

(Ф определена в теореме 2.26). 
2.4.4. Биллиард внутри эллипса на поверхности Лиувилля.

ТеОРема 2.28. Все биллиардные системы внутри эллипса на произвольной поверхности Лиувилля эквивалентны по Лиувиллю. Граф Фоменко, соответствующий изоэнергетическим поверхностям такой системы, представлен на рuс. 6.

ДокАзАтельство. Семейства софокусных эллипсов и гипербол, а также соответствующие биллиардные системы в этом случае были определены и описаны Дарбу [44] (см. недавнее изложение этих вопросов в [29], [39]). Поскольку все топологические свойства таких биллиардов аналогичны свойствам биллиардов внутри эллипса в евклидовой плоскости, мы приходим к утверждению теоремы.

2.4.5. Биллиарды внутри эллипсоидов в $\mathbb{E}^{3}$. Теперь мы проанализируем топологию биллиардных движений внутри эллипсоида в $\mathbb{E}^{3}$. Будем считать, что уравнение эллипсоида имеет вид (2.9).

$\mathrm{У}$ всякой траектории биллиардного шара в $\mathcal{E}$ ровно две каустики, принадлежащие софокусному семейству $\mathcal{Q}_{\lambda}$. Пусть $\mathcal{Q}_{\lambda_{1}}$ и $\mathcal{Q}_{\lambda_{2}}, \lambda_{2} \leqslant \lambda_{1}$, - эти поверхности. Тогда бифуркационное множество изоэнергетической поверхности системы изображено на рис. 18.

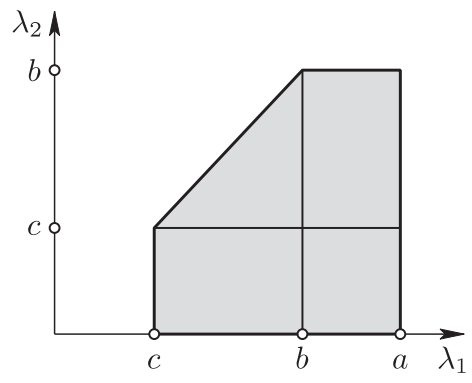

Рис. 18. Бифуркационное множество для биллиарда внутри эллипсоида в $\mathbb{E}^{3}$

Бифуркации торов Лиувилля для этой системы были исследованы в [41], [64], [65].

Разъясним подробно, как биллиардные движения соотносятся с диаграммой на рис. 18.

Над каждой точкой области, выделенной серым цветом, на диаграмме "висит" один или два трехмерных лиувиллевых тора. Отмеченные ребра соответствуют вырожденным множествам уровня.

Теперь детально опишем множества уровня над различными точками бифуркационного множества на рис. 18.

Гладкие листы. Над каждой внутренней точкой прямоугольников $[c, b] \times$ $[0, c],[b, a] \times[0, c]$ и треугольника с вершинами $(c, c),(b, c),(b, b)$ расположены два лиувиллевых тора $\mathbb{T}^{3}$. Над каждой внутренней точкой прямоугольника $[b, a] \times[c, b]$ “висит" ровно один тор $\mathbb{T}^{3}$. 
(a) Точки внутри $[c, b] \times[0, c]$ соответствуют движению, каустики которого - эллипсоид и однополостный гиперболоид. Один из лиувиллевых торов образован траекториями, обходящими ось $z$ в положительном направлении, а второй - траекториями, обходящими ее в отрицательном направлении.

(b) Точки внутри $[b, a] \times[0, c]$ соответствуют случаю, когда одна из каустик эллипсоид, а вторая - двуполостный гиперболоид. Каждый из лиувиллевых торов образован траекториями, обходящими ось $x$ в одном и том же направлении.

(c) Внутренние точки треугольника $(c, c)-(b, c)-(b, b)$ соответствуют движению, каустики которого - различные однополостные гиперболоиды. Каждый из лиувиллевых торов образован траекториями, обходящими ось $x$ в одном и том же направлении.

(d) Точки внутри прямоугольника $[b, a] \times[c, b]$ соответствуют случаю, когда обе каустики - гиперболоиды, но разных типов. Все соответствующие биллиардные траектории образуют один лиувиллев тор.

Сингулярные листы. Сингулярные листы лежат над ребрами диаграммы, представленной на рис. 18. Они возникают, когда хотя бы одна из каустик вырождена.

Поясним сначала, что такое вырожденная квадрика из софокусного семейства (2.10), а также геометрический смысл биллиардного движения с такой каустикой.

Вырожденные квадрики $\mathcal{Q}_{\lambda}, \lambda \in\{a, b, c\},-$ это следующие кривые, лежащие в координатных плоскостях:

$$
\begin{array}{lll}
\mathcal{Q}_{a}: & -\frac{y^{2}}{a-b}-\frac{z^{2}}{a-c}=1, & x=0, \\
\mathcal{Q}_{b}: & \frac{x^{2}}{a-b}-\frac{z^{2}}{b-c}=1, & y=0, \\
\mathcal{Q}_{c}: & \frac{x^{2}}{a-c}+\frac{y^{2}}{b-c}=1, & z=0 .
\end{array}
$$

Заметим, что $\mathcal{Q}_{c}$ - эллипс в плоскости $x y, \mathcal{Q}_{b}$ - гипербола в плоскости $x z$, а $\mathcal{Q}_{a}-$ в вещественном случае пустое множество. Однако мы будем трактовать $\mathcal{Q}_{a}$ как абстрактную кривую в плоскости $y z$. Вырожденные квадрики изображены на рис. 19. Если у биллиардного движения имеется одна из этих вырожденных каустик $\mathcal{Q}_{\lambda}$, то либо каждый отрезок траектории пересекает кривую $\mathcal{Q}_{\lambda}$, либо траектория целиком лежит в координатной плоскости, содержащей эту кривую. В последнем случае движение сводится к плоскому биллиарду внутри одного из эллипсов

$$
\begin{array}{lll}
\mathcal{E}_{a}: & \frac{y^{2}}{b}+\frac{z^{2}}{c}=1, & x=0, \\
\mathcal{E}_{b}: & \frac{x^{2}}{a}+\frac{z^{2}}{c}=1, & y=0, \\
\mathcal{E}_{c}: & \frac{x^{2}}{a}+\frac{y^{2}}{b}=1, & z=0 .
\end{array}
$$

Заметим, что при $\lambda=a$ имеются только плоские траектории. 
Каждый из этих трех эллипсов является пересечением $\mathcal{E}$ с одной из координатных гиперплоскостей (см. рис. 20).

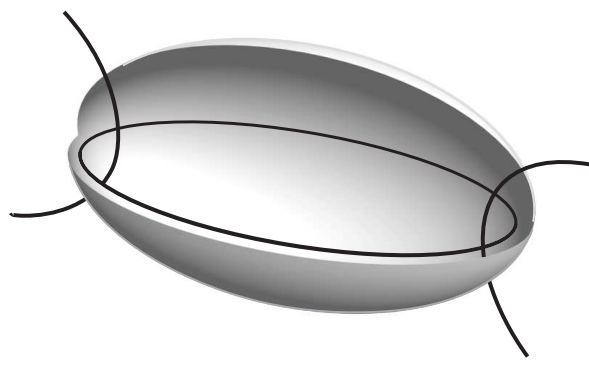

Рис. 19. Эллипсоид $\mathcal{E}$ и вырожденные квадрики $\mathcal{Q}_{b}, \mathcal{Q}_{c}$

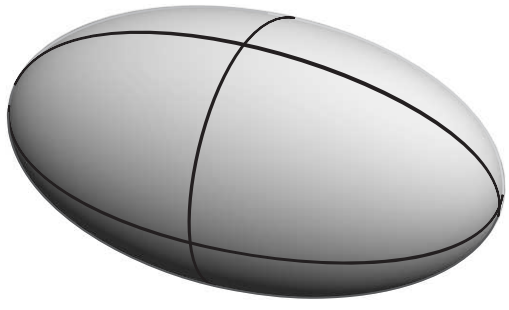

Рис. 20. Эллипсоид $\mathcal{E}$ и эллипсы $\mathcal{E}_{a}, \mathcal{E}_{b}, \mathcal{E}_{c}$

Помимо этих случаев вырожденных каустик, имеется еще один особый случай: одна из каустик совпадает с граничным эллипсоидом $\mathcal{E}$. Его можно рассматривать как предельный случай каустик $\mathcal{Q}_{\lambda}, \lambda \rightarrow 0_{+}$, а соответствующие траектории - геодезические на $\mathcal{E}$.

Теперь проанализируем ребра бифуркационной диаграммы на рис. 18.

Ребро $[c, a] \times\{0\}$. Как уже упоминалось, движение, соответствующее этому ребру, - геодезическое движение на $\mathcal{E}$. Таким образом, топология соответствующего подмножества изоэнергетической поверхности полностью описывается графом Фоменко на рис. 14.

Охарактеризуем этот случай:

(a) Точка $(c, 0)$ соответствует движению вдоль кривой $\mathcal{E}_{c}$. Два одномерных тора $\mathbb{T}^{1}$ лежат над точкой $(c, 0)$ бифуркационной диаграммы; каждый из них соответствует одному из возможных направлений потока.

(b) Внутренние точки отрезка $[c, b]$ на оси $\lambda_{1}$ соответствуют геодезическому движению на $\mathcal{E}$ с каустикой - однополостным гиперболоидом. Такие геодезические заполняют кольцо между двумя кривыми в пересечении $\mathcal{E}$ с гиперболоидом. Над каждой внутренней точкой отрезка висят два тоpa $\mathbb{T}^{2}$, соответствующие фиксированному направлению вращения вокруг оси $z$.

(c) Множество уровня, соответствующее $(b, 0)$, содержит две периодические траектории и четыре двумерные сепаратрисы. Периодические траектории проходят по кривой $\mathcal{E}_{b}$. Траектории на сепаратрисах проходят через омбилические точки эллипсоида.

(d) Внутренние точки отрезка $[b, a]$ оси $\lambda_{1}$ соответствуют геодезическому движению по $\mathcal{E}$ с каустикой - двуполостным гиперболоидом. Эти геодезические заполняют кольцо между двумя кривыми в пересечении $\mathcal{E}$ с гиперболоидом. Над каждой внутренней точкой отрезка висят два тора $\mathbb{T}^{2}$, каждый из которых соответствует некоторому направлению обхода оси $x$.

(е) Точка $(a, 0)$ соответствует движению вдоль $\mathcal{E}_{a}$. Над этой точкой находятся два одномерных тора $\mathbb{T}^{1}$; каждый из них соответствует одному из направлений движения. 
Ребро $\{a\} \times[0, b]$. Это ребро соответствует биллиардному движению с вырожденной каустикой $\mathcal{Q}_{a}$. Все траектории этого движения лежат в плоскости $y z$, так что на самом деле это ребро соответствует биллиарду в эллипсе. Топология множества, висящего над ребром, полностью описывается атомом Фоменко на рис. 6.

Ребро $[b, a] \times\{b\} . \quad$ Это ребро соответствует биллиардному движению с одной вырожденной каустикой $\mathcal{Q}_{b}$, в то время как вторая каустика - двуполостный гиперболоид.

\section{5. Интегрируемые возмущения потенциалом для эллиптического} биллиарда. В этом пункте мы изложим результаты по интегрируемым возмущениям потенциалом биллиардной системы в эллипсе. Анализ биллиардов с квадратичным потенциалом в эллипсоидах был проведен в [37].

Уравнение (см. [38]):

$$
\lambda V_{x y}+3\left(y V_{x}-x V_{y}\right)+\left(y^{2}-x^{2}\right) V_{x y}+x y\left(V_{x x}-V_{y y}\right)=0
$$

- частный случай уравнения Бертрана-Дарбу [66]-[68], описывающего необходимое и достаточное условие того, что в натуральной механической системе с двумя степенями свободы

$$
H=\frac{1}{2}\left(p_{x}^{2}+p_{y}^{2}\right)+V(x, y)
$$

переменные разделяются в эллиптических переменных или в некоторых координатах, являющихся вырожденным случаем эллиптических.

Решения уравнения (2.11) в виде полиномов Лорана по $x, y$ описаны в [34]. В [36] такие решения были естественным образом связаны с гипергеометрическими функциями Аппеля. Эта связь автоматически приводит к более широкому классу решений уравнения (2.11): новые потенциалы получаются при нецелых значениях параметров, что дает огромное семейство интегрируемых биллиардов с потенциалом внутри эллипса. Аналогичные формулы для возмущений потенциалом можно дать и в случае задачи Якоби о геодезических на эллипсоиде [34], [35]. Они указывают на связь между разделяющимися переменными в классических системах с одной стороны и теорией гипергеометрических функций с другой, однако эта связь до сих пор не очень понятна. Основными ссылками по функциям Аппеля являются работы [69]-[71].

Функция $F_{4}$ - это одна из четырех гипергеометрических функций двух переменных, введенных Аппелем (см. [69], [70]):

$$
F_{4}(a, b, c, d ; x, y)=\sum \frac{(a)_{m+n}(b)_{m+n}}{(c)_{m}(d)_{n}} \frac{x^{m}}{m !} \frac{y^{n}}{n !},
$$

где $(a)_{n}$ - обычный символ Похгаммера:

$$
(a)_{n}=\frac{\Gamma(a+n)}{\Gamma(a)}=a(a+1) \cdots(a+n-1), \quad(a)_{0}=1 .
$$

(Например, $\left.m !=(1)_{m} \cdot\right)$ 
Ряд (2.12) сходится при $\sqrt{x}+\sqrt{y} \leqslant 1$. Функции $F_{4}$ аналитически продолжаются до решений уравнений

$$
\begin{aligned}
x(1-x) & \frac{\partial^{2} F}{\partial x^{2}}-y^{2} \frac{\partial^{2} F}{\partial y^{2}}-2 x y \frac{\partial^{2} F}{\partial x \partial y} \\
& +[c-(a+b+1) x] \frac{\partial F}{\partial x}-(a+b+1) y \frac{\partial F}{\partial y}-a b F=0, \\
y(1-y) & \frac{\partial^{2} F}{\partial y^{2}}-x^{2} \frac{\partial^{2} F}{\partial x^{2}}-2 x y \frac{\partial^{2} F}{\partial x \partial y} \\
& +\left[c^{\prime}-(a+b+1) y\right] \frac{\partial F}{\partial y}-(a+b+1) x \frac{\partial F}{\partial x}-a b F=0 .
\end{aligned}
$$

2.5.1. Возмущения потенциалом для биллиарда внутри эллипса. Биллиардная система, описывающая частицу, свободно движущуюся в эллипсе

$$
\frac{x^{2}}{A}+\frac{y^{2}}{B}=1,
$$

вполне интегрируема и имеет дополнительный интеграл

$$
K_{1}=\frac{\dot{x}^{2}}{A}+\frac{\dot{y}^{2}}{B}-\frac{(\dot{x} y-\dot{y} x)^{2}}{A B}
$$

Нас интересуют возмущения потенциалом $V=V(x, y)$, при которых у возмущенной системы имеется интеграл $\widetilde{K}_{1}$ вида

$$
\widetilde{K}_{1}=K_{1}+k_{1}(x, y)
$$

где $k_{1}=k_{1}(x, y)$ зависит только от координат. Это специальное условие приводит к уравнению (2.11) на $V$ с $\lambda=A-B$.

В [34], [35] были приведены решения (2.11), имеющие вид многочленов Лорана. Для

$$
V_{\gamma}=\tilde{y}^{-\gamma}\left((1-\gamma) \tilde{x} F_{4}(1,2-\gamma, 2,1-\gamma ; \tilde{x}, \tilde{y})+1\right),
$$

где $\tilde{x}=x^{2} / \lambda, \tilde{y}=-y^{2} / \lambda$, в [36] был получен следующий более общий результат.

Теорема 2.29. Каждая функиия $V_{\gamma}$, определенная в $(2.13)$ с $\gamma \in \mathbb{C}$, является решением уравнения (2.11).

При нецелых $\gamma$ эта теорема дает новые примеры потенциалов. При целых $\gamma$ мы получаем лорановские решения.

2.5.2. Механическая интерпретация. Если $\gamma \in \mathbb{R}^{-}$и коэффициент при $V_{\gamma}$ положителен, то мы получаем потенциальный барьер вдоль оси $x$. Мы можем рассмотреть биллиард в верхней полуплоскости, тогда можно считать, что вдоль отрицательной части $y$-оси проведен разрез, так что потенциал превратился в однозначную вещественную функцию.

Решения уравнения (2.11) связаны также с интересными геометрическими вопросами. 
2.5.3. Биллиард в эллипсоиде с квадратичным потенциалом. В [37] рассмотрена биллиардная система в эллипсоиде

$$
\mathcal{E}: \quad\left(x, a^{-1} x\right)=1, \quad a=\left(a_{1}, \ldots, a_{n}\right), \quad 0<a_{1}<\cdots<a_{n},
$$

из $n$-мерного пространства, с квадратичным потенциалом вида

$$
\frac{\sigma\left(x_{1}^{2}+\cdots+x_{n}^{2}\right)}{2}, \quad \sigma=\text { const } .
$$

Этот потенциал называют также потенциалом Гука.

Пусть $\mathcal{B}:(x, v) \mapsto(\tilde{x}, \tilde{v})$ - биллиардное отображение, где $x, \tilde{x} \in \mathcal{E}$ - точки последовательных отражений от границы, а $v, \tilde{v}$ - векторы скорости в точках $x, \tilde{x}$ после отражения.

В следующей теореме приводятся явные формулы для $\mathcal{B}$.

ТЕОРема 2.30 [37]. Биллиардное отображение эквивалентно следующему отображению:

$$
\begin{aligned}
& \tilde{x}=-\frac{1}{\nu}\left[\left(\sigma-\left(v, a^{-1} v\right)\right) x+2\left(x, a^{-1} v\right) v\right], \\
& \tilde{v}=-\frac{1}{\nu}\left[\left(\sigma-\left(v, a^{-1} v\right) v\right) v-2 \sigma\left(x, a^{-1} v\right) x\right]+\mu a^{-1} \tilde{x}, \quad \mu=\frac{2\left(\tilde{v}, a^{-1} \tilde{x}\right)}{\left(\tilde{x}, a^{-2} \tilde{x}\right)}, \\
& \nu=\sqrt{4 \sigma\left(x, a^{-1} v\right)^{2}+\left(\sigma-\left(v, a^{-1} v\right)\right)^{2}} .
\end{aligned}
$$

Оказывается, что для биллиардного отображения $\mathcal{B}$ можно найти пары Лакса.

ПРЕДЛОЖЕНИЕ 2.31 [37]. С точностью до симметрии

$$
(x, v) \mapsto(-x,-v)
$$

биллиардное отображение $\mathcal{B}$ эквивалентно уравнению

$$
\widetilde{L}(\lambda)=M(\lambda) L(\lambda) M^{-1}(\lambda)
$$

əдe

$$
\begin{gathered}
L(\lambda)=\left(\begin{array}{cc}
q_{\lambda}(x, v) & q_{\lambda}(v, v)-\sigma \\
-q_{\lambda}(x, x)+1 & -q_{\lambda}(x, v)
\end{array}\right), \\
M(\lambda)=\left(\begin{array}{cc}
{\left[\sigma-\left(v, a^{-1} v\right)\right] \lambda+2\left(x, a^{-1} v\right) \mu} & 2 \sigma\left(x, a^{-1} v\right) \lambda-\left[\sigma-\left(v, a^{-1} v\right)\right] \mu \\
-2\left(x, a^{-1} v\right) \lambda & {\left[\sigma-\left(v, a^{-1} v\right)\right] \lambda}
\end{array}\right),
\end{gathered}
$$

а $\widetilde{L}(\lambda)$ зависит от $\tilde{x}, \tilde{v}$ в точности так же, как $L(\lambda)$ зависит от $x, v$.

Биллиард с потенциалом Гука имеет следующие геометрические свойства. 
ПРЕДЛОЖЕНИЕ 2.32 [37]. При $\sigma>0$ отрезки траекторий биллиарда с потенииалом Гука внутри эллипсоида $\mathcal{E}$ из $\mathbb{E}^{n}$ являются дугами эллипсов. Все эллипсы, содержащие отрезки одной траектории, касаются $n$ фиксированных квадрик, софокусных с $\mathcal{E}$. Параметры этих квадрик являются корнями характеристического полинома

$$
\left(\lambda-a_{1}\right) \cdots\left(\lambda-a_{n}\right) \operatorname{det} L(\lambda)
$$

Заметим, что число каустик движения с потенциалом Гука равно $n$, в то время как для невозмущенной системы это число по теореме Шаля равно $n-1$. Более того, как мы узнали от Ю. Фёдорова, число каустик равно $n+k-1$, если сепарабельное возмущение имеет степень $2 k$.

2.5.4. Задача Якоби о геодезических на эллипсоиде. У задачи Якоби о геодезических на эллипсоиде

$$
\frac{x^{2}}{A}+\frac{y^{2}}{B}+\frac{z^{2}}{C}=1
$$

имеется дополнительный интеграл

$$
K_{1}=\left(\frac{x^{2}}{A^{2}}+\frac{y^{2}}{B^{2}}+\frac{z^{2}}{C^{2}}\right)\left(\frac{\dot{x}^{2}}{A}+\frac{\dot{y}^{2}}{B}+\frac{\dot{z}^{2}}{C}\right) .
$$

Возмущения потенциалом $V=V(x, y, z)$, при которых у возмущенных систем есть интегралы вида

$$
\widetilde{K}_{1}=K_{1}+k(x, y, z),
$$

удовлетворяют системе уравнений

$$
\begin{gathered}
\left(\frac{x^{2}}{A^{2}}+\frac{y^{2}}{B^{2}}+\frac{z^{2}}{C^{2}}\right) V_{x y} \frac{A-B}{A B}-3 \frac{y V_{x}}{B^{2} A}+3 \frac{x V_{y}}{A^{2} B} \\
+\left(\frac{x^{2}}{A^{3}}-\frac{y^{2}}{B^{3}}\right) V_{x y}+\frac{x y}{A B}\left(\frac{V_{y y}}{A}-\frac{V_{x x}}{B}\right)+\frac{z x V_{z y}}{C A^{2}}-\frac{z y V_{z x}}{C B^{2}}=0, \\
\left(\frac{x^{2}}{A^{2}}+\frac{y^{2}}{B^{2}}+\frac{z^{2}}{C^{2}}\right) V_{y z} \frac{B-C}{B C}-3 \frac{z V_{y}}{C^{2} B}+3 \frac{y V_{z}}{B^{2} C} \\
+\left(\frac{y^{2}}{B^{3}}-\frac{z^{2}}{C^{3}}\right) V_{y z}+\frac{y z}{B C}\left(\frac{V_{z z}}{B}-\frac{V_{y y}}{C}\right)+\frac{x y V_{x z}}{A B^{2}}-\frac{x z V_{x y}}{A C^{2}}=0, \\
\left(\frac{x^{2}}{A^{2}}+\frac{y^{2}}{B^{2}}+\frac{z^{2}}{C^{2}}\right) V_{z x} \frac{C-A}{A C}-3 \frac{x V_{z}}{A^{2} C}+3 \frac{z V_{x}}{C^{2} A} \\
+\left(\frac{z^{2}}{C^{3}}-\frac{x^{2}}{A^{3}}\right) V_{z x}+\frac{x z}{A C}\left(\frac{V_{x x}}{C}-\frac{V_{z z}}{A}\right)+\frac{z y V_{x y}}{B C^{2}}-\frac{y x V_{y z}}{B A^{2}}=0 .
\end{gathered}
$$

Это аналог уравнения Бертрана-Дарбу (2.11) (см. [36]).

Пусть

$$
\frac{x^{2} C(A-C)}{z^{2}(B-A) A}=\hat{x}, \quad \frac{y^{2} C(C-B)}{z^{2}(B-A) B}=\hat{y} .
$$

Следующее утверждение также было доказано в [36]. 
Tеорема 2.33. Для всякого $\gamma \in \mathbb{C}$ функиия

$$
V_{\gamma}=(-\gamma+1)\left(\frac{z^{2}}{x^{2}}\right)^{\gamma} F_{4}(1,-\gamma+2,2,-\gamma+1 ; \hat{x}, \hat{y})
$$

является решением системы (2.14).

Таким образом, решая уравнение Бертрана-Дарбу и его обобщения, как это делалось, например, в теоремах 2.29 и 2.33, мы получаем большие семейства механических систем с двумя степенями свободы, в которых переменные разделяются. Хорошо известно, что системы с двумя степенями свободы и разделяющимися переменными необходимо являются системами типа Лиувилля (см. [68]).

Теперь возникает естественный вопрос о теореме типа Понселе, описывающей периодические решения таких возмущенных систем. Оказывается, что Дарбу исследовал и этот вопрос: в [44] он изучал обобщения теоремы Понселе на случай поверхностей Лиувилля.

\section{3. Закон биллиардного отражения и гиперэллиптические кривые}

В этом разделе мы продемонстрируем наличие глубоких и тесных связей между общими гиперэллиптическими якобианами и интегрируемыми биллиардными системами, порожденными пучками квадрик.

В п. 3.1 мы приводим определение биллиардного закона отражения в проективной геометрии, используя вместо метрик семейства софокусных квадрик. Затем мы перейдем к важным теоремам об отражении и о двойном отражении из [21], к понятию виртуального отражения из [44] и к нескольким важным следствиями из [29]. В п. 3.2 вводится обобщенная кривая Кэли. В п. 3.3 мы приводим конструкцию биллиардной алгебры на якобиане этой гиперэллиптической кривой. В п. 3.4 вводится понятие $s$-слабых траекторий Понселе и находятся соответствующие условия типа Кэли. Результаты, представленные в пп. 3.5 и 3.6, свидетельствуют о возможностях метода биллиардных алгебр: в их числе обобщения пространственной теоремы Гриффитса-Харриса, теоремы Вейра и теоремы Дарбу. Изложение в пп. 3.3-3.6 опирается на [39].

3.1. Динамика биллиардов в проективной постановке. В этом пункте рассматривается $d$-мерное проективное пространство над произвольным полем характеристики отличной от 2 .

Сначала нам потребуется проективное определение биллиардного отражения, не использующее метрик. В этом мы следуем [21].

Пусть $\mathcal{Q}_{1}$ и $\mathcal{Q}_{2}-$ квадрики. Обозначим через $u$ касательную плоскость $\mathcal{Q}_{1}$ в точке $x$, а через $z$ полюс $u$ относительно $\mathcal{Q}_{2}$. Пусть прямые $\ell_{1}$ и $\ell_{2}$ пересекаются в $x$, и пусть плоскость, содержащая их, пересекает $u$ по прямой $\ell$.

ОПРЕДЕЛЕНИЕ 3.1. Если прямые $\ell_{1}, \ell_{2}, x z, \ell$ компланарны и гармонически сопряжены, то скажем, что лучи $\ell_{1}$ и $\ell_{2}$ удовлетворяют закону отражения в точке $x$ квадрики $\mathcal{Q}_{1}$ относительно софокусной системы, содержащей $\mathcal{Q}_{1}$ и $\mathcal{Q}_{2}$.

После введения системы координат, в которой квадрики $\mathcal{Q}_{1}$ и $\mathcal{Q}_{2}$ софокусны в обычном смысле, так определенное отражение перейдет в обычное. 
ТЕОРема 3.2 (теорема об отражении). Пусть лучи $\ell_{1}$ и $\ell_{2}$ удовлетворяют закону отражения в точке $x$ квадрики $\mathcal{Q}_{1}$ относительно софокусной системы, задаваемой квадриками $\mathcal{Q}_{1}$ u $\mathcal{Q}_{2}$. Допустим, что $\ell_{1}$ пересекает $\mathcal{Q}_{2}$ в $y_{1}^{\prime}$ и $y_{1}$, пусть $и$ - касательная плоскость $\kappa \mathcal{Q}_{1}$ в $x$, а z -ее полюс относительно $\mathcal{Q}_{2}$. Тогда прямые $y_{1}^{\prime} z$ и $y_{1} z$ содержат точки $y_{2}^{\prime}$ и $y_{2}$ пересечения луча $\ell_{2} c \mathcal{Q}_{2}$. Обратное утверждение тоже верно.

СлЕДСТВИЕ 3.3. Предположим, что лучи $\ell_{1} u \ell_{2}$ удовлетворяют закону отражения от $\mathcal{Q}_{1}$ относительно софокусной системы, задаваемой квадриками $\mathcal{Q}_{1}$ и $\mathcal{Q}_{2}$. Тогда $\ell_{1}$ касается $\mathcal{Q}_{2}$ в том и только том случае, когда $\ell_{2}$ касается $\mathcal{Q}_{2} ; \ell_{1}$ пересекает $\mathcal{Q}_{2}$ в двух точках в том и только том случае, когда $\ell_{2}$ пересекает $\mathcal{Q}_{2}$ в двух точках.

Следующее утверждение является одним из основных инструментов проективного анализа биллиардной динамики.

ТЕОРема 3.4 (теорема о двойном отражении). Для заданных квадрик $\mathcal{Q}_{1}, \mathcal{Q}_{2}$ пусть $\ell_{1}$ - прямая, пересекаюшая $\mathcal{Q}_{1}$ в точке $x_{1}$ и $\mathcal{Q}_{2}$ в $y_{1}$. Пусть $u_{1}, v_{1}$ касательные плоскости $\kappa \mathcal{Q}_{1}, \mathcal{Q}_{2}$ в точках $x_{1}, y_{1}$, и пусть $z_{1}, w_{1}-$ их полюсы относительно $\mathcal{Q}_{2}$ и $\mathcal{Q}_{1}$. Обозначим через $x_{2}$ вторую точку пересечения прямой $w_{1} x_{1} c \mathcal{Q}_{1}$, через $y_{2}$ точку пересечения $y_{1} z_{1} c Q_{2}$ и через $\ell_{2}, \ell_{1}^{\prime}, \ell_{2}^{\prime}$ прямые $x_{1} y_{2}, y_{1} x_{2}, x_{2} y_{2}$. Тогда пары $\ell_{1}, \ell_{2} ; \ell_{1}, \ell_{1}^{\prime} ; \ell_{2}, \ell_{2}^{\prime} ; \ell_{1}^{\prime}, \ell_{2}^{\prime}$ удовлетворяют закону

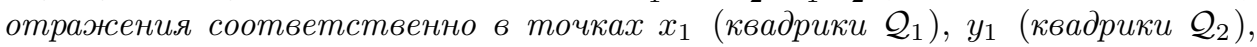
$y_{2}\left(\kappa в а д р и к и ~ \mathcal{Q}_{2}\right)$ и $x_{2}\left(\kappa в а д р и к и ~ \mathcal{Q}_{1}\right)$.

СлЕДСТВиЕ 3.5. Если прямая $\ell_{1}$ касается квадрики $\mathcal{Q}^{\prime}$, софокусной с $\mathcal{Q}_{1}$ и $\mathcal{Q}_{2}$, то лучи $\ell_{2}, \ell_{1}^{\prime}$ и $\ell_{2}^{\prime}$ также касаются $\mathcal{Q}^{\prime}$.

3.1.1. Виртуальные биллиардные траектории. Помимо реального движения биллиардного шара в $\mathbb{E}^{d}$, интерес представляют виртуальные отражения. Дарбу рассматривал их в [44] (см. гл. XIV книги IV в т. 2). В этом пункте мы рассматриваем некоторые результаты из [29], [39], в которых устанавливается и обобщается свойство виртуального отражения, сформулированное Дарбу для трехмерного случая (см. сноску [44; с. 320-321]).

Формальным образом виртуальное отражение от квадрики $\mathcal{Q}$ в евклидовом пространстве определяется как отображение луча $\ell$ с концом в $P_{0}\left(P_{0} \in \mathcal{Q}\right)$ в луч, дополнительный к образу $\ell$ относительно обычного отражения от $\mathcal{Q}$ в точке $P_{0}$.

Заметим, что в случае обычных отражений ровно одна эллиптическая координата имеет локальный экстремум в точке отражения, а именно, та, что соответствует квадрике $\mathcal{Q}$. С другой стороны, на виртуальном отраженном луче только у этой координаты нет локального экстремума в точке отражения. В двумерном случае виртуальное отражение легко определить как отражение от другой софокусной квадрики, проходящей через $P_{0}$. В более высоких размерностях виртуальное отражение можно рассматривать как реальное отражение от прямой, нормальной к $\mathcal{Q}$ в $P_{0}$.

Понятия реального и виртуального отражения не могут быть прямо обобщены на проективное пространство, так как здесь мы существенно используем естественный порядок на поле вещественных чисел. Однако можно ввести некоторую конфигурацию, связанную с реальным и виртуальным отражением, так, чтобы ее свойства сохранялись и в проективном случае. 
Пусть $X_{1}, X_{2}$ и $Y_{1}, Y_{2}$ - соответственно точки квадрик $\mathcal{Q}_{1}$ и $\mathcal{Q}_{2}$ из $\mathbb{P}^{d}$.

ОПРЕДЕЛЕНИЕ 3.6. Скажем, что четверка точек $X_{1}, X_{2}, Y_{1}, Y_{2}$ задает конфигуращию виртуального отражения, если пары прямых $X_{1} Y_{1}, X_{1} Y_{2} ; X_{2} Y_{1}$, $X_{2} Y_{2} ; X_{1} Y_{1}, X_{2} Y_{1} ; X_{1} Y_{2}, X_{2} Y_{2}$ удовлетворяют закону отражения в точках $X_{1}, X_{2}$ квадрики $\mathcal{Q}_{1}$ и в точках $Y_{1}, Y_{2}$ квадрики $\mathcal{Q}_{2}$ относительно софокусной системы, задаваемой $\mathcal{Q}_{1}$ и $\mathcal{Q}_{2}$.

Если к тому же касательные плоскости к $\mathcal{Q}_{1}, \mathcal{Q}_{2}$ в $X_{1}, X_{2}$ и $Y_{1}, Y_{2}$ входят в один пучок, то скажем, что эти точки задают конфигурацию двойного отражения.

Утверждение Дарбу теперь можно обобщить и доказать следующим образом.

ТеОРема 3.7 [29]. Пусть $\mathcal{Q}_{1}, \mathcal{Q}_{2}$ - квадрики в проективном пространстве $\mathbb{P}^{d}, X_{1}, X_{2}$ - точки $\mathcal{Q}_{1}$ и $Y_{1}, Y_{2}$ - точки $\mathcal{Q}_{2}$. Если касательнье плоскости $\kappa$ квадрикам в этих точках входят в один пучок, то $X_{1}, X_{2}, Y_{1}, Y_{2}$ задают конфигурачию виртуалвного отражения.

Далее, пусть проективное пространство определено над полем вещественньх чисел. Введем систему координат, в которой $\mathcal{Q}_{1}$ и $\mathcal{Q}_{2}$ оказываются софокусными эллипсоидами в евклидовом пространстве. Если $\mathcal{Q}_{1}$ лежит внутри $\mathcal{Q}_{2}$, то стороны четырехугольника $X_{1} Y_{1} X_{2} Y_{2}$ связаны реальным отражением от $\mathcal{Q}_{2}$ и виртуальным отражением от $\mathcal{Q}_{1}$.

ДокАЗАтельство. Пусть $\xi_{1}, \xi_{2}$ и $\eta_{1}, \eta_{2}$ - касательные плоскости соответственно к $\mathcal{Q}_{\lambda_{1}}$ в точках $X_{1}, X_{2}$ и к $\mathcal{Q}_{\lambda_{2}}$ в $Y_{1}, Y_{2}$. Все эти гиперплоскости входят в пучок, так что их полюсы относительно любой квадрики коллинеарны; в частности, полюс $P$ гиперплоскости $\xi_{1}$ лежит на прямой $Y_{1} Y_{2}$. Если $Q=Y_{1} Y_{2} \cap \xi_{1}$, то пары $P, Q$ и $Y_{1}, Y_{2}$ гармонически сопряжены. Следовательно, прямые $X_{1} Y_{1}$ и $X_{1} Y_{2}$ удовлетворяют закону отражения в $\xi_{1}$. Завершение доказательства проходит аналогично.

Приведем также утверждение, обратное к этой теореме.

ПРЕДЛОЖЕНИЕ 3.8 [29]. Пусть $X_{1}, X_{2}$ u $Y_{1}, Y_{2}$ - пары точек на софокусных эллипсоидах $\mathcal{Q}_{1}$ и $\mathcal{Q}_{2}$ в евклидовом пространстве $\mathbb{E}^{d}$, и пусть $\alpha_{1}, \alpha_{2}, \beta_{1}, \beta_{2}-$ соответствующие касательные плоскости. Если четверка $X_{1}, X_{2}, Y_{1}, Y_{2}$ образует конфигурацию виртуального отражения, то плоскости $\alpha_{1}, \alpha_{2}, \beta_{1}, \beta_{2}$ входят в один пучок.

3.1.2. Обобщения основной теоремы Лебега на более высокие размерности. Мы завершим п. 3.1 анализом обобщений впечатляющей конструкции Лебега из [15] на случай более высоких размерностей. Представленные результаты были получены в [29].

У центральной леммы из [15] имеется следующий аналог в больших размерностях.

Лемма 3.9 [29]. Пусть $\mathcal{Q}_{1}, \mathcal{Q}_{2}-$ квадрики из софокусной системъ, и пусть прямые $\ell_{1}, \ell_{2}$ удовлетворяют закону отражения в точке $X_{1}$ квадрики $\mathcal{Q}_{1}$, а $\ell_{2}, \ell_{3}$ удовлетворяют ему в точке $Y_{2}$ квадрики $\mathcal{Q}_{2}$. Тогда прямая $\ell_{1}$ пересекает $\mathcal{Q}_{2}$ в такой точке $Y_{1}$, a $\ell_{3}$ пересекает $\mathcal{Q}_{1}$ в такой точке $X_{2}$, что пары прямых $\ell_{1}, Y_{1} X_{2}$ и $Y_{1} X_{2}, \ell_{3}$ удовлетворяют закону отражения соот- 
ветственно в точках $Y_{1} u X_{2}$ квадрик $\mathcal{Q}_{2} u \mathcal{Q}_{1}$. Более того, касательные плоскости в $X_{1}, X_{2}, Y_{1}, Y_{2}$ к этим квадрикам входят в один пучок.

Этот результат можно доказать непосредственным применением теоремы 3.7 о виртуальных отражениях. Однако между леммой 3.9 и соответствующим результатом на плоскости нет полной аналогии. В общем положении прямые $\ell_{1}$, $\ell_{3}$ и $\ell_{2}, Y_{1} Y_{2}$ скрещиваются, так что у нас нет третьей пары плоскостей, касающихся квадрики и содержащих точки пересечения указанных двух пар прямых.

Все же мы можем сформулировать полный аналог основной леммы.

ТЕОРема 3.10 [29]. Пусть $\mathcal{F}$ - двойственный пучок квадрик в трехмерном пространстве.

Для любой квадрики $\Gamma_{0} \in \mathcal{F}$ существуют четыре плоскости $\alpha, \beta, \gamma, \delta$, касательные $\kappa \Gamma_{0}$, и такие квадрики $\Gamma_{1}, \Gamma_{2}, \Gamma_{3} \in \mathcal{F}$, соприкасающиеся соответственно с парами пересекающихся прямых $\alpha \beta u \gamma \delta, \alpha \gamma u \beta \delta, \alpha \delta$ и $\beta \gamma$, что все касательные плоскости $\kappa \Gamma_{1}, \Gamma_{2}, \Gamma_{3}$ в точках касания с прямыми входят в один пучок $\Delta$. Более того, шесть пересекающихся прямых входят в одну связку.

3.2. Обобщенная кривая Кэли. Продолжим исследования, начатые в п. 2.1. Пусть дано софокусное семейство квадрик в $d$-мерном пространстве. Тогда каждая прямая в пространстве определяет некоторую замечательную риманову поверхность.

ОПРедЕЛЕНиЕ 3.11. Пусть $\ell$ - прямая, не лежащая на квадрике из фиксированного софокусного семейства в проективном пространстве $\mathbb{P}^{d}$. Обобщенная кривая Кэли $\mathcal{C}_{\ell}-$ это многообразие касательных плоскостей к квадрикам из софокусного семейства в точках $\ell$.

Эта кривая вложена естественным образом в двойственное пространство $\mathbb{P}^{d *}$.

На рис. 21 мы видим плоскости, соответствующие одной точке прямой $\ell$ в трехмерном пространстве.

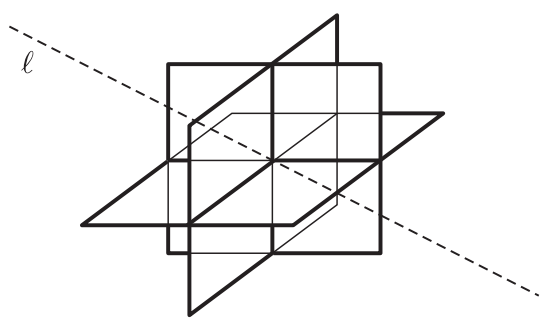

Рис. 21. Три точки обобщенной кривой Кэли в размерности 3

ПРЕДЛОЖЕНИЕ 3.12. Обобщенная кривая Кэли - это гиперэллиптическая кривая рода $g=d-1$ при $d \geqslant 3$. Ее естественная реализация в $\mathbb{P}^{d *}$ имеет степень $2 d-1$. 
Естественная инволюция $\tau_{\ell}$ обобщенной кривой Кэли $\mathcal{C}_{\ell}$ переводит касательные плоскости в точках пересечения $\ell$ с каждой квадрикой из софокусного семейства одна в другую. Легко видеть, что неподвижные точки этой инволюции соответствуют $d-1$ квадрике, касающейся $\ell$ и $d+1$ вырожденной квадрике софокусного семейства.

Уравнение обобщенной кривой Кэли, соответствующей софокусному семейству вида (2.1), можно записать в виде

$$
y^{2}=\mathcal{P}(x),
$$

где $\mathcal{P}(x)$ - полином $(2.2)$.

Важно отметить, что постоянные $\alpha_{1}, \ldots, \alpha_{d-1}$, соответствующие квадрикам, соприкасающимся с $\ell$, могут принимать не любые значения. Точнее, вслед за [64], [41] мы можем сформулировать следующий результат.

ПредлОЖениЕ 3.13. Прямая в $\mathbb{E}^{d}$, касающаяся $d-1$ различных невырожденных квадрик $\mathcal{Q}_{\alpha_{1}}, \ldots, \mathcal{Q}_{\alpha_{d-1}}$ из семейства $(2.1)$, существует тогда и только тогда, когда множество $\left\{a_{1}, \ldots, a_{d}, \alpha_{1}, \ldots, \alpha_{d-1}\right\}$ можно упорядочить в виде $b_{1}<b_{2}<\cdots<b_{2 d-1}$, где $\alpha_{j} \in\left\{b_{2 j-1}, b_{2 j}\right\} \quad(1 \leqslant j \leqslant d-1)$.

Как было замечено в [29], обобщенная кривая Кэли изоморфна изоспектральной кривой Веселова-Мозера.

Эта кривая также изоморфна естественным образом кривым, изученным Кнёррером, Донаги и Райдом (RDK-кривые). Следующий пункт мы начнем с построения этого изоморфизма, с тем чтобы установить связь между законом биллиардного отражения и алгебраической структурой якобиана $\operatorname{Jac}\left(\mathcal{C}_{\ell}\right)$.

\section{3. Закон биллиардного отражения и алгебраическая структура} на многообразии $\mathcal{A}_{\ell}$. Целью этого пункта является введение на $\mathcal{A}_{\ell}$ алгебраической структуры, естественно связанной с биллиардными движениями. Сначала мы покажем, что обобщенная кривая Кэли изоморфна RDK-кривой. Затем для лучшего понимания и наглядности мы подробно опишем биллиардную алгебру и докажем несколько ее красивых геометрических свойств в случае размерности 3 , т. е. когда соответствующая кривая имеет род 2 . За этим последует общая конструкция.

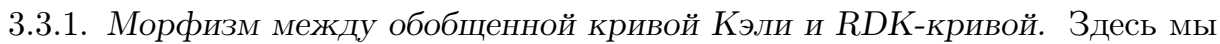
установим связь между обобщенной кривой Кэли, определенной выше, и кривыми, изученными Кнёррером, Донаги и Райдом и проследим за связями между биллиардными конструкциями и алгебраической структурой соответствующих абелевых многообразий.

Пусть $\ell$ - прямая в $\mathbb{E}^{d}$, касающаяся квадрик $\mathcal{Q}_{\alpha_{1}}, \ldots, \mathcal{Q}_{\alpha_{d-1}}$ из софокусного семейства (2.1). Обозначим через $\mathcal{A}_{\ell}$ семейство всех прямых, касающихся тех же $d-1$ квадрик. Заметим, что в соответствии со следствием из теоремы об отражении множество $\mathcal{A}_{\ell}$ инвариантно относительно биллиардного отражения от любой из софокусных квадрик.

Начнем с простого замечания. 
Лемма 3.14. Пусть прямые $\ell$ u $\ell^{\prime}$ удовлетворяют закону отражения $в$ точке z квадрики $\mathcal{Q}$, предположим, что они касаются софокусной квадрики $\mathcal{Q}_{1}$ в точках $z_{1}$ и $z_{2}$. Тогда пересечение касательных пространств $T_{z_{1}} \mathcal{Q}_{1} \cap T_{z_{2}} \mathcal{Q}_{1}$ лежит в касательном пространстве $T_{z} \mathcal{Q}$.

ДокАЗАтельство. Результат следует из теоремы об отражении: поскольку полюсы $z_{1}, z_{2}$ и $w$ плоскостей $T_{z_{1}} \mathcal{Q}_{1}, T_{z_{2}} \mathcal{Q}_{1}$ и $T_{z} \mathcal{Q}$ относительно квадрики $\mathcal{Q}_{1}$ коллинеарны, плоскости лежат в одном пучке.

Следуя [41], для $d-1$ аффинных квадрик $\mathcal{Q}_{\alpha_{1}}, \ldots, \mathcal{Q}_{\alpha_{d-1}}$ мы можем рассмотреть их проективные замыкания $\mathcal{Q}_{\alpha_{1}}^{p}, \ldots, \mathcal{Q}_{\alpha_{d-1}}^{p}$ и пересечение $V$ двух квадрик из $\mathbb{P}^{2 d-1}$ :

$$
\begin{gathered}
x_{1}^{2}+\cdots+x_{d}^{2}-y_{1}^{2}-\cdots-y_{d-1}^{2}=0, \\
a_{1} x_{1}^{2}+\cdots+a_{d} x_{d}^{2}-\alpha_{1} y_{1}^{2}-\cdots-\alpha_{d-1} y_{d-1}^{2}=x_{0}^{2} .
\end{gathered}
$$

Через $F=F(V)$ обозначим множество $(d-2)$-мерных линейных подпространств $V$. Для данного $L \in F$ обозначим через $F_{L}$ замыкание в $F$ множества $\left\{L^{\prime} \in F \mid \operatorname{dim} L \cap L^{\prime}=d-3\right\}$. Как показано в [40], $F_{L}$ - неособая гиперэллиптическая кривая рода $d-1$. Заметим, что при $d=3$, т. е. когда кривая $\mathcal{C}_{\ell}$ имеет род 2 , изоморфизм между $F(V)$ и якобианом гиперэллиптической кривой был установлен в [72].

Проекция

$$
\pi: \mathbb{P}^{2 d-1} \backslash\{(x, y): x=0\} \rightarrow \mathbb{P}^{d}, \quad \pi^{\prime}(x, y)=x,
$$

отображает $L \in F(V)$ в подпространство $\pi^{\prime}(L) \subset \mathbb{P}^{d}$ коразмерности $2, \pi^{\prime}(L)$ касается квадрик $\mathcal{Q}_{\alpha_{1}}^{p *}, \ldots, \mathcal{Q}_{\alpha_{d-1}}^{p *}$, двойственных к $\mathcal{Q}_{\alpha_{1}}^{p}, \ldots, \mathcal{Q}_{\alpha_{d-1}}^{p}$.

Таким образом, двойственное к $\pi^{\prime}(L)$ пространство $\pi^{*}(L)$ является прямой, касающейся квадрик $\mathcal{Q}_{\alpha_{1}}^{p}, \ldots, \mathcal{Q}_{\alpha_{d-1}}^{p}$.

Для обобщенной кривой Кэли $\mathcal{C}_{\ell}$, определенной как семейство касательных гиперплоскостей, мы можем дать и другую интерпретацию - как множества прямых из $\mathcal{A}_{\ell}$, пересекающих $\ell$. А именно, для почти каждой касательной гиперплоскости имеется единственная прямая $\ell^{\prime}$, полученная из $\ell$ биллиардным отражением. Имея в виду эту идентификацию, легко доказать следующий результат.

СлеДСТВИЕ 3.15. Существует бирациональный морфизм между обобщенной кривой Кэли $\mathcal{C}_{\ell}$ и кривой Райда-Донаги-Кнёррера $F_{L}$ с $L=\pi^{*-1}(\ell)$, задаваемый формулой

$$
j: \ell^{\prime} \mapsto L^{\prime}, \quad L^{\prime}=\pi^{*-1}\left(\ell^{\prime}\right),
$$

где $\ell^{\prime}$ - прямая, полученная из $\ell$ биллиардным отражением от софокусной квадрики.

ДокАЗАТЕЛЬСтво. Это следствие предыдущих лемм, а также леммы 4.1 и следствия 4.2 в [41].

Итак, лемма 3.14 связывает динамику биллиардов в эллипсоидах с алгебраической структурой некоторых абелевых многообразий. Эту связь можно использовать двояко: как для приложения алгебраических методов к анализу биллиардных движений, так и, наоборот, используя биллиардные конструкции для более эффективного, конструктивного и наглядного анализа алгебраической структуры. 
В следующем пункте мы используем эту связь в конструкции биллиардной алгебры, задающей на $\mathcal{A}_{\ell}$ структуру группы.

3.3.2. Случай рода 2. Прежде чем перейти к общему случаю, мы хотим особо выделить биллиардные конструкции, используемые в первом нетривиальном случае, случае рода 2.

Основной принцип, определение и первые свойства операции. Сформулируем основной приниип:

Во всякой конфигурации виртуального отражения сумма прямъх равна нулю, если четыре касательных плоскости в точках отражения входят в один пучок.

Напомним, что, согласно определению 3.6, такую конфигурацию четырех прямых, задающих конфигурацию виртуального отражения, в которой касательные плоскости принадлежат одному пучку, мы будем называть конфигурацией двойного отражения.

Нулевой элемент. Зафиксируем прямую $\mathcal{O} \in \mathcal{A}_{\ell}$.

Противоположный элемент. Сначала положим по определению $-\mathcal{O}:=\mathcal{O}$. Для прямой $x \in \mathcal{C}_{\mathcal{O}}$ положим

$$
-x:=\tau_{\mathcal{O}}(x),
$$

где $\tau_{\mathcal{O}}$ - гиперэллиптическая инволюция кривой $\mathcal{C}_{\mathcal{O}}$.

ПреДЛОЖЕНИЕ 3.16. Для всякой точки $x \in \mathcal{C}_{\mathcal{O}}$ nрямые $x$ u $-x$ получаются из $\mathcal{O}$ отражением от одной и той же квадрики $\mathcal{Q}_{x}$ из софокусного семейства.

Кроме того, пусть $\pi$ - единственная плоскость из пучка, задаваемого касательными плоскостями $\kappa \mathcal{Q}_{x}$ в точках пересечения с $\mathcal{O}$, такая, что $\pi$ ортогональна $\mathcal{O}$, и пусть $\mathcal{Q}_{\mathcal{O}}$ - единственная квадрика из собокусного семейства, которой $\pi$ касается. Тогда точка пересечения $\pi$ и $\mathcal{O}$ лежит в $\mathcal{Q}_{\mathcal{O}}$.

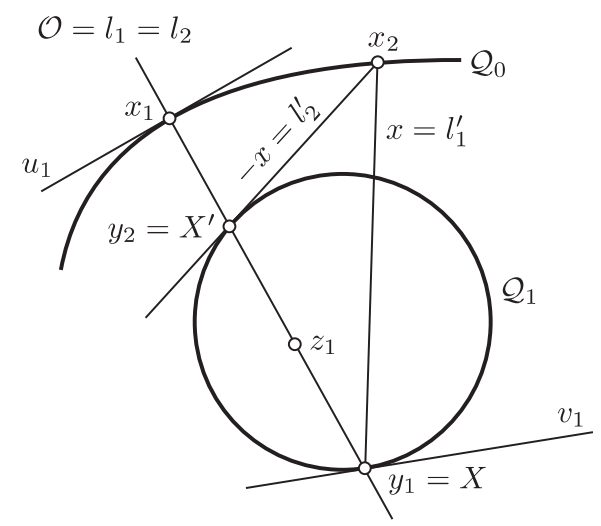

Рис. 22. Предложение 3.16

ДокАЗАТЕЛЬство. Это следует из вырожденного случая теоремы о двойном отражении, примененного к квадрикам $\mathcal{Q}_{x}$ и $\mathcal{Q}_{\mathcal{O}}, \mathcal{O}=l_{1}=l_{2}$. См. рис. 22.

СлЕДСТвИЕ 3.17. Плоскость $\pi$ и квадрика $\mathcal{Q}_{\mathcal{O}}$ не зависят от $x$. Прямая $\mathcal{O}$ при отражении от $\mathcal{Q}_{\mathcal{O}}$ переходит в себя. 
Теперь пусть $x \in \mathcal{A}_{\ell}$, но $x$ не лежит в $\mathcal{C}_{\mathcal{O}}$, так что $x$ не пересекает $\mathcal{O}$ и эти две прямые порождают проективное пространство прямых. Это пространство пересекает $\mathcal{A}_{\ell}$ по дивизору

$$
\mathcal{O}+x+p+q
$$

(см., например, [73], [74]).

Из теоремы о двойном отражении можно видеть, что прямые $\mathcal{O}, x, p$ и $q$ образуют конфигурацию двойного отражения. Определим прямую $-x$ так, чтобы она задавала конфигурацию двойного отражения с $\mathcal{O},-p,-q$.

Из определения непосредственно видно, что $-(-x)=x$ для всякого $x \in \mathcal{A}_{\ell}$.

Следующее свойство вытекает из предложения 3.16 и теоремы о двойном отражении.

ПрЕДЛОЖЕНИЕ 3.18. Прямые $x u-x$ пересекаются и удовлетворяют закону отражения от $\mathcal{Q}_{\mathcal{O}}$.

Следующий пример иллюстрирует только что проведенное построение.

ПримеР 3.19. Пусть прямая $\mathcal{O}$ ортогональна одной из координатных гиперплоскостей. Тогда $\mathcal{Q}_{\mathcal{O}}=\pi$ совпадает с этой гиперплоскостью, и к тому же среди каустик $\mathcal{Q}_{1}, \ldots, \mathcal{Q}_{d-1}$ не может быть двух квадрик одного типа.

Согласно [64], как раз тот случай, когда все квадрики имеют различные типы, отвечает ситуации, когда $\mathcal{A}_{\ell}$ состоит из единственной вещественной связной компоненты, изоморфной компоненте связности, $\operatorname{Jac}\left(\mathcal{C}_{\mathcal{O}}\right)(\mathbb{R})$, содержащей нуль.

В этом случае для всякой прямой $x \in \mathcal{A}_{\ell}$ противоположный элемент $-x$ определяется как прямая, симметричная $x$ относительно плоскости $\mathcal{Q}_{\mathcal{O}}$.

Сложение. Определим операцию

$$
+: \mathcal{A}_{\ell} \times \mathcal{A}_{\ell} \rightarrow \mathcal{A}_{\ell}
$$

Положим $\mathcal{O}+x=x+\mathcal{O}=x$ для всех $x \in \mathcal{A}_{\ell}$.

При $s_{1}, s_{2} \in \mathcal{C}_{\mathcal{O}}$ определим $s_{1}+s_{2}$ как прямую, образующую конфигурацию двойного отражения с $-s_{1},-s_{2}, \mathcal{O}$. Очевидно, что $s_{1}+s_{2}=s_{2}+s_{1}$.

Заметим, что $-s_{1},-s_{2}$ - единственные прямые из $\mathcal{A}_{\ell}$, пересекающие как $s_{1}+s_{2}$, так и $\mathcal{O}$ (см. [73]). Таким образом, имеет место следующее утверждение.

ЛЕмма 3.20. Всякую прямую $x \in \mathcal{A}_{\ell} \backslash \mathcal{C}_{\mathcal{O}}$ можно единственным образом представить как сумму двух прямых, пересекающих $\mathcal{O}$.

Теперь пусть $s \in \mathcal{C}_{\mathcal{O}}, x \in \mathcal{A}_{\ell} \backslash \mathcal{C}_{\mathcal{O}}$ (см. рис. 23). В соответствии со сказанным выше, рассмотрим такие прямые $p, q \in \mathcal{C}_{\mathcal{O}}$, что $x=p+q$, и построим

$$
p_{1}=(-s)+(-p), \quad q_{1}=(-s)+(-q)
$$

как выше, поскольку обе пары $s, p$ и $s, q$ пересекают $\mathcal{O}$. Как $p_{1}$, так и $q_{1}$ пересекает $s$. Итак, три эти линии входят в конфигурацию двойного отражения вместе с $z$. По определению положим

$$
s+x=x+s=z .
$$

В следующей лемме описывается одно из важнейших свойств этой операции. 


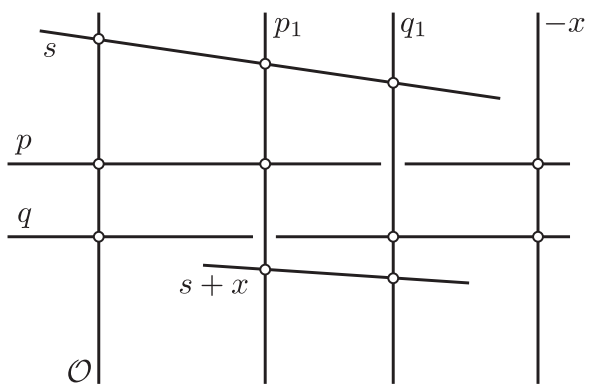

Рис. 23. Частично определенная операция сложения

Лемма 3.21. Пусть $s, x$ - прямые соответственно в $\mathcal{C}_{\mathcal{O}}$ и $\mathcal{A}_{\ell}$, a $\mathcal{Q}_{s}-$ такая квадрика из софокусного семейства, что s и $\mathcal{O}$ получаются друг из друга отражением относительно $\mathcal{Q}_{s}$. Тогда прямая $s+x$ пересекает прямую $-x$, точка их пересечения лежит на квадрике $\mathcal{Q}_{s}$ и две эти прямые удовлетворяют биллиардному закону отражения на $\mathcal{Q}_{s}$.

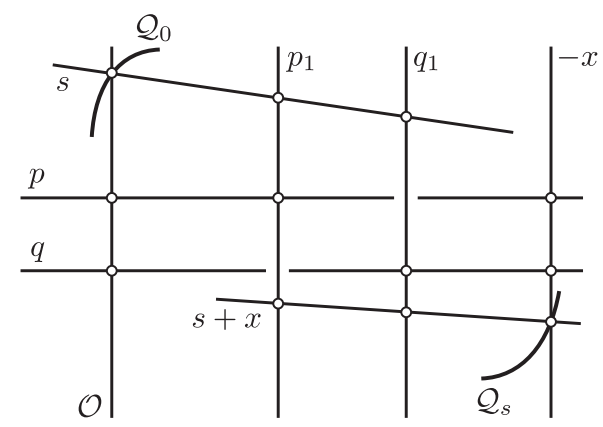

Рис. 24. Лемма 3.21

ДокАЗАТЕЛЬСтво. Это следует из [42], [41] и леммы 3.14.

Лемма 3.21 будет играть важную роль при доказательстве следующих основных свойств так определенной операции сложения.

Лемма 3.22. Для $p, q, s \in \mathcal{C}_{\mathcal{O}}$ выполнен закон ассочиативности: $p+(q+s)=$ $(p+q)+s$.

ДокАЗАТЕЛЬСтво. Как на рис. 24 , обозначим через $x, p_{1}, q_{1}$ прямые, образующие конфигурацию двойного отражения с тройками $(\mathcal{O}, p, q),(\mathcal{O}, p, s)$, $(\mathcal{O}, q, s)$. Применяя лемму 3.21, мы видим, что $(p+q)+s-$ единственная прямая, образующая конфигурацию двойного отражения с тройками $\left(p_{1}, q_{1}, s\right)$, $\left(p, p_{1},-x\right)$ и $\left(q, q_{1},-x\right)$. То же верно для прямой $p+(q+s)$, а значит, эти две прямые совпадают.

Лемма 3.23. Пусть $p, q, s \in \mathcal{C}_{\mathcal{O}}$. Тогда $-(p+q+s)=(-p)+(-q)+(-s)$.

ДокАЗАтельство. Непосредственно проверяется, что $p+q+s \in \mathcal{C}_{\mathcal{O}}$ тогда и только тогда, когда две из прямых $p, q, s$ взаимно обратны. В этом случае нужное нам равенство очевидно. 
Итак, допустим, что $p+q+s$ не пересекает $\mathcal{O}$, а $a$ и $b$ - прямые, образующие конфигурацию двойного отражения с $p+q+s, \mathcal{O}$. По лемме $3.21, p+q+s-$ единственная прямая, пересекающая $(-p)+(-q),(-p)+(-s)$ и $(-q)+(-s)$. Отразив $p, q, s, a, b$ от $\mathcal{Q}_{\mathcal{O}}$ и применив теорему о двойном отражении, мы получаем, что прямая $-(p+q+s)$ пересекает прямые $p+q, p+s$ и $q+s$. Значит, она совпадает с $(-p)+(-q)+(-s)$ по лемме 3.21 .

Пусть теперь даны прямые $x, y \in \mathcal{A}_{\ell}$, не пересекающиеся с $\mathcal{O}$. Их сумма будет определена следующим образом.

Сначала представим $x$ как сумму двух прямых, пересекающих $\mathcal{O}: x=s_{1}+s_{2}$, $s_{1}, s_{2} \in \mathcal{C}_{\mathcal{O}}$. Теперь положим

$$
x+y:=s_{1}+\left(s_{2}+y\right) .
$$

Убедимся в корректности этого определения.

Лемма 3.24. Пусть $s_{1}, s_{2} \in \mathcal{C}_{\mathcal{O}}$, u пусть $y \in \mathcal{A}_{\ell}$. Тогда

$$
s_{1}+\left(s_{2}+y\right)=s_{2}+\left(s_{1}+y\right)
$$

ДокАЗАТЕЛЬСтво. При $y \in \mathcal{C}_{\mathcal{O}}$ достаточно применить лемму 3.22 и свойство коммутативности сложения в $\mathcal{C}_{\mathcal{O}}$.

Если $y \in \mathcal{A}_{\ell} \backslash \mathcal{C}_{\mathcal{O}}$, то прямые $-s_{2}-y$ и $y$ пересекаются и получаются друг из друга биллиардным отражением. То же выполнено и для прямых $-s_{1}-y$ и $y$. Таким образом, существует единственная четвертая прямая, лежащая в пересечении пространства, порожденного $\left[-s_{1}-y, y,-s_{2}-y\right]$, с $\mathcal{A}_{\ell}$. С одной стороны, эта прямая совпадает с $s_{2}-\left(-s_{1}-y\right)$, а с другой, она равна $s_{1}-$ $\left(-s_{2}-y\right)$

Из лемм 3.22-3.24 видно, что мы задали на $\mathcal{A}_{\ell}$ структуру коммутативной группы, естественно связанную с биллиардным законом.

Дальнейшие свойства групповой операции. В начале раздела (с. 174) мы сформулировали основной приниип, к выполнению которого мы стремились при построении алгебры. Теперь мы увидим, что наши усилия увенчались успехом: утверждение, сформулированное как основной принцип, действительно выполняется в построенной группе.

Теорема 3.25. Сумма прямых, входящих в любую конфигурацию двойного отражения, равна нулю.

ДокАЗАТЕЛЬСтво. Пусть $a, b, c, d$ - прямые из конфигурации двойного отражения. Будем считать, что пары $a, b$ и $c, d$ удовлетворяют закону отражения от $\mathcal{Q}_{1}$, а пары $b, c$ и $d, a$ удовлетворяют закону отражения от $\mathcal{Q}_{2}$. Тогда по лемме 3.21 получаем

$$
b=-a+s_{1}, \quad c=-b+s_{2}, \quad d=-c+\bar{s}_{1}, \quad a=-d+\bar{s}_{2},
$$

где $s_{i}, \bar{s}_{i}$ получены из $\mathcal{O}$ биллиардными отражениями от $\mathcal{Q}_{i}, i=1,2$. Очевидно, что $\bar{s}_{i} \in\left\{s_{i},-s_{i}\right\}$. 
Из (3.3) мы получаем

$$
a+b+c+d=s_{1}+\bar{s}_{1}=s_{2}+\bar{s}_{2} .
$$

Таким образом, проверка нужна только в случае, когда $\bar{s}_{1}=s_{1}$ и $\bar{s}_{2}=s_{2}$. Итак, $s_{2}=s_{1}+s_{1}+\left(-s_{2}\right)$ и, как следует из определения операции сложения, прямые $\mathcal{O}, s_{1},-2 s_{1}, s_{2}$ составляют замкнутую биллиардную траекторию с последовательными отражениями от $\mathcal{Q}_{1}, \mathcal{Q}_{1}, \mathcal{Q}_{2}, \mathcal{Q}_{2}$. С другой стороны, $\left(-s_{1}\right)+\left(-s_{2}\right)-$ единственная прямая из $\mathcal{A}_{\ell}$, которая, помимо $\mathcal{O}$, пересекает также $s_{1}$ и $s_{2}$. Таким образом,

$$
-2 s_{1}=\left(-s_{1}\right)+\left(-s_{2}\right) \quad \Rightarrow \quad s_{1}=s_{2} \quad \Rightarrow \quad \mathcal{Q}_{1}=\mathcal{Q}_{2} .
$$

Это означает, что конфигурация двойного отражения $a, b, c, d$ вырождена, т. е. две прямые в ней совпадают. Пусть $a=c$, тогда и $b$ и $d$ получаются из $a$ биллиардным отражением от $\mathcal{Q}_{1}$. Итак, $b=-a+s_{1}, d=-a-s_{1}$ и мы приходим к результату теоремы.

Рассмотрим биллиардную траекторию $\mathbf{t}=\left(\ell_{0}, \ell_{1}, \ldots, \ell_{n}\right)$ с $\ell_{0}=\mathcal{O}$ и с отражениями от квадрик $\mathcal{Q}_{1}, \ldots, \mathcal{Q}_{n}$ из софокусного семейства. Применив теорему о двойном отражении к $\mathbf{t}$, получим различные траектории с тем же начальным отрезком $\mathcal{O}$ и тем же конечным отрезком $\ell_{n}$. Каждая из этих траекторий отражается от того же набора квадрик $\mathcal{Q}_{1}, \ldots, \mathcal{Q}_{n}$, но порядок может быть другим. Обозначим через $\ell_{1}^{(1)}, \ldots, \ell_{n}^{(n)}$ все возможные прямые, содержащие отрезки этих траекторий после первого отражения. Используя нашу алгебру, мы можем вычислить по этим данным конечный отрезок $\ell_{n}$.

ПРЕДЛОЖЕНИЕ 3.26. $\ell_{n}=(-1)^{n+1}\left(\ell_{1}^{(1)}+\cdots+\ell_{n}^{(n)}\right)$.

ДокАЗАТЕльство. Это следует из теоремы 3.25.

Мы также можем вывести из лемм 3.22-3.24 следующее интересное свойство.

ПРЕДЛОЖЕНИЕ 3.27. Пусть $x \in \mathcal{A}_{\ell}-$ прямая, полученная из $\mathcal{O}$ последовательными отражениями от трех квадрик $\mathcal{Q}_{1}, \mathcal{Q}_{2}, \mathcal{Q}_{3}$. Тогда шесть прямых, полученных из $\mathcal{O}$ отражениями от этих квадрик, разбиваются на две группы, такие, что

- при $i \in\{1,2,3\}$ прямые, полученные из $\mathcal{O}$ отражениями от $\mathcal{Q}_{i}$, лежат в разных группах;

- любая траектория, включающая три отражения от $\mathcal{Q}_{1}, \mathcal{Q}_{2}, \mathcal{Q}_{3}$, начинающаяся с $\mathcal{O}$ и кончающаяся на $x$, содержит отрезки прямых только из одной группь.

Обсуждение случая рода 2 мы закончим красивым и нетривиальным результатом о софокусных семействах квадрик.

Теорема 3.28. Пусть $\mathcal{F}$ - семейство софокусных квадрик в $\mathbb{P}^{3}$. Тогда существуют конфигурации 12 плоскостей в $\mathbb{P}^{3}$ со следующими свойствами:

- из этих плоскостей можно составить 8 троек так, чтобы плоскости из одной тройки касались разньх квадрик из $\mathcal{F}$ и три точки касания 
лежали на одной прямой; каждая плоскость конфигуращии войдет в две тройки;

- из этих плоскостей можно составить в 6 четверок так, что плоскости в каждой четверке будут входить в один пучок и касаться двух различных квадрик из $\mathcal{F}$; каждая плоскость конфигурачии войдет в две четверки.

Более того, такая конфигурация задается тремя плоскостями, касающимися трех разных квадрик из $\mathcal{F}$, с точками касания, лежащими на одной прямой.

ДокАЗАтельство. Пусть $\mathcal{O}$ - прямая, содержащая три точки касания, а $p, q, s$ - прямые, полученные из $\mathcal{O}$ биллиардными отражениями от заданных квадрик из $\mathcal{F}$. Построим прямые $p_{1}, q_{1},-x, x+s$ в соответствии с объяснениями перед леммой 3.21 (см. рис. 24). Плоскости из конфигурации касаются соответствующих квадрик в точках пересечения прямых.

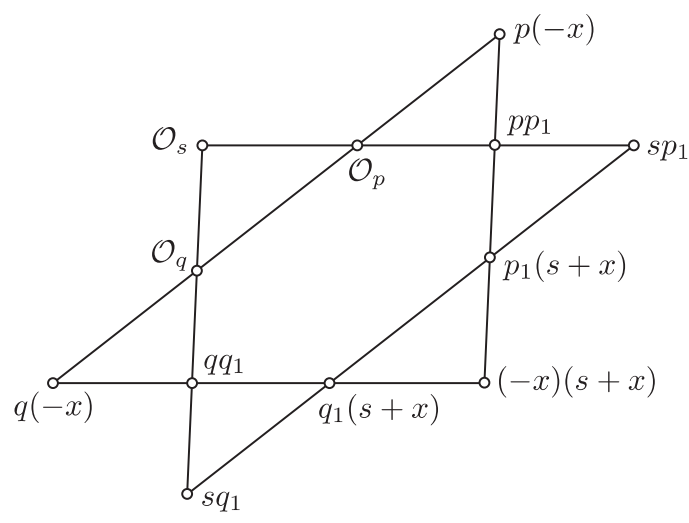

Рис. 25. Конфигурация плоскостей

На рис. 25 изображено представление конфигурации плоскостей в двойственном пространстве $\mathbb{P}^{3 *}$. Здесь каждая плоскость кодируется парой прямых, которые переводятся друг в друга отражением относительно этой плоскости.

Интересно было бы описать многообразие таких конфигураций как пространство модулей.

3.3.3. Общий случай. Разобрав случай рода 2, мы получили необходимый опыт использования биллиардных конструкций для введений структуры группы. Более того, тем самым мы рассмотрели случай $n=2$ общей конструкции, которую мы изложим теперь.

Биллиардные траектории и эффективные дивизоры. При введении групповой операции на множестве $\mathcal{A}_{\ell}$ в более высоких размерностях мы будем мотивированы предложением 3.26. Опишем подробно конструкцию, предшествующую этому предложению.

Пусть $\mathcal{Q}_{1}, \ldots, \mathcal{Q}_{n}$ - квадрики из софокусного семейства в $\mathbb{E}^{d}$. Пусть $\mathcal{O}=\ell_{0}$, $\ell_{1}, \ldots, \ell_{n}-$ такие прямые в $\mathcal{A}_{\ell}$, что каждая пара соседних прямых $\ell_{i}, \ell_{i+1}$ удовлетворяет закону биллиардного отражения от квадрики $\mathcal{Q}_{i+1}(0 \leqslant i \leqslant n-1)$; таким образом, эти прямые задают биллиардную траекторию $\mathbf{t}=\left(\ell_{0}, \ldots, \ell_{n}\right)$.

При $n \geqslant 1$ определим прямые $\ell_{n}^{(n)}, \ell_{n}^{(n-1)}, \ldots, \ell_{n}^{(1)}$ следующим построением. 
1. Положим $\ell_{n}^{(n)}=\ell_{n}$.

2. При $n-1 \geqslant k \geqslant 1$ возьмем в качестве $\ell_{n}^{(k)}$ единственную прямую, образующую конфигурацию двойного отражения с $\ell_{k-1}, \ell_{k}$ и $\ell_{n}^{(k+1)}$.

Заметим, что при этом всякая прямая из последовательности

$$
\ell_{0}, \ell_{1}, \ldots, \ell_{k}, \ell_{n}^{(k+1)}, \ldots, \ell_{n}^{(n)}
$$

получена из предыдущей отражением соответственно от

$$
\mathcal{Q}_{1}, \ldots, \mathcal{Q}_{k}, \mathcal{Q}_{n}, \mathcal{Q}_{k+1}, \ldots, \mathcal{Q}_{n-1}
$$

Рассматривая начальные подпоследовательности $\ell_{0}, \ldots, \ell_{k}(1 \leqslant k \leqslant n)$, мы аналогично определяем прямые $\ell_{k}^{(1)}, \ldots, \ell_{k}^{(k)}$. Заметим, что для всякого $k$ прямая $\ell_{k}^{(1)}$ пересекает $\ell_{0}$ и эти две прямые связаны законом отражения от $\mathcal{Q}_{k}$.

Таким образом, построено отображение $\mathcal{D}$ из множества $\mathcal{T} \mathcal{B}(\mathcal{O})$ биллиардных траекторий с фиксированной начальной прямой $\ell_{0}=\mathcal{O}$ в упорядоченные наборы прямых из $\mathcal{A}_{\ell}$, пересекающих $\mathcal{O}$ :

$$
\mathcal{D}: \quad \mathbf{t}=\left(\ell_{0}, \ldots, \ell_{n}\right) \mapsto\left(\ell_{1}^{(1)}, \ldots, \ell_{n}^{(1)}\right),
$$

где $\ell_{k}^{(1)}$ пересекается с $\mathcal{O}$ в соответствии с биллиардным законом отражения от квадрики $\mathcal{Q}_{k}$.

Проделав обратное построение, мы определим морфизм $\mathcal{B}$, обратный к $\mathcal{D}$, который набору $n$ прямых, пересекающих $\mathcal{O}$, сопоставляет единственную биллиардную траекторию длины $n$ с начальной прямой $\ell_{0}=\mathcal{O}$ :

$$
\mathcal{B}: \quad\left(\ell_{1}^{(1)}, \ldots, \ell_{n}^{(1)}\right) \mapsto\left(\ell_{0}, \ldots, \ell_{n}\right) .
$$

Итак, отображение $\mathcal{B}$ задает биллиардное представление упорядоченного набора прямых, пересекающих $\mathcal{O}$.

Если вместо упорядоченных наборов $n$ прямых мы хотим рассматривать просто дивизоры на кривой $\mathcal{C}_{\mathcal{O}}$, то мы должны ввести следующее отношение $\alpha$ между биллиардными траекториями: будем говорить, что две траектории $\alpha$-эквивалентны, если одна может быть получена из другой конечным набором элементарных перестроек с двойным отражением. Элементарная перестройка с двойным отражением переводит траекторию $p_{1} p_{2} \ldots p_{k-1} p_{k} p_{k+1} \ldots p_{n}$ в траекторию $p_{1} p_{2} \ldots p_{k-1} p_{k}^{\prime} p_{k+1} \ldots p_{n}$, если прямые $p_{k-1}, p_{k}, p_{k}^{\prime}, p_{k+1}$ образуют конфигурацию двойного отражения.

Из теоремы о двойном отражении непосредственно следует, что две траектории $\mathbf{t}_{1}, \mathbf{t}_{2}$ с одним и тем же начальным отрезком $\mathcal{O}=\ell_{0}$ являются $\alpha$-эквивалентными тогда и только тогда, когда $n$-наборы $\mathcal{D}\left(\mathbf{t}_{1}\right)$ и $\mathcal{D}\left(\mathbf{t}_{2}\right)$ получаются один из другого перестановкой.

Итак, $\mathcal{D}$ можно рассматривать как отображение

$$
\widehat{\mathcal{T B}}(\mathcal{O})=\mathcal{T} \mathcal{B}(\mathcal{O}) / \alpha
$$

в множество положительных дивизоров на $\mathcal{C}_{\mathcal{O}}$, и оно задает дивизориалъное представление биллиардных траекторий. 
Следующий результат, вытекающий из [42], является своего рода теоремой Римана-Роха в рамках данного подхода.

Лемма 3.29. Минимальная биллиардная траектория длины $s$ из $x$ в $y$ единственна с точностъю до а-эквивалентности. Если существуют две $\alpha$-неэквивалентных траектории одной длины $k>s$ из $x$ в $y$, то существует траектория из $x$ в у длины $k-2$.

Теперь на множестве $\widehat{\mathcal{T B}}(\mathcal{O})$ мы можем ввести операцию сложения. Для двух биллиардных траекторий $\mathbf{t}_{1}, \mathbf{t}_{2} \in \widehat{\mathcal{T H}}(\mathcal{O})$ мы определим их сумму формулой

$$
\mathbf{t}_{1} \oplus \mathbf{t}_{2}:=\mathcal{B}\left(\mathcal{D}\left(\mathbf{t}_{1}\right)+\mathcal{D}\left(\mathbf{t}_{2}\right)\right)
$$

По теореме о двойном отражении эта операция ассоциативна и коммутативна.

Tеорема 3.30. Множество $\widehat{\mathcal{T B}}(\mathcal{O})=\mathcal{T} \mathcal{B}(\mathcal{O}) / \alpha$ с операцией сложения является коммутативной полугруппой. Она изоморфна полугруппе эффективных дивизоров на кривой $\mathcal{C}_{\mathcal{O}}$, не содержащих точек, соответствующих каустикам.

Заметим, что траектория, состоящая из одной прямой $\mathcal{O}$, будет нулевым элементом в $\widehat{\mathcal{T} \mathcal{B}}(\mathcal{O})$.

Теперь определим следующее отношение эквивалентности на множестве конечных биллиардных траекторий.

ОПРЕДЕЛЕНИЕ 3.31. Две биллиардные траектории $\beta$-эквивалентны, если их начальные и конечные отрезки совпадают и эти траектории имеют одинаковую длину.

Мы обозначим множество классов $\beta$-эквивалентных биллиардных траекторий длины $n$ с фиксированным начальным отрезком $\mathcal{O}$ через $\widetilde{\mathcal{T}} \mathcal{B}(\mathcal{O})(n)$ и положим

$$
\widetilde{\mathcal{T} \mathcal{B}}(\mathcal{O})=\bigcup_{n} \widetilde{\mathcal{T} \mathcal{B}}(\mathcal{O})(n)
$$

ПРЕДЛОЖЕНИЕ 3.32. Отношение $\beta$ согласуется со сложением биллиардных траекторий.

Для доказательства нам потребуется следующая важная лемма.

Лемма 3.33. Пусть $\mathbf{t}=\left(\ell_{1}, \ell_{2}, \ldots, \ell_{2 k}, \ell_{2 k+1}=\ell_{1}\right)$ - замкнутая биллиардная траектория, а $p_{1} \in \mathcal{A}_{\ell}-$ прямая, пересекающая $\ell_{1}$. Итеративной процедурой построим прямые $p_{2}, \ldots, p_{2 k+1}$, для которых четверки $p_{i}, \ell_{i}, p_{i+1}, \ell_{i+1}$ $(1 \leqslant i \leqslant 2 k)$ образуют конфигурачию двойного отражения. Тогда $p_{2 k+1}=p_{1}$.

ДокАЗАТЕЛЬСтво. Будем рассуждать по индукции. При $k=2$ прямые $\ell_{1}$, $\ell_{2}, \ell_{3}, \ell_{4}$ образуют конфигурацию двойного отражения и результат следует по теореме о двойном отражении. 
Пусть теперь $k>2$. Тогда $\left(\ell_{1}, \ldots, \ell_{k}\right)$ и $\left(\ell_{1}=\ell_{2 k+1}, \ell_{2 k}, \ldots, \ell_{k}\right)$ - это биллиардные траектории одной длины из $\ell_{1}$ в $\ell_{k}$. Если они $\alpha$-эквивалентны, то утверждение следует из теоремы о двойном отражении. Если они $\alpha$-неэквивалентны, то по лемме 3.29 мы имеем биллиардную траекторию $\ell_{1}^{\prime}=\ell_{1}, \ell_{2}^{\prime}, \ldots, \ell_{k-2}^{\prime}=\ell_{k}$. Теперь утверждение леммы следует по предположению индукции для траекторий

$$
\left(\ell_{1}, \ell_{2}, \ldots, \ell_{k}=\ell_{k-2}^{\prime}, \ell_{k-3}^{\prime}, \ldots, \ell_{1}^{\prime}=\ell_{1}\right)
$$

и

$$
\left(\ell_{1}^{\prime}, \ell_{2}^{\prime}, \ldots, \ell_{k-2}^{\prime}=\ell_{k}, \ell_{k+1}, \ldots, \ell_{2 k+1}=\ell_{1}\right) .
$$

ДокАЗАТЕЛЬСТво предложения 3.32. Мы должны доказать импликацию

$$
\mathbf{t}_{1} \sim_{\beta} \mathbf{t}_{1}^{\prime}, \mathbf{t}_{2} \sim_{\beta} \mathbf{t}_{2}^{\prime} \Rightarrow \mathbf{t}_{1}+\mathbf{t}_{2} \sim_{\beta} \mathbf{t}_{1}^{\prime}+\mathbf{t}_{2}^{\prime} .
$$

Ясно, что достаточно доказать ее в случае, когда $\mathbf{t}_{2}=\mathbf{t}_{2}^{\prime}$, a $\mathbf{t}_{2}$ имеет длину 2 . Пусть $\mathbf{t}_{2}=(\mathcal{O}, p)$, где $p$ получена из $\mathcal{O}$ отражением от квадрики $\mathcal{Q}_{p}$. Тогда траектории $\mathbf{t}_{1}+\mathbf{t}_{2}$ и $\mathbf{t}_{1}+\mathbf{t}_{2}^{\prime}$ получены добавлением к $\mathbf{t}_{1}$ и $\mathbf{t}_{1}^{\prime}$ по одному отрезку. Эти отрезки связаны с последними отрезками траекторий $\mathbf{t}_{1}, \mathbf{t}_{1}^{\prime}$ законом отражения от $\mathcal{Q}_{p}$. Поскольку $\mathbf{t}_{1} \sim_{\beta} \mathbf{t}_{1}^{\prime}$, их последние отрезки совпадают и предложение следует из леммы 3.33 для траектории

$$
\left(\ell_{1}, \ell_{2}, \ldots, \ell_{n}=\ell_{n}^{\prime}, \ell_{n-1}^{\prime}, \ell_{n-2}^{\prime}, \ldots, \ell_{1}^{\prime}=\ell_{1}\right)
$$

где $\mathbf{t}_{1}=\left(\ell_{1}, \ldots, \ell_{n}\right), \mathbf{t}_{2}=\left(\ell_{1}^{\prime}, \ldots, \ell_{n}^{\prime}\right)$.

Структура группы на $\mathcal{A}_{\ell}$. Мы хотим использовать алгебру, построенную на множестве биллиардных траекторий, чтобы ввести алгебраическую структуру на $\mathcal{A}_{\ell}$ с операцией, естественно связанной с законом биллиардного отражения.

Следующий результат содержится в [73].

Теорема 3.34. Для произвольных прямых $x$ и у из $\mathcal{A}_{\ell}$ существует такой набор из не более чем $d-1$ квадрик из софокусного семейства, что у получается из $x$ последовательными отражениями от этих квадрик.

Дивизориальное представление соответствующей биллиардной траектории длины $s \leqslant d-1$ назовем $s$-щеткой $y$ относительно $x$.

Теперь мы можем задать на $\mathcal{A}_{\ell}$ структуру группы, привязанную к фиксированной прямой из этого множества.

Нейтралъный элемент. Фиксируем прямую $\mathcal{O} \in \mathcal{A}_{\ell}$.

Обратный элемент. Пусть $x$ - произвольная прямая в $\mathcal{A}_{\ell}$, а $\mathcal{D}(x)$ - дивизориальное представление минимальной биллиардной траектории, связывающей $\mathcal{O}$ с $x$. Определим $-x$ как последний отрезок биллиардной траектории $\mathcal{B}(\tau \mathcal{D}(x))$, где $\tau$ - гиперэллиптическая инволюция $\mathcal{C}_{\mathcal{O}}$.

Сложение. Для двух прямых $x$ и $y$ из $\mathcal{A}_{\ell}$ обозначим их щетки относительно $\mathcal{O}$ через $S_{1}$ и $S_{2}$. Положим

$$
x+y:=(-1)^{\left|S_{1}\right|+\left|S_{2}\right|+1} \mathcal{E} \mathcal{B}\left(\tau^{\left|S_{1}\right|+1}\left(S_{1}\right), \tau^{\left|S_{2}\right|+1}\left(S_{2}\right)\right),
$$

где $\mathcal{E} \mathcal{B}\left(\tau^{\left|S_{1}\right|+1}\left(S_{1}\right), \tau^{\left|S_{2}\right|+1}\left(S_{2}\right)\right)$ - последний отрезок биллиардной траектории $\mathcal{B}\left(\tau^{\left|S_{1}\right|+1}\left(S_{1}\right), \tau^{\left|S_{2}\right|+1}\left(S_{2}\right)\right)$. 
Из сказанного выше, аналогично случаю рода 2 , получаем следующее утверждение.

Теорема 3.35. Множество $\mathcal{A}_{\ell}$ с определенной выше операцией является абелевой әруппой.

Заметим, что можно ввести еще одно дивизориальное представление биллиардных траекторий, сопоставляющее траектории дивизор степени 0. Это представление использовалось в [44] и было явно описано в [26], [29]. Положительная компонента дивизора в этом представлении совпадает с дивизором, полученным с помощью $\mathcal{D}$, а отрицательная часть инвариантна относительно гиперэллиптической инволюции. Более того, как следует из [44], две траектории с начальным отрезком $\mathcal{O}$ имеют один и тот же конечный отрезок тогда и только тогда, когда их представления являются эквивалентными дивизорами. Отсюда следует, что групповая структура на $\mathcal{A}_{\ell}$ изоморфна фактору якобиана кривой $\mathcal{C}_{\mathcal{O}}$ по конечной подгруппе, порожденной точками, соответствующими квадрикам-каустикам.

3.4. s-слабые траектории Понселе. Как следует из теоремы 3.34, мы можем теперь ввести следующие иерархии понятий.

ОПреДЕЛЕНИЕ 3.36. Для данных прямых $x$ и $y$ из $\mathcal{A}_{\ell}$ скажем, что они $s$-скрещены, если $s$ - наименьшее число, для которого существует такой набор из $s+1 \leqslant d-1$ квадрик $\mathcal{Q}_{k}, k=1, \ldots, s+1$, из софокусного семейства, что прямая $y$ получена из $x$ последовательными отражениями от $\mathcal{Q}_{k}$. Если прямые $x$ и $y$ пересекаются, то они 0-скрещены. Они $(-1)$-скрещены, если совпадают.

ОПРЕДЕЛЕНИЕ 3.37. Пусть $S$ - система из $n$ квадрик $\mathcal{Q}_{1}, \ldots, \mathcal{Q}_{n}$ софокусного семейства. Для системы прямых $\mathcal{O}_{0}, \mathcal{O}_{1}, \ldots, \mathcal{O}_{n}$ в $\mathcal{A}_{\ell}$, где каждая пара соседних прямых $\mathcal{O}_{i}, \mathcal{O}_{i+1}$ удовлетворяет биллиардному закону отражения от $\mathcal{Q}_{i+1}$ $(0 \leqslant i \leqslant n-1)$, мы скажем, что она образует $s$-слабую траекторию Понселе длины $n$, ассочиированную с системой $S$, если прямые $\mathcal{O}_{0}$ и $\mathcal{O}_{n} s$-скрещены.

$s$-слабые траектории Понселе мы будем иногда называть $(d-s-2)$-резонансными биллиардными траекториями. Периодические траектории, т. е. обобщенные классические многоугольники Понселе, являются (-1)-слабыми траекториями Понселе или, другими словами, $(d-1)$-резонансными биллиардными траекториями; они аналитически описаны в [29], [26].

Очередной нашей задачей является аналитическое описание $s$-слабых траекторий Понселе длины $r$, что станет обобщением результатов из [29]. Здесь мы в полной мере используем методы и возможности биллиардной алгебры.

Чтобы дать представление об основной идее, рассмотрим сначала систему $S$ из $r$ одинаковых квадрик $\mathcal{Q}_{1}=\cdots=\mathcal{Q}_{r}$ софокусного семейства. Пусть набор прямых $\mathcal{O}_{0}, \mathcal{O}_{1}, \ldots, \mathcal{O}_{r}$ из $\mathcal{A}_{\ell}$ образует $s$-слабую траекторию Понселе длины $r$, ассоциированную с набором $S$. Тогда

$$
\mathcal{O}_{r}=r \mathcal{O}_{1}^{(1)}
$$

для некоторой прямой $\mathcal{O}_{1}^{(1)}$, пересекающей $\mathcal{O}_{0}$. Снова пользуясь условием $s$-скрещенности $\mathcal{O}_{r}$ и $\mathcal{O}_{0}$, получаем

$$
\mathcal{O}_{r}=\mathcal{O}_{1}^{\prime(1)}+\cdots+\mathcal{O}_{s+1}^{\prime(1)}
$$


где $\mathcal{O}_{i}^{(1)}=: P_{i}$ - некоторые прямые, пересекающие $\mathcal{O}_{0}$. Два последних уравнения приводят нас к следующему выводу.

ПреДЛОЖЕНИЕ 3.38. Существование s-слабой траектории Понселе длины $r$ эквивалентно существованию такой мероморфной функции $f$ на гиперэллиптической кривой $\mathcal{C}_{\ell}$, что $f$ имеет нуль порядка в в $P=\mathcal{O}_{1}^{(1)}$ и единственный полюс на "бесконечности" $E$, порядок полюса равен $n=r+s+1$.

Теперь мы выведем явное аналитическое условие типа Кэли. Как и в [26], рассмотрим пространство $\mathcal{L}(n E)$ мероморфных функций на $\mathcal{C}_{\ell}$, имеющих единственный полюс порядка не выше $n$ в бесконечно удаленной точке $E$. Пусть $\left(f_{1}, \ldots, f_{k}\right)$ - некоторый базис в этом пространстве, $k=\operatorname{dim} \mathcal{L}(n E)$. Рассмотрим векторы

$$
v_{1}, \ldots, v_{r} \in \mathbb{C}^{k}
$$

где $v_{i}^{j}=f_{j}^{(i-1)}(P)$, и векторы

$$
u_{1}, \ldots, u_{s+1} \in \mathbb{C}^{k}
$$

где $u_{i}^{j}=f_{j}\left(P_{i}\right)$. Из условия (см. [26])

$$
\operatorname{rank}\left[v_{1}, \ldots, v_{r}, u_{1}, \ldots, u_{s+1}\right]<n-g+1
$$

получаем

$$
\operatorname{rank}\left[v_{1}, \ldots, v_{r}\right]<r+s-g+2=r+s-d+3
$$

Мы можем переписать это условие в форме привычной для условий типа Кэли.

Теорема 3.39. Существование s-слабой траектории Понселе длины $r$ эквивалентно неравенству

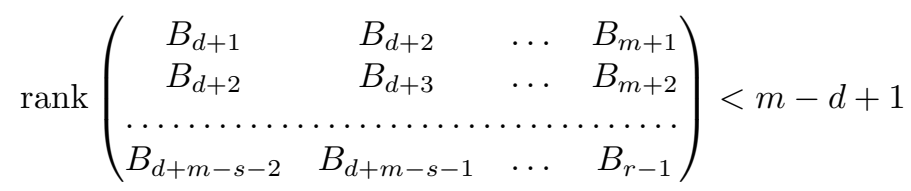

при $r+s+1=2 m$ и неравенству

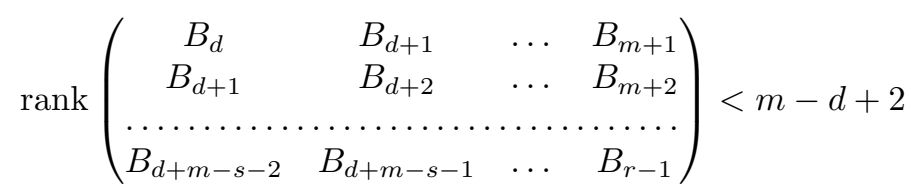

npu $r+s+1=2 m+1$.

Через $B_{0}, B_{1}, B_{2}, \ldots м ы$ обозначаем здесь коэффициенты тейлоровского разложения функции $y=\sqrt{\mathcal{P}(x)}$ в окрестности $P$, где $y^{2}=\mathcal{P}(x)$ - уравнение обобщенной кривой Кэли, а $\mathcal{P}(x)$ - полином, заданный в (2.2). 
ДоказАтельство. Пусть $\mathcal{L}((r+s+1) E)$ - линейное пространство мероморфных функций на $\mathcal{C}_{\ell}$ с единственным полюсом в бесконечно удаленной точке $E$, порядка не выше $r+s+1$. По теореме Римана-Роха

(i) $\operatorname{dim} \mathcal{L}((r+s+1) E)=\left[\frac{r+s+1}{2}\right]+1$ при $r+s+1 \leqslant 2 d-1$,

(ii) $\operatorname{dim} \mathcal{L}((r+s+1) E)=r+s-g+1$ при четном $r+s+1$, превосходящем $2 d-2$,

(iii) $\operatorname{dim} \mathcal{L}((r+s+1) E)=r+s-g+2$ при нечетном $r+s+1$, превосходящем $2 d-1$.

В этих трех случаях мы можем выбрать соответственно следующие базисы:

(i) $1, x, \ldots, x^{m}$, где $m=\left[\frac{r+s+1}{2}\right] \leqslant d$;

(ii) $1, x, \ldots, x^{m}, y, x y, \ldots, x^{m-d}$, при $r+s+1=2 m \geqslant 2 d-2$;

(iii) $1, x, \ldots, x^{m}, y, x y, \ldots, x^{m-d+1}$ при $r+s+1=2 m+1 \geqslant 2 d-2$.

Теперь утверждение теоремы следует из рассуждений, предшествующих формулировке.

ПримеР 3.40. При $s=-1$ неравенства в теореме 3.39 оказываются условиями периодичности биллиардной траектории (см. [26], [29]).

ПримеР 3.41. Условие существования $(d-3)$-слабой траектории Понселе длины $r$ эквивалентно равенствам

$$
\begin{aligned}
& \operatorname{det}\left(\begin{array}{cccc}
B_{d+1} & B_{d+2} & \ldots & B_{m+1} \\
B_{d+2} & B_{d+3} & \ldots & B_{m+2} \\
\ldots \ldots & \ldots \ldots \ldots & \ldots & \ldots \ldots \\
B_{m+1} & B_{m+2} & \ldots & B_{r-1}
\end{array}\right)=0, \quad r+d-2=2 m ; \\
& \operatorname{det}\left(\begin{array}{cccc}
B_{d} & B_{d+1} & \ldots & B_{m+1} \\
B_{d+1} & B_{d+2} & \ldots & B_{m+2} \\
\ldots \ldots & \ldots \ldots & \ldots & \ldots \ldots \\
B_{m+1} & B_{m+2} & \ldots & B_{r-1}
\end{array}\right)=0, \quad r+d-2=2 m+1 .
\end{aligned}
$$

ПримеР 3.42. При $r+s<2 d-1$, как следует из доказательства теоремы $3.39, s$-слабая траектория Понселе длины $r$ может существовать только когда гиперэллиптическая кривая $\mathcal{C}_{\ell}$ особая.

\section{5. Обобщенная теорема Вейра и пространственная теорема Пон-} селе в форме Гриффитса-Харриса в больших размерностях. В этом пункте мы получим многомерные обобщения результатов из [43], [75], [4]. Хороший обзор по этим классическим результатам можно найти в [18]. Двойственная версия [4] приведена в [21].

Каждая квадрика $\mathcal{Q}$ из $\mathbb{P}^{2 d-1}$ содержит не более двух унирациональных семейств $(d-1)$-мерных линейных подпространств. Такие унирациональные семейства обычно называют прямолинейными образующими квадрики.

Теорема 3.43. Пусть $\mathcal{Q}_{1} u \mathcal{Q}_{2}$ - две общие квадрики в $\mathbb{P}^{2 d-1}$ с гладким пересечением $V$, и пусть $\mathcal{R}_{1}, \mathcal{R}_{2}$ - их прямолинейные образуюшие. Если существует такая замкнутая чепочка

$$
L_{1}, L_{2}, \ldots, L_{2 n}, L_{2 n+1}=L_{1}
$$


различных $(d-1)$-мерных линейных подпространств, что $L_{2 i-1} \in \mathcal{R}_{1}, L_{2 i} \in \mathcal{R}_{2}$ $(1 \leqslant i \leqslant n)$ и $L_{j} \cap L_{j+1} \in F(V)(1 \leqslant j \leqslant 2 n)$, то такие замкнутые цепочки подпространств длины $2 n$ проходят через каждую точку $F(V)$.

ДокАЗАтЕльство. Каждое из унирациональных семейств $\mathcal{R}_{i}$ задает инволюцию $\tau_{i}$ на абелевом многообразии $F(V)$. Эта инволюция переставляет два $(d-2)$-мерных подпространства в пересечении образующей $\mathcal{R}_{i}$ с $V$. Пусть $\operatorname{Tr}: F(V) \rightarrow F(V)$ - композиция этих инволюций, а $L:=L_{2 n} \cap L_{1} \in F(V)$. Поскольку $\operatorname{Tr}$ - сдвиг на $F(V)$, для которого $\operatorname{Tr}^{n}(L)=L$, мы видим, что $\operatorname{Tr}$ имеет порядок $n$, что и дает утверждение теоремы.

ОПРЕДЕЛЕНИЕ 3.44. Цепочки, рассмотренные в теореме 3.43, назовем обобщенными цепочками Вейра.

Эту теорему можно сформулировать и для негладких пересечений, но мы не будем в это вдаваться. Вместо этого рассмотрим случай двух квадрик (3.1) и (3.2), как в п. 3.3. Используя проекцию $\pi^{*}$, получаем следующий результат.

ПРЕДЛОЖЕНИЕ 3.45. Обобщенная цепочка Вейра длины $2 n$ проецируется на многоугольник Понселе длины $2 n$, описанный около квадрик $\mathcal{Q}_{\alpha_{1}}^{p}, \ldots, \mathcal{Q}_{\alpha_{d-1}}^{p}$ и попеременно вписанный в две фиксированные софокусные квадрики (проекции $\left.\mathcal{Q}_{1}, \mathcal{Q}_{2}\right)$. Наоборот, всякий такой многоугольник Понселе длины $2 n$, описанный около квадрик $\mathcal{Q}_{\alpha_{1}}^{p}, \ldots, \mathcal{Q}_{\alpha_{d-1}}^{p}$ и попеременно вписанный в две фиксированные софокусные квадрики, можно поднять до обобщенной чепочки Вейра длины $2 n$.

ДокАзАТЕльство. Утверждение вытекает из [41; лемма 4.1, следствие 4.2] и леммы 3.14 .

Таким образом, мы получаем соответствие между обобщенными цепочками Вейра и многоугольниками Понселе, описанными около $d-1$ фиксированной квадрики и попеременно вписанными в две квадрики из софокусного семейства. Такие многоугольники Понселе были в числе прочего полностью аналитически описаны в [29] (см. пример 4 в этой работе).

Заметим, что соответствие между классической теоремой Вейра в $\mathbb{P}^{3}$ и классической теоремой Понселе о двух кониках на плоскости было отмечено Гурвицем в [75]. Однако используемая нами здесь проекция не является непосредственным обобщением проекции, использованной Гурвицем.

Ломаные, описанные около коники и попеременно вписанные в две другие коники, возникают в [31] в качестве примера. Наш результат в минимальной размерности дает условие замкнутости такой ломаной; см. следствие 1 в [29].

Укажем также, что если прямолинейные образующие $\mathcal{R}_{1}$ и $\mathcal{R}_{2}$ связаны обобщенной цепочкой Вейра длины $2 n$, то то же выполнено для $\mathcal{R}_{2}, \mathcal{R}_{1}$ и для пары $\mathcal{R}_{1}^{\prime}, \mathcal{R}_{2}^{\prime}$ "дополнительных" семейств прямолинейных образующих на $\mathcal{Q}_{1}$ и $\mathcal{Q}_{2}$.

Теперь мы можем сформулировать новое многомерное обобщение пространственной теоремы Понселе, принадлежащей Гриффитсу и Харрису [4].

ТеОрема 3.46. Пусть $\mathcal{Q}_{1}^{*}$ и $\mathcal{Q}_{2}^{*}$ двойственны $\kappa$ общим квадрикам в $\mathbb{P}^{2 d-1}$ с гладким пересечением $V$. Обозначим через $\mathcal{R}_{i}, \mathcal{R}_{i}^{\prime}$ пары унирациональньх семейств $(d-1)$-мерных подпространств, лежащих в $\mathcal{Q}_{i}^{*}$. Предположим, что $\mathcal{R}_{1}$ и $\mathcal{R}_{2}$, а такэе $\mathcal{R}_{1}$ и $\mathcal{R}_{2}^{\prime}$ связаны обобщенными цепочками Вейра. Тогда существует конечная конфигурация, вписанная и описанная в обе квадрики $\mathcal{Q}_{1}$ u $\mathcal{Q}_{2}$. Таких конфигураций бесконечно много. 
"Конфигурации" из предыдущей теоремы могут быть описаны детальнее как конфигурации $d$-мерных "граней", бикасательных к квадрикам $\mathcal{Q}_{1}$ и $\mathcal{Q}_{2}$. Их пересечения - $(d-1)$-мерные "ребра", попеременно принадлежащие семействам прямолинейных образующих на $\mathcal{Q}_{1}^{*}$ и $\mathcal{Q}_{2}^{*}$. Пересечения "ребер” - это $(d-2)$-мерные “вершины”, принадлежащие $F(V)$.

Доказательство последней теоремы основано на следующей лемме из [42].

Лемма 3.47. Пусть $\mathcal{Q} \subset \mathbb{P}^{2 d-1}$ - квадрика ранга не менъше $2 d-1 u x \subset \mathcal{Q}$ линейное подпространство размерности $d-2$. Если $\mathcal{Q}$ особая, то дополнительно допустим, что $x$ не содержит вершины. Тогда для всякой системы образующих $\mathcal{R}$ на $\mathcal{Q}$ существует единственное $(d-1)$-мерное линейное подпространство $s=s(\mathcal{R}, x)$ с тем свойством, что $s \in \mathcal{R}$ u $x \subset s$.

\section{6. Сетка Понселе-Дарбу и многомерные обобщения.}

3.6.1. Историческое замечание о наследии Дарбу. В [44] (том 3, книга VI, гл. I) Дарбу показал, что поверхности Лиувилля - это в точности класс поверхностей, на которых имеется ортогональная система кривых, которые можно рассматривать как геодезические коники двумя или, что эквивалентно, бесконечным числом способов. Эти координатные кривые являются аналогами систем софокусных коник в евклидовой плоскости. Ввиду этого, важнейшие свойства коник можно обобщить на все поверхности Лиувилля (см. [44], а также обзор и разъяснения в [29]). Вот цитата с описанием одного из таких свойств:

"Рассмотрим переменный многоугольник, все стороны которого являются геодезическими, касающимися одной и той же координатной кривой. Тогда последняя вершина многоугольника будет описывать некоторую координатную кривую и тем же свойством будут обладать точки пересечения произвольных пар сторон многоугольника".

Здесь мы видим не только обобщение теоремы Понселе для поверхностей Лиувилля. Сверх того для многоугольника Понселе, описанного около фиксированной координатной кривой, каждая вершина которого движется по какой-либо координатной кривой, Дарбу утверждает, что точка пересечения произвольной пары его сторон также будет описывать координатную кривую.

Заметим, что ослабленная форма этого утверждения, хотя и с некоторыми усовершенствованиями, недавно была переоткрыта в [76], а более элементарное доказательство результата из [76] было опубликовано в [77]. Основная теорема работы [76] соответствует случаю фиксированного многоугольника Понселе, связанного с парой эллипсов в евклидовой плоскости. В каком-то смысле это еще одно свидетельство в пользу нашего замечания в [26] и [29], что работы Дарбу по биллиардам и теореме Понселе, по-видимому, неизвестны современным математикам.

Этот пункт посвящен многомерному обобщению теоремы Дарбу, связанному с биллиардными траекториями внутри эллипсоида в $d$-мерном евклидовом пространстве.

3.6.2. Сетка Понселе-Дарбу в евклидовой плоскости. Перед тем как перейти к многомерным обобщениям, изложим здесь чуть более элементарное доказательство некоторого усиления соответствующего результата в плоском случае. 

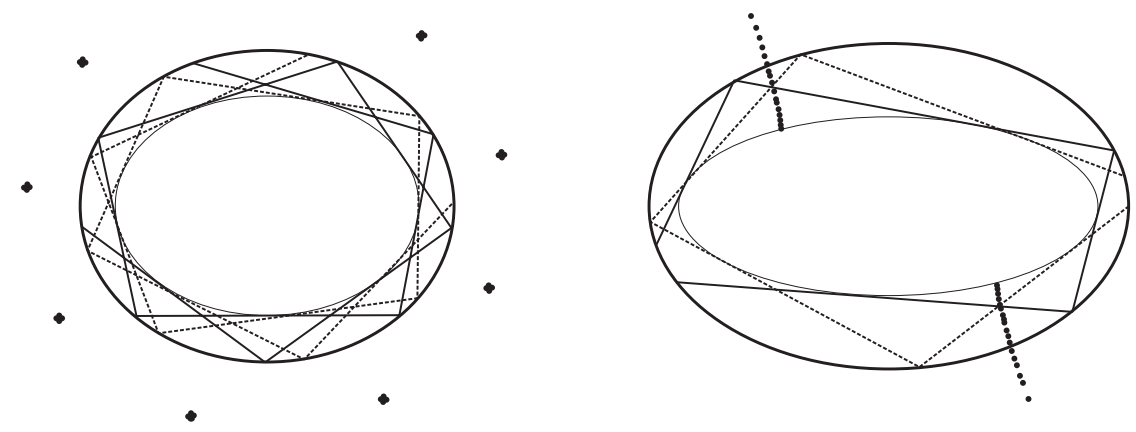

Рис. 26. Биллиардные траектории с каустикой-эллипсом и точки пересечения соответствующих отрезков
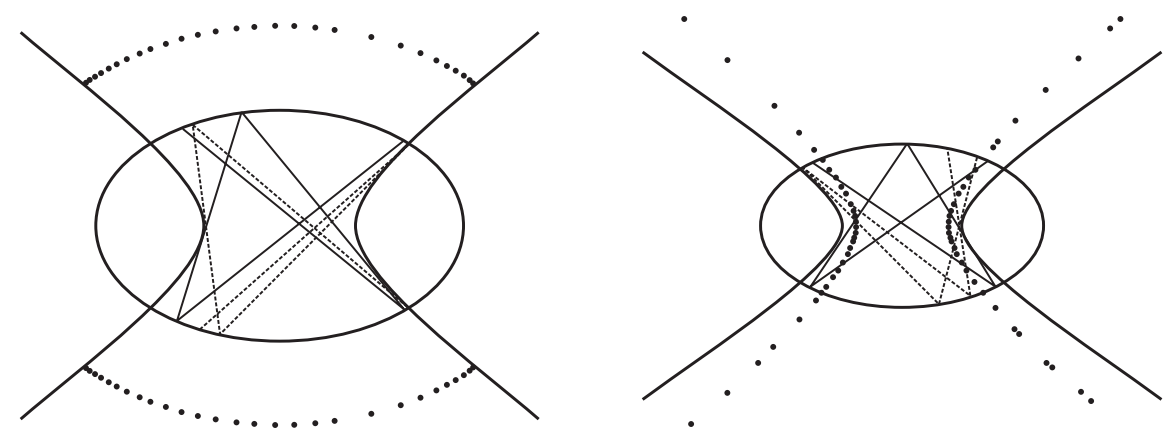

Рис. 27. Биллиардные траектории с каустикой-эллипсом и точки пересечения соответствующих отрезков

Теорема 3.48. Пусть $\mathcal{E}$ - эллипс в $\mathbb{E}^{2}$, a $\left(a_{m}\right)_{m \in \mathbb{Z}},\left(b_{m}\right)_{m \in \mathbb{Z}}-$ последовательности отрезков биллиардных траекторий в $\mathcal{E}$ с одной и той же каустикой. Тогда все точки $a_{m} \cap b_{m}(m \in \mathbb{Z})$ лежат на одном и том же коническом сечении $\mathcal{K}$, софокусном с $\mathcal{E}$.

Более того, если дополнительно предположить, что каустика - эллипс, и если обе траектории обходят каустику в одном и том же направлении, то $\mathcal{K}$ - также эллипс; если же траектории обходят каустику в противоположных направлениях, то $\mathcal{K}$ - гипербола (см. рис. 26).

Если же каустика является гиперболой, а отрезки $a_{m}, b_{m}$ пересекают большую осъ $\mathcal{E}$ в одном и том же направлении, то $\mathcal{K}$ - гипербола, в противном случае это эллипс (см. рис. 27).

ДокАЗАТЕЛЬСтво. Этот результат доказывается применением теоремы о двойном отражении. А именно, прямые $a_{0}$ и $b_{0}$ пересекаются в точке, лежащей на некотором эллипсе и некоторой гиперболе из софокусного семейства. Они удовлетворяют закону отражения от какой-то одной из этих кривых, в зависимости от направления биллиардного движения вдоль прямых. Теперь по теореме о двойном отражении $a_{1}$ и $b_{1}$ удовлетворяют закону отражения от той же коники. То же выполнено по индукции и для всякой пары $a_{m}, b_{m}$. 
Для доказательства второй части теоремы достаточно заметить, что направление обхода эллипса меняется при отражении от гипербол и сохраняется при отражении от эллипсов из софокусного семейства. Если ориентированная прямая проходит между фокусами, то направление, в котором она пересекает ось, содержащую фокусы, меняется при отражении от эллипсов и сохраняется при отражении от гипербол.

ПРЕДЛОЖЕНИЕ 3.49. Пусть $\left(a_{m}\right)_{m \in \mathbb{Z}},\left(b_{m}\right)_{m \in \mathbb{Z}}-$ две последовательности отрезков биллиардных траекторий внутри эллипса $\mathcal{E}$, имеющих одну и ту же каустику. Если эта каустика - эллипс, а траектории обходят его в противоположных направлениях, то все точки $a_{m} \cap b_{m}(m \in \mathbb{Z})$ лежат на двух симметричных относительно центра четвертях гиперболы, софокусной с $\mathcal{E}$.

ДокАЗАТЕльство. Обозначим через $\mathcal{E}_{c}$ эллипс, являющийся общей каустикой рассматриваемых биллиардных траекторий. Мы можем ввести метрику $\mu$ на $\mathcal{E}_{c}$, в которой $\mu(A B)=\mu(C D)$ тогда и только тогда, когда касательные в $A, B$ и $C, D$ пересекаются в точках одного и того же эллипса из софокусного семейства. В частности, это значит, что точки, в которых два последовательных отрезка траектории касаются каустики $\mathcal{E}^{\prime}$, всегда расположены на одном и том же расстоянии друг от друга.

Допустим, что $a_{0}$ и $b_{0}$ пересекаются в точке верхней левой четверти гиперболы $\mathcal{K}$ (см. рис. 26). Тогда, используя теорему о двойном отражении, можно доказать, что всякая пара точек касания таких отрезков с каустикой $\mathcal{E}^{\prime}$ лежит на равном $\mu$-расстоянии от верхней левой точки пересечения $\mathcal{K}$ и $\mathcal{E}_{c}$. Поскольку $\mu$ симметрична относительно начала координат, они также равноудалены от нижней правой точки пересечения, но не равноудалены от двух других точек пересечения. То же выполняется для любой пары $a_{m}, b_{m}$. Итак, их точки пересечения лежат на двух центрально симметричных четвертях гиперболы.

Теперь обобщенное утверждение о сетках Понселе-Дарбу оказывается непосредственным следствием теоремы 3.48 .

ТЕОРема 3.50. Пусть $\left(\ell_{m}\right)_{m \in \mathbb{Z}}$ - последовательность отрезков биллиардной траектории внутри эллипса $\mathcal{E}$. Тогда каждое из множеств

$$
\mathrm{P}_{k}=\bigcup_{i-j=k} \ell_{i} \cap \ell_{j}, \quad \mathrm{Q}_{k}=\bigcup_{i+j=k} \ell_{i} \cap \ell_{j} \quad(k \in \mathbb{Z})
$$

лежит на некоторой конике, софокусной с $\mathcal{E}$.

Если каустика траектории $\left(\ell_{m}\right)$ - эллипс, то множества $\mathrm{P}_{k}$ лежат на эллипсах, а $\mathrm{Q}_{k}$ лежсат на гиперболах. Если эта каустика - гипербола, то множества $\mathrm{P}_{k}, \mathrm{Q}_{k}$ лежат на эллипсах при четных $k$ и на гиперболах при нечетHoux $k$.

ДокАзАТЕльство. Для доказательства утверждения относительно $\mathrm{P}_{k}$ положим $a_{m}=\ell_{m}, b_{m}=\ell_{m+k}$ и применим теорему 3.48. Для $\mathrm{Q}_{k}$ положим $a_{m}=\ell_{m}$ и $b_{m}=\ell_{k-m}$.

ЗАмЕчАниЕ 3.51. Заметим, что в теореме 3.50 доказан более общий результат, чем в [76], [77], поскольку мы не требуем замкнутости биллиардной траектории. Кроме того, его можно сформулировать для любого конического сечения, не обязательно эллипса. 
Основной результат [76] и [77] - это частное следствие теоремы 3.50:

СлеДСТВИЕ 3.52 [76], [77]. Пусть $\left(\ell_{m}\right)$ - замкнутая биллиардная траектория внутри эллипса с каустикой-эллипсом. Тогда каждое множество $\mathrm{P}_{k}$ лежит на эллипсе, софокусном $c \mathcal{E}$, a $\mathrm{Q}_{k}$ лежит на софокусной гиперболе. (C.м. puc. 26.)

Продемонстрируем интересное свойство сеток Понселе-Дарбу.

ПрЕДЛОЖЕНИЕ 3.53. Пусть $\left(\ell_{m}\right)$ - биллиардная траектория в эллипсе $\mathcal{E}$ с каустикой-эллипсом $\mathcal{E}_{c}$. Тогда эллипс, содержащий множество $\mathrm{P}_{k}$, зависит только от $k, \mathcal{E}$ и $\mathcal{E}_{c}$. Иными словами, этот эллипс оказывается одним и тем же при любом выборе траектории в $\mathcal{E}$ с каустикой $\mathcal{E}_{c}$.

ДокАЗАтЕльство. Этот результат доказывается с использованием теоремы о двойном отражении, аналогично теореме 3.48. Однако мы проведем доказательство иначе, что даст возможность явно вычислить эллипс, содержащий $\mathrm{P}_{k}$.

Биллиарду в $\mathcal{E}$ и фиксированной каустике $\mathcal{E}_{c}$ можно сопоставить эллиптическую кривую (см. [15], [16], [30], [52]). Каждой точке этой кривой соответствует коника из софокусного семейства. С другой стороны, биллиардное движение можно рассматривать как линейное движение по якобиану кривой (т. е. по самой кривой, поскольку мы в эллиптическом случае), со сдвигами, соответствующими отскокам. Точнее, сдвиг происходит в точности на элемент эллиптической кривой, отвечающий эллипсу, от которого отражается отрезок траектории.

Итак, если точке $M$ эллиптической кривой сопоставлен эллипс $\mathcal{E}$, то множество $\mathrm{P}_{k}$ лежит на эллипсе, сопоставленном точке $k M$.

3.6.3. Сетки в произвольной размерности. Хотя теорема 3.48 является некоторым продвижением в понимании сеток Понселе-Дарбу, существенным прорывом в этом направлении, с нашей точки зрения, оказывается исследование многомерного случая. Этот анализ основывается на введении многомерных аналогов сеток и введенном нами понятии $s$-скрещенных прямых.

Теорема 3.54. Пусть $\left(a_{m}\right)_{m \in \mathbb{Z}},\left(b_{m}\right)_{m \in \mathbb{Z}}-$ две последователъности отрезков биллиардных траекторий внутри эллипсоида $\mathcal{E}$ в $\mathbb{E}^{d}$, имеющих одну и ту же $d-1$ каустику. Допустим, что пара $\left(a_{0}, b_{0}\right)$ s-скрещена и что последовательность отражений от квадрик $\mathcal{Q}^{1}, \ldots, \mathcal{Q}^{s+1}$ реализует минимальную биллиардную траекторию, связывающую $a_{0} c b_{0}$.

Тогда всякая пара $\left(a_{m}, b_{m}\right)$ s-скрещена и минимальная биллиардная траектория, связывающая эту пару прямых, задается последовательностъю отражений от тех же квадрик $\mathcal{Q}^{1}, \ldots, \mathcal{Q}^{s+1}$.

ДокАЗАТЕльство. Доказательство можно провести с использованием теоремы о двойном отражении аналогично теореме 3.48 .

Этот результат можно сформулировать и для произвольной квадрики, а не только для эллипсоида.

ПреДЛОЖеНИЕ 3.55. Пустъ $\mathcal{E}$ - эллипсоид в $\mathbb{E}^{d}, \mathcal{Q}_{1}, \ldots, \mathcal{Q}_{d-1}$ - собокусные с $\mathcal{E}$ квадрики и $k$ - целое число.

Предположим, что существует траектория $\left(\ell_{m}\right)$ биллиарда внутри $\mathcal{E}$ с каустиками $\mathcal{Q}_{1}, \ldots, \mathcal{Q}_{d-1}$, у которой пара $\left(\ell_{0}, \ell_{k}\right)$ s-скрещена, причем минимальная биллиардная траектория, соединяющая $\ell_{0} c \ell_{k}$, реализуется последовательностъю отражений от квадрик $\mathcal{Q}^{1}, \ldots, \mathcal{Q}^{s+1}$, софокусных с $\mathcal{E}$. 
Тогда для всякой биллиардной траектории $\left(\hat{\ell}_{m}\right)$ внутри $\mathcal{E}$ с каустиками $\mathcal{Q}_{1}, \ldots, \mathcal{Q}_{d-1}$, пары прямых $\left(\hat{\ell}_{m}, \hat{\ell}_{m+k}\right)$ s-скрещены и минимальная биллиардная траектория, соединяющая $\hat{\ell}_{m} c \hat{\ell}_{m+k}$, реализуется последовательностью отражений от $\mathcal{Q}^{1}, \ldots, \mathcal{Q}^{s+1}$.

Авторы признательны рецензенту за полезные замечания.

\section{Список литературы}

[1] В. В. Козлов, Д. В. Трещёв, Биллиарды. Генетическое введение в динамику систем с ударами, Изд-во МГУ, М., 1991, ISBN: 5-211-01566-5, 168 с.; англ. пер.: V.V. Kozlov, D. V. Treschev, Billiards. A genetic introduction to the dynamics of systems with impacts, Transl. Math. Monogr., 89, Amer. Math. Soc., Providence, RI, 1991, ISBN: 0-8218-4550-0, viii+171 pp.

[2] J. V. Poncelet, Traité des propriétés projectives des figures, Mett, Paris, 1822; Reprint of the 2nd (1866) ed.: vol. I, II, Les Grands Classiques Gauthier-Villars, Gauthier-Villars, Sceaux, 1995, vol. I, ISBN: 2-87647-132-9, xxxii+429 pp.; vol. II, viii+453 pp.

[3] Я.К.Г. Якоби, Лекиии по динамике, 2-е изд., стер., УРСС, М., 2004, ISBN: 5-354-00689-9, 271 с.; пер. с нем.: C. G.J. Jacobi, Vorlesungen über Dynamik. Gesammelte Werke, Supplementband, G. Reimer, Berlin, 1884.

[4] Ph. Griffiths, J. Harris, "A Poncelet theorem in space", Comment. Math. Helv., 52:2 (1977), 145-160.

[5] G. Darboux, Principes de géométrie analytique, Gauthier-Villars, Paris, 1917.

[6] N. Trudi, "Rappresentazione geometrica immediata dell' equazione fondamentale nella teoria delle funzioni ellitiche con diverse applicazioni", Memoria della R. Accademia delle Scienze di Napoli, 1853, 63-99.

[7] N. Trudi, "Studii intorno ad una singolare eliminazione, con applicazione alla ricerca delle relazione tra gli elementi di due coniche, l'una iscritta, l'altra circoscritta ad un poligono, ed ai corrispondenti teoremi di Poncelet", Atti di Napoli, 1 (1863).

[8] V. M. Buchstaber, A. P. Veselov, "Integrable correspondences and algebraic representations of multivalued groups", Internat. Math. Res. Notices, 8 (1996), 381-400.

[9] A. Cayley, "Developments on the porism of the in-and-circumscribed polygon", Philosophical magazine, 7 (1854), 339-345.

[10] A. Cayley, "Note on the porism of the in-and-circumscribed polygon", Philosophical magazine, 6 (1853), 99-102.

[11] A. Cayley, "On the porism of the in-and-circumscribed triangle, and on an irrational transformation of two ternary quadratic forms each into itself", Philosophical magazine, 9 (1855), 513-518.

[12] A. Cayley, "On the porism of the in-and-circumscribed triangle", Quart. Math. J., 1 (1857), 344-354.

[13] A. Cayley, "On the a posteriori demonstration of the porism of the in-and-circumscribed triangle", Quart. Math. J., 2 (1858), 31-38.

[14] A. Cayley, "On the porism of the in-and-circumscribed polygon", Philos. Trans. $R$. Soc. Lond., 51 (1861), 225-239.

[15] H. Lebesgue, Les coniques, Gauthier-Villars, Paris, 1942, viii+190 pp.

[16] Ph. Griffiths, J. Harris, "On Cayley's explicit solution to Poncelet's porism", Enseign. Math. (2), 24:1-2 (1978), 31-40.

[17] M. Berger, Geometry, vol. I, II, Universitext, Springer-Verlag, Berlin, 1987, vol. I, ISBN: 3-540-11658-3, xiv+428 pp.; vol. II, x+406 pp.

[18] W. Barth, Th. Bauer, "Poncelet theorems", Expo. Math., 14:2 (1996), 125-144. 
[19] W. Barth, J. Michel, "Modular curves and Poncelet polygons", Math. Ann., 295:1 (1993), 25-49.

[20] B. Jakob, "Moduli of Poncelet polygons", J. Reine Angew. Math., 436 (1993), 33-44.

[21] Shau-Jin Chang, B. Crespi, Kang-Jie Shi, "Elliptical billiard systems and the full Poncelet's theorem in $n$ dimensions", J. Math. Phys., 34:6 (1993), 2242-2256.

[22] В. И. Арнольд, Математические методы классической механики, Учеб. пособие, Наука, M., 1974, 431 с.; англ. пер.: V. I. Arnold, Mathematical methods of classical mechanics, Grad. Texts in Math., 60, Springer-Verlag, New York-Heidelberg, 1978, ISBN: 0-387-90314-3, x+462 pp.

[23] G. Darboux, "Sur les polygones inscrits et circonscrits à l'ellipsoïde", Bulletin de la Société philomathique, 7 (1870), 92-94.

[24] V. Dragović, M. Radnović, "Conditions of Cayley's type for ellipsoidal billiard", J. Math. Phys., 39:1 (1998), 355-362.

[25] V. Dragović, M. Radnović, "On periodical trajectories of the billiard systems within an ellipsoid in $\mathbb{R}^{d}$ and generalized Cayley's condition", J. Math. Phys., 39:11 (1998), 5866-5869.

[26] V. Dragović, M. Radnović, "Cayley-type conditions for billiards within $k$ quadrics in $\mathbb{R}^{d ", ~ J . ~ P h y s . ~ A, ~ 37: 4 ~(2004), ~ 1269-1276 . ~}$

[27] V. Dragović, M. Radnović, "Corrigendum: Cayley-type conditions for billiards within $k$ quadrics in $\mathbb{R}^{d ",}$ J. Phys. A, 38:36 (2005), 7927.

[28] V. Dragović, M. Radnović, "A survey of the analytical description of periodic elliptical billiard trajectories", J. Math. Sci. (N. Y.), 135:4 (2006), 3244-3255.

[29] V. Dragović, M. Radnović, "Geometry of integrable billiards and pencils of quadrics", J. Math. Pures Appl. (9), 85:6 (2006), 758-790.

[30] J. Moser, A.P. Veselov, "Discrete versions of some classical integrable systems and factorization of matrix polynomials", Comm. Math. Phys., 139:2 (1991), 217-243.

[31] A.P. Veselov, "Confocal surfaces and integrable billiards on the sphere and in the Lobachevsky space", J. Geom. Phys., 7:1 (1990), 81-107.

[32] V. Dragović, B. Jovanović, M. Radnović, "On elliptical billiards in the Lobachevsky space and associated geodesic hierarchies", J. Geom. Phys., 47:2-3 (2003), 221-234.

[33] V. Dragović, M. Radnović, "Bifurcations of Liouville tori in elliptical billiards", Regul. Chaotic Dyn., 14:4-5 (2009), 479-494.

[34] V. Dragović, "On integrable potential perturbations of the Jacobi problem for the geodesics on the ellipsoid", J. Phys. A, 29:13 (1996), L317-L321.

[35] В.И. Драгович, "Интегрируемые возмущения биллиарда Биркгофа внутри эллипса", ПММ, 62:1 (1998), 166-168; англ. пер.: V.I. Dragovich, "Integrable perturbations of the Birkhoff billiard inside an ellipse", J. Appl. Math. Mech., 62:1 (1998), 159-162.

[36] V. Dragović, "The Appell hypergeometric functions and classical separable mechanical systems", J. Phys. A, 35:9 (2002), 2213-2221.

[37] Ю.Н. Федоров, "Эллипсоидальный биллиард с квадратичным потенциалом", Функи. анализ и его прил., 35:3 (2001), 48-59; англ. пер.: Y. N. Fedorov, "An ellipsoidal billiard with quadratic potential", Funct. Anal. Appl., 35:3 (2001), 199-208.

[38] В.В. Козлов, "Некоторые интегрируемые обобщения задачи Якоби о геодезических на эллипсоиде", ПММ, 59:1 (1995), 3-9; англ. пер.: V. V. Kozlov, "Some integrable generalizations of the Jacobi problem on geodesics on an ellipsoid", J. Appl. Math. Mech., 59:1 (1995), 1-7.

[39] H. Knörrer, "Geodesics on the ellipsoid", Invent. Math., 59:2 (1980), 119-143.

[40] M. Reid, The complete intersection of two or more quadrics, $\mathrm{Ph}$. D. Thesis, Trinity College, Cambridge, 1972.

[41] V. Dragović, M. Radnović, "Hyperelliptic Jacobians as billiard algebra of pencils of quadrics: Beyond Poncelet porisms", Adv. Math., 219:5 (2008), 1577-1607. 
[42] R. Donagi, "Group law on the intersection of two quadrics", Ann. Scuola Norm. Sup. Pisa Cl. Sci. (4), 7:2 (1980), 217-239.

[43] E. Weyr, "Über einige Sätze von Steiner und ihren Zusammenhang mit der zwei und zweigliedrigen Verwandtschaft der Grundgebilde ersten Grades", J. Reine Angew. Math., 71 (1870), 18-28.

[44] G. Darboux, Leçons sur la théorie générale des surfaces et les applications géométriques du calcul infinitésimal, vol. 2, 3, Gauthier-Villars, Paris, 1915.

[45] В. В. Козлов, "Условия рациональности отношения эллиптических интегралов и большая теорема Понселе", Вест. МГУ. Сер. 1. Матем., мех., 2003, № 4, 6-13; англ. пер.: V. V. Kozlov, "Rationality conditions for the ratio of elliptic integrals and the great Poncelet theorem", Moscow Univ. Math. Bull., 58:4 (2003), 1-7.

[46] V. V. Kozlov, "Billiards, invariant measures, and equilibrium thermodynamics. II", Regul. Chaotic Dyn., 9:2 (2004), 91-100.

[47] В. В. Козлов, "Двузвенные биллиардные траектории: экстремальные свойства и устойчивость", ПМM, 64:6 (2000), 942-946; англ. пер.: V. V. Kozlov, "Two-link billiard trajectories: extremal properties and stability", J. Appl. Math. Mech., 64:6 (2000), 903-907.

[48] V.V. Kozlov, "Billiards, invariant measures, and equilibrium thermodynamics", Regul. Chaotic Dyn., 5:2 (2000), 129-138.

[49] E. Gutkin, S. Tabachnikov, "Billiards in Finsler and Minkowski geometries", J. Geom. Phys., 40:3-4 (2002), 277-301.

[50] M. Radnović, "A note on billiard systems in Finsler plane with elliptic indicatrices", Publ. Inst. Math. (Beograd) (N.S.), 74 (2003), 97-102.

[51] S. Tabachnikov, "Ellipsoids, complete integrability and hyperbolic geometry", Mosc. Math. J., 2:1 (2002), 185-196.

[52] J. Moser, "Geometry of quadrics and spectral theory", The Chern Symposium, 1979: Proceedings of the International Symposium on Differential Geometry in honor of S.-S. Chern (Berkeley, CA, 1979), Springer, New York-Berlin, 1980, 147-188.

[53] Д. Д. Мордухай-Болтовской, "Теорема Понслэ на плоскости Лобачевского и эллиптические интегралы”, Докл. АН СССР, 77:6 (1951), 961-964.

[54] Shau Jin Chang, Kang Jie Shi, "Billiard systems on quadric surfaces and the Poncelet theorem", J. Math. Phys., 30:4 (1989), 798-804.

[55] А.В. Болсинов, А.Т. Фоменко, Интегрируемые гамильтоновы системы. Геометрия, топология, классификаиия, т. 1, 2, РХД, Ижевск, 1999, ISBN: 5-70290352-8, 444 с., 446 с.; англ. пер.: A. V. Bolsinov, A. T. Fomenko, Integrable Hamiltonian systems. Geometry, topology, classification, Chapman \& Hall, Boca Raton, FL, 2004, ISBN: 0-415-29805-9, 730 pp.

[56] А. В. Болсинов, С. В. Матвеев, А. Т. Фоменко, "Топологическая классификация интегрируемых гамильтоновых систем с двумя степенями свободы. Список систем малой сложности", УМH, 45:2 (1990), 49-77; англ. пер.: А. V. Bolsinov, S. V. Matveev, A. T. Fomenko, "Topological classification of integrable Hamiltonian systems with two degrees of freedom. List of systems with small complexity", Russian Math. Surveys, 45:2 (1990), 59-94.

[57] A. V. Bolsinov, A. A. Oshemkov, "Singularities of integrable Hamiltonian systems", Topological methods in the theory of integrable systems, Cambridge Sci. Publ., Cambridge, 2006, 1-67.

[58] E. Shlizerman, V. Rom-Kedar, "Hierarchy of bifurcations in the truncated and forced nonlinear Schrödinger model", Chaos, 15:1 (2005), 013107, 22 pp.

[59] M. Radnović, V. Rom-Kedar, "Foliations of isonergy surfaces and singularities of curves", Regul. Chaotic Dyn., 13:6 (2008), 645-668.

[60] H. Waalkens, H. R. Dullin, "Quantum monodromy in prolate ellipsoidal billiards", Ann. Physics, 295:1 (2002), 81-112. 
[61] С. В. Болотин, "Интегрируемые бильярды Биркгофа", Вестн. Моск. ун-та. Сер. Матем., мех., 1990, № 2, 33-36; англ. пер.: S. V. Bolotin, "Integrable Birkhoff billiards", Mosc. Univ. Mech. Bull., 45:2 (1990), 1-13.

[62] D. Turaev, V. Rom-Kedar, "Soft billiards with corners", J. Statist. Phys., 112:3-4 (2003), 765-813.

[63] A. V. Bolsinov, A. T. Fomenko, "The geodesic flow of an ellipsoid is orbitally equivalent to the Euler integrable case in the dynamics of a rigid body", ДАН, 339:3 (1994), 253-296; англ. пер.: А. V. Bolsinov, A. T. Fomenko, "The geodesic flow of an ellipsoid is orbitally equivalent to the integrable Euler case in the dynamics of a rigid body", Russian Acad. Sci. Dokl. Math., 50:3 (1994), 412-417.

[64] M. Audin, "Courbes algébriques et systèmes intégrables: géodesiques des quadriques", Expo. Math., 12:3 (1994), 193-226.

[65] A. Delshams, Y. Fedorov, R. Ramírez-Ros, "Homoclinic billiard orbits inside symmetrically perturbed ellipsoids", Nonlinearity, 14:5 (2001), 1141-1195.

[66] J. Bertrand, "Mémoire sur les intégrales communes à plusieurs problèmes de Mécanique", Jour. de Math., 17 (1852), 121-174.

[67] G. Darboux, "Sur un problème de mécanique", Archives Néerlandaises (2), 6:2 (1901), 371-376.

[68] Э. Т. Уиттекер, Аналитическал динамика, 2-е изд., испр., УРСС, М., 2004, 500 с.; пер. с англ.: Е. T. Whittaker, A treatise on the analytical dynamics of particles and rigid bodies with an introduction to the problem of three bodies, 3rd ed., Univ. Press, Cambridge, 1927, xiv+456 pp.; Reprint of the 1937 edition: Cambridge Math. Lib., Cambridge Univ. Press, Cambridge, 1988, ISBN: 0-521-35883-3, 456 pp.

[69] A. Paul, "Sur les fonctions hypergéométriques de deux variables et sur des équations linéaires aux dérivées partielles", Comptes Rendus, 90 (1880), 296-298.

[70] P. E. Appell, J. Kampé de Fériet, Fonctions hypergéométriques et hypersphériques. Polynômes d'Hermite, Gauthier-Villars, Paris, 1926, 441 pp.

[71] Н. Я. Виленкин, Специальные функиии и теория представления групп, 2-е изд., M., Наука, 1991, ISBN: 5-02-014541-6, 576 с.; англ. пер. 1-го изд.: N. Ja. Vilenkin, Special functions and the theory of group representations, Transl. Math. Monogr., 22, Providence, RI, Amer. Math. Soc., 1968, x+613 pp.

[72] M.S. Narasimhan, S. Ramanan, "Moduli of vector bundles on compact Riemann surfaces", Ann. of Math. (2), 89:1 (1969), 14-51.

[73] А.Н. Тюрин, "О пересечении квадрик", УМН, 30:6 (1975), 51-99; англ. пер.: A. N. Tyurin, "On intersection of quadrics", Russian Math. Surveys, 30:6 (1975), 51-105.

[74] Ф. Гриффитс, Дж. Харрис, Принципы алгебраической геометрии, т. 1, 2, М., Мир, 1982, 496 с., 366 с.; пер. с англ.: Ph. Griffiths, J. Harris, Principles of algebraic geometry, Pure Appl. Math., Wiley, New York, 1978, ISBN: 0-471-32792-1, 813 pp.

[75] A. Hurwitz, "Üeber unendlich-vieldeutige geometrische Aufgaben, insbesondere über die Schliesungsprobleme", Math. Ann., 15:1 (1879), 8-15.

[76] R. E. Schwartz, "The Poncelet grid", Adv. Geom., 7:2 (2007), 157-175.

[77] M. Levi, S. Tabachnikov, "The Poncelet grid and the billiard in ellipses", Amer. Math. Monthly, 114:10 (2007), 895-908.

В. Драгович (V. Dragović)

Математический институт САНИ, Белград, Сербия;

Mathematical Physics Group,

University of Lisbon, Portugal

E-mail: vladad@mi.sanu.ac.rs

\section{М. Раднович (M. Radnović)}

Математический институт САНИ, Белград, Сербия

E-mail: milena@mi.sanu.ac.rs
Поступила в редакцию 03.02 .2010 